\title{
Theoretical formalism for collective electromagnetic response of discrete metamaterial systems
}

\author{
Stewart D. Jenkins and Janne Ruostekoski \\ School of Mathematics and Centre for Photonic Metamaterials, University of Southampton, Southampton SO17 1BJ, United Kingdom
}

(Received 1 June 2012; revised manuscript received 17 July 2012; published 13 August 2012)

\begin{abstract}
We develop a general formalism to describe the propagation of a near-resonant electromagnetic field in a medium composed of magnetodielectric resonators. As the size and the spatial separation of nanofabricated resonators in a metamaterial array are frequently less than the wavelength, we describe them as discrete scatterers, supporting a single mode of current oscillation represented by a single dynamic variable. We derive a Lagrangian and Hamiltonian formalism for the coupled electromagnetic fields and oscillating currents in the length gauge, obtained by the Power-Zienau-Woolley transformation. The response of each resonator to electromagnetic field is then described by polarization and magnetization densities that, to the lowest order in a multipole expansion, generate electric and magnetic dipole excitations. We derive a closed set of equations for the coherently scattered field and normal mode amplitudes of current oscillations of each resonator both within the rotating wave approximation, in which case the radiative decay rate is much smaller than the resonance frequency, and without such an assumption. The set of equations includes the radiative couplings between a discrete set of resonators mediated by the electromagnetic field, fully incorporating recurrent scattering processes to all orders. By considering an example of a two-dimensional split ring resonator metamaterial array, we show that the system responds cooperatively to near-resonant field, exhibiting collective eigenmodes, resonance frequencies, and radiative linewidths that result from strong radiative interactions between closely spaced resonators.
\end{abstract}

DOI: 10.1103/PhysRevB.86.085116

PACS number(s): 42.25.Bs, 45.20.Jj, 42.50.Ct

\section{INTRODUCTION}

Recent advances in nanofabrication provide a variety of tools for engineering the electromagnetic (EM) response of metamaterials in the radiofrequency, microwave, and optical domains. Metamaterials consist of arrays of artificially constructed magnetodielectric resonators that typically interact strongly with the incident and scattered EM fields. These resonator structures frequently extend over length scales smaller than the wavelength of the EM field with which they interact. For example, a metamaterial might comprise isolated circuit elements, or meta-atoms, embedded in a dielectric host medium. Whereas the EM properties of natural atoms are fixed, modifying the design of artificially constructed meta-atoms can endow them with a wide range of electric and/or magnetic responses. Such control allows one to produce materials with EM properties such as negative index of refraction ${ }^{1-3}$ or negative group velocities. ${ }^{4}$ These materials could conceivably be employed to create perfect lenses ${ }^{5,6}$ and electromagnetic cloaks. $^{7-9}$

The exciting EM phenomena of nanofabricated metamaterials can often depend on the effective bulk properties of the sample. Homogenization theories have met with substantial success in describing these properties. ${ }^{2,10-19}$ Homogenization leads to effective continuum models that strive to treat excitations using averaged polarization and magnetization densities formed by current oscillations within the unit-cell resonators. Analyzing an EM response using uniform medium descriptions, however, can be complicated by the fact that recurrent scattering events in which a photon scatters more than once off the same resonator, produce interactions that can strongly influence a system's EM response. ${ }^{20-30}$ In certain circumstances, the bulk permittivity and permeability can be inferred by analyzing the transmission and reflection properties of a metamaterial with finite thickness, ${ }^{10,11}$ or from the scattering properties of a metamaterial's constituent slabs. ${ }^{15-17}$ But, accurate accounting of strong interactions between a metamaterial's unit cells often requires simplifying assumptions such as the elements being arranged in an infinite lattice. ${ }^{2,11-14}$ The discrete translational symmetry of the infinite lattice can be exploited, e.g., to approximate the local field corrections in a medium of discrete magnetoelectric scatterers. $^{31}$

The discrete nature of metamaterials becomes apparent when the infinite lattice symmetry is broken. The strongly interacting nature of these structures renders them very sensitive to finite size effects ${ }^{32,33}$ and to disorder in the lattice. $^{34,35}$ In systems of discrete resonators, interference of different scattering paths between the elements can result, e.g., in light localization. ${ }^{36,37}$ This effect is analogous to Anderson localization of electrons in solids. Even in regular arrays, strong interactions between resonators can find important applications in metamaterial systems, providing precise control and manipulation of EM fields on a subwavelength scale, e.g., by localizing subdiffraction field hot spots. ${ }^{38,39}$ As another example, a system of interacting resonant wires was used to produce a metalens able to transfer subwavelength features of an evanescent field to propagating waves. ${ }^{40}$ In essence, recurrent scattering events produce strong interactions between meta-atoms that contribute to these effects. As a result, ensembles of interacting resonators exhibit a collective mode of oscillation with discrete resonance frequencies and radiative emission rates. In principle, one can calculate the scattered field profile in a metamaterial by having knowledge of how the material comprising the metaatoms reacts to the EM field. One could then numerically integrate Maxwell's equations with a numerical mesh small enough to resolve the features of each meta-atom. This, however, becomes computationally intractable when the system contains more than a few resonator elements.

In this article, we develop a simplified, computationally efficient formalism that captures the fundamental physical 
properties of a finite metamaterial. In particular, we show how EM mediated interactions can form a cooperative response of the metamaterial's constituent resonators. In this model, each unit-cell element, or metamolecule, of the metamaterial array is formed by combinations of circuit elements acting as resonators that interact with the incident and scattered EM fields. In several metamaterial realizations, a metamolecule may further be divided into separate subelements, e.g., isolated circuit elements that can naturally be considered as the elementary building blocks of the metamaterial sample. We refer to such elementary building blocks as meta-atoms. We assume each meta-atom supports a single mode of current oscillation represented by a single dynamic variable.

The theoretical formalism we introduce describes the collective response of a metamaterial array to an incident EM field. To develop this formalism, we begin with the Lagrangian and Hamiltonian representations for charge and current distributions interacting with EM fields. Analyzing the system in the length gauge, obtained by the Power-ZienauWoolley transformation, ${ }^{41-43}$ we derive coupled equations for the EM fields and resonators. A single resonator interacting with its self-generated fields behaves as an LC circuit in which emission of EM radiation damps the current oscillation. An incident EM field drives each resonator. However, each meta-atom is also driven by fields scattered from all other meta-atoms in the metamaterial array. By integrating out the EM fields, we derive a set of equations for the meta-atom current oscillations which describes the collective response of the array to the incident field. Each eigenmode of this system of equations represents a collective oscillation distributed over the resonators with a particular resonance frequency and radiative decay rate. Some modes are superradiant, with emission rates enhanced by collective interactions. In other modes, EM mediated interactions result in subradiant emission in which radiation repeatedly scattered between resonators remains trapped, slowly leaking away from the metamaterial. As an example, we analyze a 2D array of split ring resonators and examine several of its characteristic collective modes. We find that EM mediated interactions can produce a broad distribution of collective emission rates, and that the width of this distribution is sensitive to the inter-resonator spacing. For example, in a $33 \times 33$ array in which the resonators are separated by half a wavelength of the resonant light, the radiative emission rate can be suppressed by five orders of magnitude. On the other hand, when the spacing is increased to 1.4 wavelengths, the emission rate is only suppressed by a factor of five.

In previous Lagrangian treatments, the interaction between elements of a single metamolecule was accounted for by a phenomenological coupling between meta-atom dynamic variables. ${ }^{44}$ Similar phenomenological coupling between nearest-neighbor resonators can also describe the propagation dynamics of excitations in a one-dimensional chain of metamolecules. ${ }^{45}$ Radiative losses were accounted for by additional dissipative terms. However, important effects such as superradiance or subradiance of collective modes cannot be modeled in this way. By contrast, in our treatment, the interactions between meta-atoms are mediated entirely by the scattered EM fields; the radiation lost through decay of one meta-atom can drive another and vice versa. The resulting collective modes of the system can, therefore, exhibit either subradiant or superradiant decay.

This article is organized as follows. We highlight the main results of the developed formalism for the collective response of a metamaterial sample to EM fields in Sec. II. In Sec. III, we set up our description of the metamaterial. We provide a theoretical description of the system dynamics in Sec. IV where we introduce the Lagrangian and derive the Hamiltonian for the system and the equations of motion for the meta-atoms. We also arrive at expressions for the scattered EM fields that drive the meta-atom dynamics. A derivation of our Lagrangian from that describing arbitrary charged particles interacting with the EM field in the Coulomb gauge is provided in Appendix A, and we elaborate on the derivation of the Hamiltonian in Appendix B. We combine the field and meta-atom dynamics in Sec. $\mathrm{V}$ to arrive at coupled equations of motion between meta-atoms in the rotating wave approximation, in which the meta-atom decay rates are much less than their resonance frequencies. A more general model for collective interactions is provided in Appendix C. In Sec. VI, we apply the theoretical formalism to describe collective modes of oscillation in an array of symmetric split ring resonators. Collective modes of these resonators are connected to the linewidth narrowing ${ }^{46}$ of a transmission resonance observed in Ref. 32. In Sec. VII, we quantize this formalism in the special case that the resonators do not suffer from thermal or ohmic losses. Conclusions follow in Sec. VIII.

\section{KEY RESULTS: COLLECTIVE DYNAMICS ARISING FROM RECURRENT EM SCATTERING}

In this section, we summarize key results presented in this article. Ultimately, we describe the collective dynamics arising from an ensemble of magnetodielectric resonators interacting via a near-resonant EM field. When such resonators are placed close to each other, the system can respond to EM fields cooperatively. In order to provide a computationally efficient description, we consider a metamaterial array composed of a set of $N$ discrete meta-atoms. We assume each metaatom $j(j=1, \ldots, N)$ supports a single mode of current oscillation whose behavior is described by a single dynamic variable $Q_{j}$, with units of charge, and its rate of change $I_{j}=\dot{Q}_{j}$, with units of current. As described in Sec. III, the current oscillation produces a polarization density $\mathbf{P}_{j}(\mathbf{r}, t)$ proportional to $Q_{j}$ and a magnetization density $\mathbf{M}_{j}(\mathbf{r}, t)$ proportional to $I_{j}$. An incident wave with electric field $\mathbf{E}_{\mathrm{in}}(\mathbf{r}, t)$ and magnetic induction field $\mathbf{B}_{\mathrm{in}}(\mathbf{r}, t)$ impinges on the system.

For the coupled set of circuit elements and EM fields, we derive a Lagrangian and Hamiltonian formalism in Sec. IV A. The Lagrangian is expressed in the length gauge, obtained by the Power-Zienau-Woolley transformation. ${ }^{41-43}$ For the dynamical variables $Q_{j}$ and the EM vector potential $\mathbf{A}(\mathbf{r})$, we obtain the corresponding conjugate momenta $\phi_{j}$ [see Eq. (19)] and $-\mathbf{D}(\mathbf{r})$ [see Eq. (21)], respectively. Here, $\mathbf{D} \equiv \epsilon_{0} \mathbf{E}+\mathbf{P}$ denotes the electric displacement field. The joint dynamics of the meta-atom and EM fields are then governed by the 
Hamiltonian

$$
\begin{aligned}
\mathcal{H}= & \mathcal{H}_{\mathrm{EM}}+\frac{1}{2 \epsilon_{0}} \int d^{3} r|\mathbf{P}|^{2}+\sum_{j}\left[\frac{1}{2 l_{j}}\left(\phi_{j}-\Phi_{j}\right)^{2}\right. \\
& \left.-\frac{1}{\epsilon_{0}} \int d^{3} r \mathbf{D}(\mathbf{r}, t) \cdot \mathbf{P}_{j}(\mathbf{r})\right],
\end{aligned}
$$

where $\mathcal{H}_{\mathrm{EM}}$ [see Eq. (32)] is the Hamiltonian for the free EM field and $\Phi_{j}$ [see Eq. (17)] is an effective magnetic flux through the meta-atom. The final term of the Hamiltonian accounts for interactions between electric dipoles distributed in the current oscillations and the electric field, while magnetic interactions are contained in $\left(\phi_{j}-\Phi_{j}\right)^{2}$ and arise in the relationships between $\phi_{j}, \Phi_{j}$, and $I_{j}$.

From the Hamiltonian, we derive a coupled set of equations for the EM fields and the meta-atoms. The incident EM fields drive current oscillations within the meta-atoms, thereby inducing polarization and magnetization densities. In Sec. IV C, we derive and integrate the equations for the total EM fields that are expressed in terms of the incident fields and the fields scattered from the polarization and magnetization densities of the meta-atoms. Specifically, currents in meta-atom $j$, when oscillating at a frequency $\Omega$, produce the monochromatic scattered electric field $\mathbf{E}_{\mathrm{S}, j}$ and magnetic field $\mathbf{H}_{\mathrm{S}, j}$ given by

$$
\begin{aligned}
\mathbf{E}_{\mathrm{S}, j}(\mathbf{r}, \Omega)= & \frac{k^{3}}{4 \pi \epsilon_{0}} \int d^{3} r^{\prime}\left[\mathbf{G}\left(\mathbf{r}-\mathbf{r}^{\prime}, \Omega\right) \cdot \mathbf{P}_{j}\left(\mathbf{r}^{\prime}, \Omega\right)\right. \\
& \left.+\frac{1}{c} \mathbf{G}_{\times}\left(\mathbf{r}-\mathbf{r}^{\prime}, \Omega\right) \cdot \mathbf{M}_{j}\left(\mathbf{r}^{\prime}, \Omega\right)\right], \\
\mathbf{H}_{\mathrm{S}, j}(\mathbf{r}, \Omega)= & \frac{k^{3}}{4 \pi} \int d^{3} r^{\prime}\left[\mathbf{G}\left(\mathbf{r}-\mathbf{r}^{\prime}, \Omega\right) \cdot \mathbf{M}_{j}\left(\mathbf{r}^{\prime}, \Omega\right)\right. \\
& \left.-c \mathbf{G}_{\times}\left(\mathbf{r}-\mathbf{r}^{\prime}, \Omega\right) \cdot \mathbf{P}_{j}\left(\mathbf{r}^{\prime}, \Omega\right)\right],
\end{aligned}
$$

where $\mathbf{G}\left(\mathbf{r}-\mathbf{r}^{\prime}, \Omega\right)$ is the radiation kernel connecting an oscillating electric (magnetic) dipole at position $\mathbf{r}^{\prime}$ to the electric (magnetic) field at position $\mathbf{r}$, while $\mathbf{G}_{\times}\left(\mathbf{r}-\mathbf{r}^{\prime}, \Omega\right.$ ) connects an electric (magnetic) dipole at $\mathbf{r}^{\prime}$ to its radiated magnetic (electric) field at $\mathbf{r} .{ }^{47}$ Expressions for these radiation kernels are given in Eqs. (64) and (65).

The total electric and magnetic fields are obtained as a sum of the incident fields and the fields scattered by all the meta-atoms in the system:

$$
\begin{aligned}
& \mathbf{D}(\mathbf{r}, t)=\mathbf{D}_{\text {in }}(\mathbf{r}, t)+\sum_{j} \mathbf{D}_{\mathrm{S}, j}(\mathbf{r}, t), \\
& \mathbf{B}(\mathbf{r}, t)=\mathbf{B}_{\text {in }}(\mathbf{r}, t)+\sum_{j} \mathbf{B}_{\mathrm{S}, j}(\mathbf{r}, t),
\end{aligned}
$$

where we have the scattered magnetic induction $\mathbf{B}_{\mathrm{S}, j} \equiv$ $\mu_{0}\left(\mathbf{H}_{\mathbf{S}, j}+\mathbf{M}_{j}\right)$ and the scattered electric displacement $\mathbf{D}_{\mathbf{S}, j} \equiv$ $\epsilon_{0} \mathbf{E}_{\mathrm{S}, j}+\mathbf{P}_{j}$ from the meta-atom $j$.

Although, according to Eq. (2), the polarization and magnetization densities of all the meta-atoms act as source terms that determine the scattered EM fields, there is, in general, no simple way of solving for the polarization $\mathbf{P}_{j}(\mathbf{r}, \Omega)$ and magnetization $\mathbf{M}_{j}(\mathbf{r}, \Omega)$ densities themselves. The equations for near-resonant EM fields and closely spaced resonators are strongly coupled, and the meta-atoms are driven by both the incident fields and fields scattered by all other meta-atoms in the system. This is illustrated by Hamilton's equations of motion for the resonators,

$$
\begin{aligned}
& \dot{Q}_{j}(t)=I_{j}(t), \\
& \dot{\phi}_{j}(t)=\mathcal{E}_{j}(t),
\end{aligned}
$$

where the total electric field induces an effective electromotive force $(\mathrm{EMF}) \mathcal{E}_{j}$ [see Eq. (17)], driving the meta-atoms.

Solving the coupled dynamical equations for the resonators (5) and the EM fields (2) constitute the central results of the paper. We begin in Sec. V A by considering a single meta-atom. A meta-atom not only feels the influence of the incident EM field and the fields scattered from other meta-atoms, but also its self-generated field. We show that interactions between a meta-atom $j$ and its self-generated field produces an effective damped LC circuit for the current oscillations with self-capacitance $C_{j}$, self-inductance $L_{j}$, and resonance frequency $\omega_{j}$. The oscillating electric and magnetic dipoles of the meta-atom scatter EM fields and therefore induce a radiative decay at rates $\Gamma_{\mathrm{E}, j}$ and $\Gamma_{\mathrm{M}, j}$, respectively.

In a metamaterial array of several meta-atoms, we then solve the coupled set of equations (5) and (2) when each meta-atom is also driven by the scattered fields from all the other meta-atoms. This results in multiple scattering events and yields EM field mediated interactions between the meta-atoms. In particular, when the multiple scattering between closely spaced resonators becomes dominant, so that the EM wave is scattered more than once by the same scatterer (this is called recurrent scattering), the system responds to EM fields cooperatively. In order to analyze the eigenmodes of such a system, it is beneficial to introduce the excitation amplitudes of each meta-atom LC circuit in terms of its dynamical coordinates and the canonical momenta. In particular, when the decay rates are much less than the resonance frequencies, the meta-atom dynamics is well described by the slowly varying normal variables

$$
b_{j}(t) \equiv \frac{e^{i \Omega_{0} t}}{\sqrt{2 \omega_{j}}}\left(\frac{Q_{j}(t)}{\sqrt{C_{j}}}+\frac{\phi_{j}(t)}{\sqrt{L_{j}}}\right) .
$$

In terms of the normal variables, we then derive a linear set of equations for the meta-atoms whose interactions are mediated by the EM fields by explicitly integrating out the scattered fields:

$$
\dot{b}_{j}=\sum_{j^{\prime}} \mathcal{C}_{j, j^{\prime}} b_{j^{\prime}}+f_{\mathrm{in}, j}
$$

The expressions for the coupling matrix $\mathcal{C}$ between the meta-atoms and the driving terms by the incident fields $f_{\text {in, } j}$ are derived in Sec. V B. The diagonal elements of $\mathcal{C}$ reflect the resonance frequencies and decay rates of the single meta-atoms in isolation, while the off-diagonal elements arise from scattered electric and magnetic fields interacting with the meta-atom electric and magnetic dipoles. The coupled equations (7) include the recurrent scattering events between the meta-atoms to all orders. We generalize our treatment to account for stronger interactions between meta-atoms, i.e., where interactions mediated by scattered fields are comparable to the effects of the self-generated field, in Appendix C. 
A system of $N$ meta-atoms supports $N$ collective modes of current oscillation, each matched to an eigenvector of the matrix $\mathcal{C}$. Each mode $i$ has its own collective resonance frequency and decay rate given in terms of its eigenvalue $\lambda_{i}$ as

$$
\begin{aligned}
\Omega_{i} & =-\operatorname{Im}\left(\lambda_{i}\right)+\Omega_{0}, \\
\gamma_{i} & =-2 \operatorname{Re}\left(\lambda_{i}\right),
\end{aligned}
$$

respectively. As a result of the interactions, the collective emission rates can be either much less than (subradiant) or much greater than (superradiant) the constituent single meta-atom decay rates. We demonstrate this in Sec. VI where we consider the collective effects on a 2D metamaterial array of symmetric split ring resonators (SRRs), metamolecules possessing reflection symmetry which consist of two concentric circular arcs of equal length. Even in a relative small metamaterial sample of $33 \times 33$ unit-cell resonators for the lattice spacing of a halfwavelength, we find that the spectrum of resonance frequencies exhibits a long tail of strongly subradiant eigenmodes. The most subradiant mode of the system possesses a radiative decay rate of about five orders of magnitude less than that of an isolated meta-atom. This eigenmode exhibits a checkerboard phase-pattern of dominantly electric dipole excitations. We also find that the strong response of the metamaterial sample is very sensitive to the spacing between the resonators. We analyze the spectrum for the lattice spacing of 1.4 wavelength in which case the distribution of the decay rates is considerably narrowed. The most subradiant mode now has a resonance linewidth that is five times narrower than the one of the isolated unit-cell resonator. Finally, we also provide an example how the propagation dynamics of excitations in a metamaterial array can be analyzed using the collective eigenmodes. We find that the lattice spacing, and hence the interactions between the resonators, strongly influence the rate at which excitations spread over the array.

\section{DISCRETE RESONATOR MODEL OF A METAMATERIAL}

To develop the formalism characterizing interactions of magnetodielectric resonators in EM fields, we first provide a detailed description of the metamaterial and the model we use to represent it. We consider an ensemble of metamolecules, unit-cell elements that comprise the metamaterial, driven by an incident EM field. Each metamolecule can be decomposed into some number of meta-atoms, which may correspond, for example, to individual circuit elements. We model our metamaterial as an ensemble of $N$ meta-atoms. The position of the meta-atom $j$ is denoted by $\mathbf{R}_{j}(j=1, \ldots, N)$. An external beam with electric field $\mathbf{E}_{\text {in }}(\mathbf{r}, t)$ and associated magnetic induction $\mathbf{B}_{\text {in }}(\mathbf{r}, t)$ impinges on the ensemble, driving the meta-atoms. We assume the incident field is bandwidth limited with a spectrum centered at angular frequency $\Omega_{0}$, and that the spatial extent of each meta-atom lies well within a carrier wavelength $\lambda=2 \pi c / \Omega_{0}$.

The meta-atoms may be composed of, e.g., metallic circuit elements supporting plasmonic oscillations, allowing charges, and currents to flow internally. The current and charge distributions produce EM fields, which in turn, influence the dynamics of these distributions. As such, each element supports various eigenmodes of current oscillation. ${ }^{48,49}$ For simplicity, we identify a meta-atom $j$ with a single eigenmode of current oscillation whose state can be described by a single dynamic variable $Q_{j}(t)$ with units of charge and whose spatial profile is described by time-independent functions $\mathbf{p}_{j}(\mathbf{r})$ and $\mathbf{w}_{j}(\mathbf{r})$. These mode functions are defined such that the polarization $\mathbf{P}_{j}(\mathbf{r}, t)$ and magnetization $\mathbf{M}_{j}(\mathbf{r}, t)$ densities associated with atom $j$ are

$$
\begin{aligned}
\mathbf{P}_{j}(\mathbf{r}, t) & =Q_{j}(t) \mathbf{p}_{j}(\mathbf{r}), \\
\mathbf{M}_{j}(\mathbf{r}, t) & =I_{j}(t) \mathbf{w}_{j}(\mathbf{r}),
\end{aligned}
$$

where $I_{j}(t) \equiv d Q_{j} / d t$ is the current. The definitions of the polarization and magnetization lead to the expressions of the charge and current densities within each meta-atom:

$$
\begin{aligned}
\rho_{j}(\mathbf{r}, t) & =-Q_{j}(t) \nabla \cdot \mathbf{p}_{j}(\mathbf{r}), \\
\mathbf{j}_{j}(\mathbf{r}, t) & =\left(\mathbf{p}_{j}(\mathbf{r})+\nabla \times \mathbf{w}_{j}(\mathbf{r})\right) I_{j}(t) .
\end{aligned}
$$

The total polarization and magnetization densities of the system are obtained from a sum over the polarization and magnetization densities of every meta-atom $j$, respectively:

$$
\begin{aligned}
\mathbf{P}(\mathbf{r}, t) & =\sum_{j} \mathbf{P}_{j}(\mathbf{r}, t), \\
\mathbf{M}(\mathbf{r}, t) & =\sum_{j} \mathbf{M}_{j}(\mathbf{r}, t) .
\end{aligned}
$$

We choose the mode functions so that they are zero outside the neighborhood of the meta-atom.

We note that, in general, the various parts of a circuit element contain charge and current densities that could behave independently of one and other; they could therefore be represented by separate dynamic variables. These extra degrees of freedom could be described by assigning multiple modes of current oscillation to the element, each with its own dynamic variable and mode functions to describe the corresponding polarization and magnetization densities. The resulting set of mode function dynamic variables could then interact with one and other via the EM fields. In essence, one could view a circuit element as an ensemble of meta-atoms that touch or overlap with one and other. In this work, however, we have assumed that the mode functions have been chosen so that they are eigenmodes of elements, i.e., there is a zero net interaction between the modes in a given circuit element within a metamolecule. We therefore identify a meta-atom with an eigenmode of current oscillation within a circuit element and treat each meta-atom as possessing only a single mode of current oscillation. This is analogous to approximating an atom interacting with the EM field as a two-level atom. In the present work, we will not address how the eigenmodes of current oscillations are calculated. For isolated circuit elements, they could be computed numerically solving Maxwell's equations using actual material parameters. Alternatively, one could obtain the meta-atom resonance properties directly from experimental measurements, or estimate them using geometrical arguments.

\section{SYSTEM DYNAMICS}

In this section, we introduce the Lagrangian and Hamiltonian formalism for a magnetodielectric medium interacting with EM fields, specifically derived for a system consisting 
of circuit elements whose dynamic variables represent eigenmodes of current oscillations. The Lagrangian is expressed in the length gauge, obtained by the Power-Zienau-Woolley transformation. ${ }^{41-43}$ We find that this particular representation of electromagnetism turns out to be especially useful for describing localized, collectively interacting circuit elements. The specific details of the Power-Zienau-Woolley transformation are covered in Appendix A.

From the Lagrangian, we derive the conjugate momenta for the dynamic variables of the meta-atoms and the EM fields, and the Hamiltonian for the system. The dynamics of the model describe charge and current densities of the system interacting with the EM fields. We derive a coupled set of equations for the EM fields and the resonators in which both the electric and magnetic fields drive the meta-atom dynamics. The expressions for the electric and magnetic fields are obtained in terms of the incident fields illuminating the sample and the fields scattered from the polarization and magnetization densities that represent the meta-atoms in the medium.

\section{A. The Lagrangian and Hamiltonian formalism for meta-atoms interacting with EM fields}

We treat the dynamics of the system in the Coulomb gauge beginning with the Lagrangian formalism. It is particularly advantageous to study the EM response in a gauge representation obtained by the Power-Zienau-Woolley transformation. ${ }^{41-43}$ In Appendix A, we show that the Lagrangian in the PowerZienau-Woolley picture ${ }^{50}$ can be written in terms of meta-atom dynamic variables as

$$
\mathcal{L}=\mathcal{K}+\mathcal{L}_{\mathrm{EM}}+V_{\mathrm{Coul}}+\sum_{j}\left[Q_{j}(t) \mathcal{E}_{j}+I_{j}(t) \Phi_{j}\right],
$$

where $\mathcal{K}$ is an effective kinetic energy given by

$$
\mathcal{K}=\sum_{j} \frac{1}{2} l_{j} I_{j}^{2} .
$$

The phenomenological kinetic inductance $l_{j}$ of meta-atom $j$ provides, within the effective single-particle description of the system, an inertia to the current oscillation that would be present in the absence of EM field interactions. This inertia could result, for example, from the effective mass of charge carriers or surface plasmons within the meta-atom. Excitation of a current oscillation displaces charge carriers from their equilibrium configuration producing a charge density $\rho_{j}(\mathbf{r})$ [Eq. (10a)] within meta-atom $j$. The meta-atom charge densities interact via the instantaneous Coulomb interaction

$$
V_{\text {Coul }}=\frac{1}{8 \pi \epsilon_{0}} \sum_{j, j^{\prime}} \int d^{3} r d^{3} r^{\prime} \frac{\rho_{j}(\mathbf{r}) \rho_{j^{\prime}}\left(\mathbf{r}^{\prime}\right)}{\left|\mathbf{r}-\mathbf{r}^{\prime}\right|} .
$$

The current oscillation in meta-atom $j$ interacts with external EM fields via an effective electromotive force (EMF) $\mathcal{E}_{j}$ and an effective magnetic flux $\Phi_{j}(t)$ through that meta-atom:

$$
\begin{aligned}
\mathcal{E}_{j}(t) & \equiv \int d^{3} r \mathbf{E}(\mathbf{r}, t) \cdot \mathbf{p}_{j}(\mathbf{r}), \\
\Phi_{j}(t) & \equiv \int d^{3} r \mathbf{B}(\mathbf{r}, t) \cdot \mathbf{w}_{j}(\mathbf{r}) .
\end{aligned}
$$

The EMF interacts with the charge $Q_{j}$ on the circuit, while the current $I_{j}$ interacts with the magnetic flux. The Lagrangian for the free EM field $\mathcal{L}_{\mathrm{EM}}$ is given in terms of the Coulomb gauge vector potential $\mathbf{A}$ as

$$
\mathcal{L}_{\mathrm{EM}}=\frac{\epsilon_{0}}{2} \int d^{3} r\left(\left|\frac{d \mathbf{A}}{d t}\right|^{2}-c^{2}|\nabla \times \mathbf{A}|^{2}\right) .
$$

The Lagrangian for the free EM field represents the radiative fields that are responsible for the excitations of the meta-atoms.

We now wish to determine the Hamiltonian for the system. We proceed by identifying the conjugate momenta of the dynamic variables. Those for charges are given by

$$
\phi_{j} \equiv \frac{\partial \mathcal{L}}{\partial I_{j}}=l_{j} I_{j}+\Phi_{j} .
$$

Note that in the limit where $l_{j}$ is vanishingly small, the conjugate momentum of the charge is dominated by the flux through the circuit. This is often the case in microwave metamaterials, where the EM interactions dwarf the effects of charge carrier inertia. The vector potential represents a continuous field of dynamic variables that possess a corresponding continuum of conjugate momenta defined as

$$
\Pi(\mathbf{r}, t) \equiv \frac{\partial \mathcal{L}}{\partial \dot{\mathbf{A}}(\mathbf{r}, t)} .
$$

This conjugate momentum will have a contribution from $\mathcal{L}_{\mathrm{EM}}$ and pick up a contribution from the interaction term $\sum_{j} \mathcal{E}_{j} Q_{j}=\int d^{3} r \mathbf{P} \cdot \mathbf{E}$. For a system of neutral meta-atoms, the conjugate momentum of the vector potential is given by ${ }^{50}$

$$
\Pi(\mathbf{r}, t)=-\mathbf{D}(\mathbf{r}, t),
$$

where

$$
\mathbf{D}=\epsilon_{0} \mathbf{E}+\mathbf{P}
$$

is the electric displacement field.

In treating the field dynamics, it is often convenient to express these fields in terms of the normal variables $a_{\mathbf{q}, \lambda}(t)$ describing a plane wave with wave vector $\mathbf{q}$ and transverse polarization $\hat{\mathbf{e}}_{\hat{\mathbf{q}}, \lambda}$. These normal variables are defined such that the electric displacement and magnetic fields are given by

$$
\begin{gathered}
\mathbf{D}(\mathbf{r}, t)=i \int d^{3} q \xi_{q} \sum_{\lambda} \hat{\mathbf{e}}_{\hat{\mathbf{q}}, \lambda} a_{\mathbf{q}, \lambda}(t) e^{i \mathbf{q} \cdot \mathbf{r}}+\text { c.c. }, \\
\mathbf{B}(\mathbf{r}, t)=i \sqrt{\frac{\mu_{0}}{\epsilon_{0}}} \int d^{3} q \xi_{q} \sum_{\lambda}\left(\hat{\mathbf{q}} \times \hat{\mathbf{e}}_{\hat{\mathbf{q}}, \lambda}\right) a_{\mathbf{q}, \lambda}(t) e^{i \mathbf{q} \cdot \mathbf{r}}+\text { c.c. },
\end{gathered}
$$

respectively, where

$$
\xi_{q} \equiv \sqrt{\frac{c q \epsilon_{0}}{2(2 \pi)^{3}}} .
$$

The normal variables for the EM field satisfy the following relations in terms of the Poisson brackets ${ }^{51}$

$$
\left\{a_{\mathbf{q}, \lambda}, a_{\mathbf{q}^{\prime}, \lambda^{\prime}}^{*}\right\}=-i \delta_{\lambda, \lambda^{\prime}} \delta\left(\mathbf{q}-\mathbf{q}^{\prime}\right),
$$

and $\left\{a_{\mathbf{q}, \lambda}, a_{\mathbf{q}^{\prime}, \lambda^{\prime}}\right\}=\left\{a_{\mathbf{q}, \lambda}, Q_{j}\right\}=\left\{a_{\mathbf{q}, \lambda}, \phi_{j}\right\}=0$.

Having obtained the conjugate momenta and normal field variables, one may write the Hamiltonian for the system by 
applying the Legendre transform

$$
\mathcal{H}=\sum_{j} \dot{Q}_{j} \phi_{j}+\int d^{3} r \dot{\mathbf{A}} \cdot \Pi-\mathcal{L} .
$$

It is beneficial to decompose the Hamiltonian $\mathcal{H}=\mathcal{H}_{\mathrm{mm}}+\mathcal{H}_{\mathrm{E}}$ into a component containing contributions from the meta-atom conjugate momenta, $\mathcal{H}_{\mathrm{mm}}$, and a component accounting for electric field interactions and the free EM field, $\mathcal{H}_{\mathrm{E}}$. The former contribution is given explicitly by

$$
\mathcal{H}_{\mathrm{mm}} \equiv \sum_{j}\left(\phi_{j} I_{j}-\Phi_{j} I_{j}\right)-\mathcal{K} .
$$

But, because $\phi_{j}-\Phi_{j}=l I_{j}$ [see Eq. (19)],

$$
\mathcal{H}_{\mathrm{mm}}=\mathcal{K}=\sum_{j} \frac{\left(\phi_{j}-\Phi_{j}\right)^{2}}{2 l_{j}}
$$

reduces to the kinetic energy of the current oscillations. The terms involving the electric field contribution, on the other hand, are given explicitly by

$$
\mathcal{H}_{\mathrm{E}}=-\int d^{3} r(\dot{\mathbf{A}} \cdot \mathbf{D}+\mathbf{E} \cdot \mathbf{P})-V_{\text {Coul }}-\mathcal{L}_{\text {EM }}
$$

It is beneficial to simplify the contribution of Eq. (30). We carry out this simplification in Appendix B. The total system Hamiltonian may thus be written in the the Power-ZienauWoolley picture as ${ }^{50}$

$$
\begin{aligned}
\mathcal{H}= & \mathcal{H}_{\mathrm{EM}}+\frac{1}{2 \epsilon_{0}} \int d^{3} r|\mathbf{P}|^{2} \\
& +\sum_{j}\left[\frac{1}{2 l_{j}}\left(\phi_{j}-\Phi_{j}\right)^{2}-\frac{1}{\epsilon_{0}} \int d^{3} r \mathbf{D}(\mathbf{r}, t) \cdot \mathbf{P}_{j}(\mathbf{r})\right],
\end{aligned}
$$

where the Hamiltonian for the free EM field is

$$
\begin{aligned}
\mathcal{H}_{\mathrm{EM}} & =\frac{\epsilon_{0}}{2} \int d^{3} r\left(\left|\frac{\Pi}{\epsilon_{0}}\right|^{2}+c^{2}|\nabla \times \mathbf{A}|^{2}\right) \\
& =\int d^{3} q \sum_{\lambda} c q a_{\mathbf{q}, \lambda}^{*} a_{\mathbf{q}, \lambda} .
\end{aligned}
$$

To understand the dynamics that will arise from this Hamiltonian, we examine the physical role of each term individually. The interaction between the displacement field and polarization density can be written in terms of the emitter dynamic variables as

$$
-\int d^{3} r \frac{\mathbf{D}(\mathbf{r}, t)}{\epsilon_{0}} \cdot \mathbf{P}_{j}(\mathbf{r}, t)=-\frac{Q_{j}}{\epsilon_{0}} \int d^{3} r \mathbf{D}(\mathbf{r}, t) \cdot \mathbf{p}_{j}(\mathbf{r}) .
$$

This represents an interaction energy between the electric displacement and the spatial distribution of electric dipoles contained in the polarization density. On the other hand, the interaction with the magnetic field becomes apparent when expanding $\mathcal{K}$, which yields

$$
\frac{\left(\phi_{j}-\Phi_{j}\right)^{2}}{2 l_{j}}=\frac{\phi_{j}^{2}}{2 l_{j}}-\frac{\phi_{j}}{l_{j}} \Phi_{j}+\frac{\Phi_{j}^{2}}{2 l_{j}},
$$

The interaction of meta-atom $j$ with the magnetic field arises in the second term. The physical significance of this interaction can be understood by expressing that contribution in terms of the magnetization density [see Eq. (9)] as

$$
-\frac{\phi_{j}}{l_{j}} \Phi_{j}=-\int d^{3} r \mathbf{M}_{j} \cdot \mathbf{B}-\frac{\Phi_{j}^{2}}{l_{j}} .
$$

Equation (35) effectively contains the interaction between the magnetization density and the magnetic field. Additionally, Eq. (35) includes a term proportional to the square of the magnetic flux. This artifact appears because the magnetization density is a function of the meta-atom current $I_{j}$ rather than its conjugate momentum. When this portion of the interaction is written as entirely in terms of the meta-atom conjugate momentum, the term proportional to the square of the flux disappears. The last term of Eq. (34) represents a diamagnetic interaction proportional to the square of the magnetic field flux through a meta-atom. These interactions are analogous to the effective magnetization and diamagnetic interactions found in the Hamiltonian for electrically charged point particles in Ref. 50.

Finally, we examine the local polarization self-energy term appearing in the Hamiltonian [see Eq. (32)]. This can be expressed in terms of the dynamic variables as

$$
\begin{aligned}
& \frac{1}{2 \epsilon_{0}} \int d^{3} r \mathbf{P}(\mathbf{r}, t) \cdot \mathbf{P}(\mathbf{r}, t) \\
& \quad=\sum_{j, j^{\prime}} \frac{Q_{j} Q_{j^{\prime}}}{2 \epsilon_{0}} \int d^{3} r \mathbf{p}_{j}(\mathbf{r}) \cdot \mathbf{p}_{j^{\prime}}(\mathbf{r}) .
\end{aligned}
$$

When the meta-atoms are spatially separated, however, their polarization mode functions do not overlap, i.e., $\mathbf{p}_{j} \cdot \mathbf{p}_{j^{\prime}} \equiv 0$ for $j \neq j^{\prime}$. The presence of $\mathbf{P}(\mathbf{r}, t) \cdot \mathbf{P}(\mathbf{r}, t)$ results only in an interaction of the meta-atom with itself, which manifests itself as

$$
\frac{1}{2 \epsilon_{0}} \int d^{3} r \mathbf{P}(\mathbf{r}, t) \cdot \mathbf{P}(\mathbf{r}, t)=\sum_{j} \frac{Q_{j}^{2}}{2 \epsilon_{0}} \int d^{3} r\left|\mathbf{p}_{j}(\mathbf{r})\right|^{2} .
$$

If the meta-atoms were to overlap, a contact potential proportional to $Q_{j} Q_{j^{\prime}}$ would appear between the overlapping elements $j$ and $j^{\prime}$. In the initial Lagrangian [see Eq. (13)], direct interactions between the meta-atoms appeared via the instantaneous Coulomb interaction. An advantage of the Hamiltonian treatment in the Power-Zienau-Woolley picture is that such interactions do not appear explicitly; other instantaneous, noncausal contributions to the dynamics cancel out those of the Coulomb interaction. This leaves the metaatom dynamic variables to interact exclusively with the vector potential and its conjugate momentum. Any effective interactions between meta-atoms are thus mediated by these field dynamic variables. ${ }^{50}$

\section{B. The meta-atom dynamics}

The meta-atoms' interaction with the EM fields are illustrated by Hamilton's equations of motion describing the current oscillations:

$$
\begin{aligned}
& \dot{Q}_{j}(t)=\left\{Q_{j}, \mathcal{H}\right\}=I_{j}(t)=\frac{\phi_{j}(t)-\Phi_{j}(t)}{l}, \\
& \dot{\phi}_{j}(t)=\left\{\phi_{j}, \mathcal{H}\right\}=\mathcal{E}_{j}(t) .
\end{aligned}
$$


The conjugate momentum $\phi_{j}$ is driven entirely by the EMF $\mathcal{E}_{j}$, while Eq. (38a) is nothing more than a statement that the rate of change of the charge is the current. At first glance, it may appear that the magnetic field does not drive the meta-atoms. However, its effects manifest themselves indirectly through a relationship between the conjugate momentum $\phi_{j}$ and the current $I_{j}$ that will be discussed in Sec. V. Effective interactions between the resonators come about through multiple scattering of the EM field between resonators.

\section{The scattered EM fields}

In the previous section, we derived the equations for the meta-atoms driven by EM fields. In order to find a coupled set of equations for the fields and the resonators, we need to find how the EM fields depend on the state of the meta-atom charges and currents. In this section, we derive integral expressions for the scattered EM fields where the metamaterial medium acts as a source with effective polarization and magnetization densities. The total electric and magnetic fields are then represented as sums of the incident fields and the scattered fields from the medium. The resulting equations for the resonators and the EM fields are strongly coupled: the resonator dynamics are driven by the EM fields and the fields themselves depend on the excited meta-atom current oscillations.

We begin with the equation of motion for the normal field operators,

$$
\begin{aligned}
\frac{d a_{\mathbf{q}, \lambda}}{d t}= & \left\{a_{\mathbf{q}, \lambda}, \mathcal{H}\right\} \\
= & -i c q a_{\mathbf{q}, \lambda}+e^{i c q t} \frac{\xi_{q}}{\epsilon_{0}} \int d^{3} r^{\prime} e^{-i \mathbf{q} \cdot \mathbf{r}^{\prime}} \\
& \times\left[\hat{\mathbf{e}}_{\hat{\mathbf{q}}, \lambda} \cdot \mathbf{P}\left(\mathbf{r}^{\prime}, t\right)+\frac{1}{c}\left(\hat{\mathbf{q}} \times \hat{\mathbf{e}}_{\hat{\mathbf{q}}, \lambda}\right) \cdot \mathbf{M}\left(\mathbf{r}^{\prime}, t\right)\right],
\end{aligned}
$$

where the first term results in the oscillation of the free EM field and in the second term, we find the polarization and magnetization densities arising from meta-atom currents that act as sources for radiation. Upon integrating Eq. (39), one obtains

$$
\begin{aligned}
a_{\mathbf{q}, \lambda}(t)= & e^{-i c q t} a_{\mathbf{q}, \lambda}^{(\mathrm{in})}+\frac{\xi_{q}}{\epsilon_{0}} \int d^{3} r^{\prime} \int_{-\infty}^{t} d t^{\prime} e^{-i c q\left(t-t^{\prime}\right)} e^{-i \mathbf{q} \cdot \mathbf{r}^{\prime}} \\
& \times\left[\hat{\mathbf{e}}_{\hat{\mathbf{q}}, \lambda} \cdot \mathbf{P}\left(\mathbf{r}^{\prime}, t^{\prime}\right)+\frac{1}{c}\left(\hat{\mathbf{q}} \times \hat{\mathbf{e}}_{\hat{\mathbf{q}}, \lambda}\right) \cdot \mathbf{M}\left(\mathbf{r}^{\prime}, t^{\prime}\right)\right],
\end{aligned}
$$

where $a_{\mathbf{q}, \lambda}^{(\mathrm{in})}=\lim _{t_{0} \rightarrow-\infty} e^{i c q t_{0}} a_{\mathbf{q}, \lambda}\left(t_{0}\right)$ is the initial state of the plane-wave normal variable before it interacts with the resonators.

The incident fields $\left(\mathbf{D}_{\text {in }}, \mathbf{B}_{\text {in }}\right)$ and the scattered fields $\left(\mathbf{D}_{\mathrm{S}}, \mathbf{B}_{\mathrm{S}}\right)$ radiated by the meta-atoms comprise the total electric displacement and magnetic induction fields:

$$
\begin{gathered}
\mathbf{D}(\mathbf{r}, t)=\mathbf{D}_{\text {in }}(\mathbf{r}, t)+\mathbf{D}_{\mathrm{S}}(\mathbf{r}, t), \\
\mathbf{B}(\mathbf{r}, t)=\mathbf{B}_{\text {in }}(\mathbf{r}, t)+\mathbf{B}_{\mathrm{S}}(\mathbf{r}, t), \\
\mathbf{D}_{\mathrm{S}}(\mathbf{r}, t)=\sum_{j} \mathbf{D}_{\mathrm{S}, j}(\mathbf{r}, t), \\
\mathbf{B}_{\mathrm{S}}(\mathbf{r}, t)=\sum_{j} \mathbf{B}_{\mathrm{S}, j}(\mathbf{r}, t),
\end{gathered}
$$

where $\mathbf{D}_{\mathrm{S}, j}(\mathbf{r}, t)$ and $\mathbf{B}_{\mathrm{S}, j}(\mathbf{r}, t)$ denote the fields emitted by the meta-atom $j$. The incident fields $\mathbf{D}_{\text {in }}$ and $\mathbf{B}_{\text {in }}$ are given in the plane-wave representation by Eqs. (23) and (24) with $e^{-i c q t} a_{\mathbf{q}, \lambda}^{\text {(in) }}$ substituted for $a_{\mathbf{q}, \lambda}$. For the field observation point $\mathbf{r}$ located outside the meta-atoms, we have $\mathbf{D}_{\text {in }}(\mathbf{r}, t)=\epsilon_{0} \mathbf{E}_{\text {in }}(\mathbf{r}, t)$ from Eq. (22). A common situation in experiments corresponds to an illumination of a metamaterial sample by a nonfocused, monochromatic beam that can be approximated by a single plane-wave component, with $\left|\mathbf{k}_{\text {in }}\right|=\Omega_{0} / c$,

$$
\begin{gathered}
\mathbf{D}_{\text {in }}(\mathbf{r}, t)=D_{\text {in }} \hat{\mathbf{e}}_{\text {in }} e^{i\left(\mathbf{k}_{\text {in }} \cdot \mathbf{r}-\Omega_{0} t\right)}+\text { c.c. }, \\
\mathbf{B}_{\text {in }}(\mathbf{r}, t)=\sqrt{\frac{\mu_{0}}{\epsilon_{0}}} \hat{\mathbf{k}}_{\text {in }} \times \mathbf{D}_{\text {in }}(\mathbf{r}, t) .
\end{gathered}
$$

One obtains explicit expressions for $\mathbf{D}$ and $\mathbf{B}$, by substituting Eq. (40) into Eqs. (23) and (24), summing over the two transverse polarizations $\hat{\mathbf{e}}_{\hat{\mathbf{q}}, \lambda}$, and integrating over $\mathbf{q}$. Following this procedure, one obtains the scattered fields

$$
\begin{aligned}
\mathbf{D}_{\mathrm{S}}(\mathbf{r}, t)= & \int_{-\infty}^{t} \int d^{3} r^{\prime}\left[\mathbf{S}\left(\mathbf{r}-\mathbf{r}^{\prime}, t-t^{\prime}\right) \cdot \mathbf{P}\left(\mathbf{r}^{\prime}, t^{\prime}\right)\right. \\
& \left.+\frac{1}{c} \mathbf{S}_{\times}\left(\mathbf{r}-\mathbf{r}^{\prime}, t-t^{\prime}\right) \cdot \mathbf{M}\left(\mathbf{r}^{\prime}, t^{\prime}\right)\right]
\end{aligned}
$$

and

$$
\begin{aligned}
\mathbf{B}_{\mathrm{S}}(\mathbf{r}, t)= & \mu_{0} \int_{-\infty}^{t} \int d^{3} r^{\prime}\left[\mathbf{S}\left(\mathbf{r}-\mathbf{r}^{\prime}, t-t^{\prime}\right) \cdot \mathbf{M}\left(\mathbf{r}^{\prime}, t^{\prime}\right)\right. \\
& \left.-c \mathbf{S}_{\times}\left(\mathbf{r}-\mathbf{r}^{\prime}, t-t^{\prime}\right) \cdot \mathbf{P}\left(\mathbf{r}^{\prime}, t^{\prime}\right)\right]
\end{aligned}
$$

where $\mathbf{S}$ is the propagator that takes the electric (magnetic) field from the electric (magnetic) dipole source $\mathbf{r}^{\prime}$ to the observation point at $\mathbf{r}$ and $\mathbf{S}_{\times}$represents the propagation of the radiated electric (magnetic) field from the magnetic (electric) dipole sources to the observation points. The propagator $\mathbf{S}$ is given by

$$
\begin{aligned}
\mathbf{S}(\mathbf{r}, t) & =\frac{i c}{16 \pi^{3}} \int d^{3} q k(\mathbf{1}-\hat{\mathbf{q}} \hat{\mathbf{q}}) e^{i \mathbf{q} \cdot \mathbf{r}}\left(e^{-i c q t}-e^{i c q t}\right) \\
& =\frac{c}{4 \pi}\left(\nabla \nabla-\mathbf{1} \nabla^{2}\right) \frac{\delta(r-c t)-\delta(r+c t)}{r}
\end{aligned}
$$

The two $\delta$ functions produce retarded and advanced time contributions to the scattered fields, with the advanced time's contribution arising only at $r=0$. The derivatives acting on $1 / r$ result in a contact interaction proportional to $\delta(\mathbf{r})$. At first glance, the retarded and advanced time contributions may appear to cancel out at $r=0$, thus nullifying such a contact interaction. However, by examining the frequency components of $\mathbf{S}$, one can show that this contact interaction does survive. ${ }^{23}$ The corresponding expressions for $\mathbf{S}_{\times}$are

$$
\begin{aligned}
\mathbf{S}_{\times}(\mathbf{r}, t) & =-\frac{i c}{16 \pi^{3}} \int d^{3} q e^{i \mathbf{q} \cdot \mathbf{r}}\left(e^{-i c q t}+e^{i c q t}\right) \mathbf{q} \times \mathbf{1} \\
& =-\nabla \times \frac{1}{4 \pi r} \frac{\partial}{\partial t}[\delta(r-c t)-\delta(r+c t)] .
\end{aligned}
$$

From the oscillator equations of motion [see Eq. (38)], we find that the fields emitted from one resonator will drive all of the others. The driven resonators, in turn, rescatter these fields to yet other resonators in the metamaterial. To more easily account for the cumulative effects of these multiple scatterings 
and identify collective modes in the system, we analyze the field and oscillator dynamics in the frequency domain.

We therefore decompose the source fields $\mathbf{P}$ and $\mathbf{M}$ into their frequency components and compute the scattered monochromatic constituents of the EM fields. Specifically, we write an arbitrary source field,

$$
\mathbf{f}(\mathbf{r}, t)=\frac{1}{\sqrt{2 \pi}} \int_{-\infty}^{\infty} d \Omega \mathbf{f}(\mathbf{r}, \Omega) e^{-i \Omega t},
$$

in terms of the Fourier components $\mathbf{f}(\mathbf{r}, \Omega)$. In evaluating the response of the field to each monochromatic source component, one encounters integrals of the form

$$
\begin{gathered}
\int_{-\infty}^{t} d t^{\prime} \mathbf{S}\left(\mathbf{r}-\mathbf{r}^{\prime}, t-t^{\prime}\right) \mathbf{f}\left(\mathbf{r}^{\prime}, \Omega\right) e^{-i \Omega t^{\prime}} \\
=e^{-i \Omega t} \tilde{\mathbf{S}}\left(\mathbf{r}-\mathbf{r}^{\prime}, \Omega\right) \mathbf{f}\left(\mathbf{r}^{\prime}, \Omega\right)
\end{gathered}
$$

and

$$
\begin{gathered}
\int_{-\infty}^{t} d t^{\prime} \mathbf{S}_{\times}\left(\mathbf{r}-\mathbf{r}^{\prime}, t-t^{\prime}\right) \mathbf{f}\left(\mathbf{r}^{\prime}, \Omega\right) e^{-i \Omega t^{\prime}} \\
=e^{-i \Omega t} \tilde{\mathbf{S}}_{\times}\left(\mathbf{r}-\mathbf{r}^{\prime}, \Omega\right) \mathbf{f}\left(\mathbf{r}^{\prime}, \Omega\right)
\end{gathered}
$$

where $\tilde{\mathbf{S}}(\mathbf{r}, \Omega)$ and $\tilde{\mathbf{S}}_{\times}(\mathbf{r}, \Omega)$ are the monochromatic versions of the expressions given in Eqs. (49) and (50) that describe the propagation of the radiated fields from the source to an observation point. In evaluating these propagators, we find it convenient to treat positive and negative frequencies $\Omega$ separately. We, therefore, decompose the propagators as

$$
\begin{gathered}
\tilde{\mathbf{S}}(\mathbf{r}, \Omega)=\tilde{\mathbf{S}}^{(+)}(\mathbf{r}, \Omega) \Theta(\Omega)+\tilde{\mathbf{S}}^{(-)}(\mathbf{r}, \Omega) \Theta(-\Omega), \\
\tilde{\mathbf{S}}_{\times}(\mathbf{r}, \Omega)=\tilde{\mathbf{S}}_{\times}^{(+)}(\mathbf{r}, \Omega) \Theta(\Omega)+\tilde{\mathbf{S}}_{\times}^{(-)}(\mathbf{r}, \Omega) \Theta(-\Omega),
\end{gathered}
$$

where $\Theta$ is the Heaviside function and the propagators' positive and negative frequency components are given by

$$
\tilde{\mathbf{S}}^{( \pm)}(\mathbf{r}, \Omega)=\frac{1}{4 \pi}\left(\nabla \nabla-\mathbf{1} \nabla^{2}\right) \frac{e^{ \pm i k r}}{r}
$$

and

$$
\tilde{\mathbf{S}}_{\times}^{( \pm)}(\mathbf{r}, \Omega)= \pm \frac{i k}{4 \pi}\left(\nabla \times \frac{e^{ \pm i k r}}{r}\right) \mathbf{1},
$$

where $k \equiv|\Omega| / c$ is the angular wave number of the radiation emitted from a monochromatic source of frequency $\Omega$.

One of our goals is to provide radiated electric and magnetic fields $\mathbf{E}$ and $\mathbf{H}$, respectively. These are related to the electric displacement $\mathbf{D}$ and magnetic induction $\mathbf{B}$ by the familiar expressions

$$
\begin{aligned}
\mathbf{E}(\mathbf{r}, t) & =\frac{1}{\epsilon_{0}}[\mathbf{D}(\mathbf{r}, t)-\mathbf{P}(\mathbf{r}, t)], \\
\mathbf{H}(\mathbf{r}, t) & =\frac{1}{\mu_{0}} \mathbf{B}(\mathbf{r}, t)-\mathbf{M}(\mathbf{r}, t) .
\end{aligned}
$$

We thus define dimensionless radiation kernels

$$
\begin{gathered}
\mathbf{G}(\mathbf{r}, \Omega)=\frac{4 \pi}{k^{3}}[\tilde{\mathbf{S}}(\mathbf{r}, \Omega)-\delta(\mathbf{r})], \\
\mathbf{G}_{\times}(\mathbf{r}, \Omega)=\frac{4 \pi}{k^{3}} \tilde{\mathbf{S}}_{\times}(\mathbf{r}, \Omega),
\end{gathered}
$$

where, by the relations of Eqs. (58a) and (58b), the $\delta$ function in Eq. (59) transforms the monochromatic propagators of $\mathbf{D}$ and
$\mathbf{B}$ to those of $\mathbf{E}$ and $\mathbf{H}$, respectively. The Fourier components of the corresponding EM fields are thus given by

$$
\begin{aligned}
\mathbf{E}(\mathbf{r}, \Omega) & =\frac{1}{\epsilon_{0}} \mathbf{D}_{\mathrm{in}}(\mathbf{r}, \Omega)+\sum_{j} \mathbf{E}_{\mathrm{S}, j}(\mathbf{r}, \Omega), \\
\mathbf{H}(\mathbf{r}, \Omega) & =\frac{1}{\mu_{0}} \mathbf{B}_{\mathrm{in}}(\mathbf{r}, \Omega)+\sum_{j} \mathbf{H}_{\mathrm{S}, j}(\mathbf{r}, \Omega),
\end{aligned}
$$

where the fields scattered from meta-atom $j$ are

$$
\begin{aligned}
\mathbf{E}_{\mathrm{S}, j}(\mathbf{r}, \Omega)= & \frac{k^{3}}{4 \pi \epsilon_{0}} \int d^{3} r^{\prime}\left[\mathbf{G}\left(\mathbf{r}-\mathbf{r}^{\prime}, \Omega\right) \cdot \mathbf{P}_{j}\left(\mathbf{r}^{\prime}, \Omega\right)\right. \\
& \left.+\frac{1}{c} \mathbf{G}_{\times}\left(\mathbf{r}-\mathbf{r}^{\prime}, \Omega\right) \cdot \mathbf{M}_{j}\left(\mathbf{r}^{\prime}, \Omega\right)\right], \\
\mathbf{H}_{\mathrm{S}, j}(\mathbf{r}, \Omega)= & \frac{k^{3}}{4 \pi} \int d^{3} r^{\prime}\left[\mathbf{G}\left(\mathbf{r}-\mathbf{r}^{\prime}, \Omega\right) \cdot \mathbf{M}_{j}\left(\mathbf{r}^{\prime}, \Omega\right)\right. \\
& \left.-c \mathbf{G}_{\times}\left(\mathbf{r}-\mathbf{r}^{\prime}, \Omega\right) \cdot \mathbf{P}_{j}\left(\mathbf{r}^{\prime}, \Omega\right)\right] .
\end{aligned}
$$

As with the monochromatic propagators, we decompose the radiation kernels into their positive and negative frequency components as

$$
\begin{aligned}
& \mathbf{G}(\mathbf{r}, \Omega)=\mathbf{G}^{(+)}(\mathbf{r}, \Omega) \Theta(\Omega)+\mathbf{G}^{(-)}(\mathbf{r}, \Omega) \Theta(-\Omega) . \\
& \mathbf{G}(\mathbf{r}, \Omega)=\mathbf{G}_{\times}^{(+)}(\mathbf{r}, \Omega) \Theta(\Omega)+\mathbf{G}_{\times}^{(-)}(\mathbf{r}, \Omega) \Theta(-\Omega) .
\end{aligned}
$$

The radiation kernel $\mathbf{G}^{( \pm)}\left(\mathbf{r}-\mathbf{r}^{\prime}, \Omega\right)$ corresponds to a familiar expression for a radiated electric (magnetic) field at the observation point $\mathbf{r}$, originating from an oscillating electric (magnetic) dipole residing at $\mathbf{r}^{\prime}{ }^{47}$ Similarly, an oscillating electric (magnetic) dipole at $\mathbf{r}^{\prime}$ generates a magnetic (electric) field at $\mathbf{r}$ that is represented by the radiation kernel $\mathbf{G}_{\times}^{( \pm)}(\mathbf{r}-$ $\left.\mathbf{r}^{\prime}, \Omega\right)$. The explicit expressions for these read

$$
\begin{aligned}
& \mathbf{G}^{( \pm)}(\mathbf{r}, \Omega)= i\left[\frac{2}{3} \mathbf{1} h_{0}^{( \pm)}(k r)+\left(\frac{\mathbf{r r}}{r^{2}}-\frac{1}{3} \mathbf{1}\right) h_{2}^{( \pm)}(k r)\right] \\
&-\frac{4 \pi}{3} \mathbf{1} \delta(k \mathbf{r}), \\
& \mathbf{G}_{\times}^{( \pm)}(\mathbf{r}, \Omega)= \pm \frac{i}{k} \nabla \times \frac{e^{ \pm i k r}}{k r} \mathbf{1},
\end{aligned}
$$

where $h_{n}^{(+)}$and $h_{n}^{(-)}$are the spherical Hankel functions with order $n$ of the first and second kinds, respectively, defined by

$$
\begin{aligned}
& h_{0}^{( \pm)}(x)=\mp i \frac{e^{ \pm i x}}{x} \\
& h_{2}^{( \pm)}(x)= \pm i\left(\frac{1}{x} \pm i \frac{3}{x^{2}}-\frac{3}{x^{3}}\right) e^{ \pm i x} .
\end{aligned}
$$

Equations (61)-(65), together with the radiation kernels of Eqs. (66) and (67), constitute the main results of this section. They provide the total electric and magnetic fields both inside and outside the metamaterial sample as a function of polarization and magnetization densities that are produced by oscillating currents in the meta-atoms. Although we have derived the integral expressions for the scattered EM fields in terms of the resonator excitations in Eq. (63), in general, there is no simple way of solving for $\mathbf{P}(\mathbf{r})$ and $\mathbf{M}(\mathbf{r})$. Together with Hamilton's equations for the dynamic variables of the electric charges of the meta-atoms [see Eq. (38)], the formulas for the radiated fields form a coupled set of equations for the EM fields and the matter. The scattered fields from each meta-atom drive 
the dynamics of the other meta-atoms in the system, with the EM fields mediating interactions between the resonators. For the case of near-resonant field excitation and closely spaced circuit elements, the coupling between the EM fields and the meta-atoms can be strong due to multiple scattering processes leading to collective behavior of the system.

In evaluating the scattered fields of Eq. (63), we note that because $h_{2}^{( \pm)}(r)$ contains a $1 / r^{3}$ divergence near $r=0$, the spatial integral of $\mathbf{G}^{( \pm)}(\mathbf{r}, \Omega)$ in Eq. (66) is not absolutely convergent around the origin. However, as Ref. 23 points out, one can handle such a singularity by carving an infinitesimal spherical region around $\mathbf{r}=0$ from the integral and treating this region separately. The integral over the radiation kernel (66) is then defined using the convention that the term inside the brackets vanishes over an infinitesimal volume enclosing the origin. Mathematically, this is achieved by carrying out the integral in this region in spherical coordinates, first integrating over the spherical angles, so that only the $\delta$-function contributes to the integral. (Physically, this indicates an isotropic high-momentum cutoff in the formulation of the nonrelativistic electromagnetism.) With this integration procedure, the $\delta$ function appearing in Eq. (66) is required for the scattered fields to satisfy Gauss' law as well as to produce the correct Maxwell's equations, $\nabla \cdot \mathbf{D}=0$ for a neutral system and $\nabla \cdot \mathbf{B}=0$. The requirement that these conditions are satisfied also confirms that we have duly selected the correct field terms in the Hamiltonian (31) (e.g., electric displacement, instead of electric field) and that the integration procedure of the contact terms [see Eq. (49)] has been performed correctly. While the $\delta$-function singularity in $\mathbf{G}$ does not play a role in the interactions between nonoverlapping meta-atoms, we find in Sec. V A that it does contribute to interactions of a meta-atom with its self-generated field.

The EM fields derived from the Hamiltonian are indeed consistent with Maxwell's equations. To verify this, we check that the positive and negative frequency components of a monochromatic field with wave number $k$ satisfy the wave equations with sources $\mathbf{P}^{( \pm)}$and $\mathbf{M}^{( \pm)}$: ${ }^{47}$

$$
\begin{aligned}
& \left(\nabla^{2}+k^{2}\right) \mathbf{D}^{( \pm)}=-\nabla \times\left(\nabla \times \mathbf{P}^{( \pm)}\right) \mp i \frac{k}{c} \nabla \times \mathbf{M}^{( \pm)}, \\
& \left(\nabla^{2}+k^{2}\right) \mathbf{B}^{( \pm)}=-\mu_{0} \nabla \times\left(\nabla \times \mathbf{M}^{( \pm)}\right) \pm i \mu_{0} c k \nabla \times \mathbf{P}^{( \pm)} .
\end{aligned}
$$

We confirm that the total fields produced by our system satisfy Eqs. (69) and (70) by applying the operator $\left(\nabla^{2}+k^{2}\right)$ to the total electric and magnetic fields [see Eqs. (61) and (62)]. Because the incident waves are composed of superpositions of plane waves, the action of the operator $\left(\nabla^{2}+k^{2}\right)$ on these fields trivially reduces to

$$
\left(\nabla^{2}+k^{2}\right) \mathbf{D}_{\text {in }}=\left(\nabla^{2}+k^{2}\right) \mathbf{B}_{\text {in }}=0 .
$$

Therefore, the only contributions to $\left(\nabla^{2}+k^{2}\right) \mathbf{E}$ and $\left(\nabla^{2}+\right.$ $\left.k^{2}\right) \mathbf{H}$ come from the scattered fields $\mathbf{E}_{\mathrm{S}, j}$ and $\mathbf{H}_{\mathrm{S}, j}$ [Eqs. (63a) and (63b)]. These contributions are most readily determined by expressing the tensor components of the radiation kernels in the differential form

$$
\mathbf{G}_{\times}^{( \pm) \mu, \eta}= \pm \frac{i}{k} \epsilon_{\mu \nu \eta} \frac{\partial}{\partial r_{\nu}} \frac{e^{ \pm i k r}}{k r},
$$

$$
\mathbf{G}^{( \pm) \mu, \eta}=\frac{1}{k^{2}} \frac{\partial}{\partial r_{\mu}} \frac{\partial}{\partial r_{\eta}}-\delta_{\mu \eta} \nabla^{2} \frac{e^{ \pm i k r}}{k r}-4 \pi \delta_{\mu \eta} \delta(k \mathbf{r}) .
$$

Because the differential operators involved in the radiation kernels readily commute with $\left(\nabla^{2}+k^{2}\right)$, the expressions for this operator acting on the scattered fields involve contributions of the form

$$
\left(\nabla^{2}+k^{2}\right) \frac{e^{ \pm i k\left|\mathbf{r}-\mathbf{r}^{\prime}\right|}}{\left|\mathbf{r}-\mathbf{r}^{\prime}\right|}=-4 \pi \delta\left(\mathbf{r}-\mathbf{r}^{\prime}\right) .
$$

appearing under the integral. Physically, the $\delta$ function represents a point source away from which a monochromatic spherical wave $\left(e^{ \pm i k r} / r\right)$ propagates. The resulting expressions for $\left(\nabla^{2}+k^{2}\right) \mathbf{E}_{\mathrm{S}, j}^{( \pm)}$and $\left(\nabla^{2}+k^{2}\right) \mathbf{H}_{\mathrm{S}, j}^{( \pm)}$thus contain integrals over $\delta$ functions, which are readily evaluated. Explicitly, for the component $\mu$ of the scattered electric field, we have

$$
\begin{aligned}
\left(\nabla^{2}+k^{2}\right) D_{\mathrm{S}, j}^{( \pm) \mu}= & -\left(\frac{\partial}{\partial r_{\mu}} \frac{\partial}{\partial r_{\eta}}-\delta_{\mu, \eta} \nabla^{2}\right) P_{j}^{( \pm) \eta} \\
& \mp i \frac{k}{c} \epsilon_{\mu \nu \eta} \frac{\partial}{\partial r_{\nu}} M_{j}^{( \pm) \eta},
\end{aligned}
$$

where

$$
D_{\mathrm{S}, j}^{( \pm) \mu} \equiv \epsilon_{0} E_{\mathrm{S}, j}^{( \pm) \mu}+P_{j}^{( \pm) \mu}
$$

is the $\mu$ th component of the scattered displacement field from meta-atom $j$. Adding the contributions of Eq. (75) for all meta-atoms $j$, produces the equivalent of the wave equation [see Eq. (69)], which is the desired result. Similarly, one finds that by adding the contributions $\sum_{j}\left(\nabla^{2}+k^{2}\right) H_{\mathrm{S}, j}^{( \pm) \mu}$ for all meta-atoms, one recovers the wave equation for the magnetic field [see Eq. (70)].

\section{META-ATOM INTERACTIONS MEDIATED BY THE EM FIELD}

In the previous section, we established how current oscillations in the meta-atoms respond to the EM field [see Eqs. (38)]. Additionally, we arrived at expressions for the electric and magnetic fields scattered by the meta-atoms [see Eqs. (61)-(65)]. These fields were solved in terms of the magnetization and the polarization densities, generated by the resonator excitations. The current oscillations in each meta-atom thus depend on the excitation of all other metaatoms via the scattered radiation. In this section, we combine the response of the meta-atoms to EM field and the expressions of the EM fields scattered by the meta-atoms in order to investigate how the radiation mediates interactions between the meta-atoms.

We begin by examining the dynamics of a single driven meta-atom in Sec. V A. There, we show that when radiative losses are much weaker than the resonance frequency, a single meta-atom's dynamics reduce to those of the familiar damped LC circuit in which the energy is lost to the scattered EM field. We then examine interactions between different meta-atoms in a collection of closely spaced resonators. Due to the strong coupling between the EM fields and the current oscillations, the emitted radiation leads to the collective dynamics of the ensemble. In Sec. V B, we explore the collective response of the system in the rotating wave 
approximation, in which each meta-atom's radiative emission rate is much less than its resonance frequency. We present an analysis for a more strongly interacting system outside the rotating wave approximation in Appendix C.

In these treatments, we assume the spatial extent of each meta-atom is much smaller than the wavelength of EM field with which it interacts. As such, the radiation scattered from each meta-atom can often be approximated as that of electric and magnetic dipoles oscillating in sync with one and other. For simplicity, when evaluating the interactions between metaatoms, we assume that the electric quadrupole and higher order multipole contributions to the radiation of a single meta-atom are much weaker than the dipole radiation and that they can be neglected. This is by no means a necessary approximation. We could extend the general formalism to incorporate multipolefield radiation components in a multipole expansion. The dipole approximation, however, will provide an advantage in maintaining the tractability of the derivation of the collective metamaterial response to EM fields. Moreover, in several practical situations, a unit-cell resonator of a metamaterial array may consist of two or more meta-atoms. Hence, in the dipole approximation to a single meta-atom, the unit-cell resonator would still exhibit multipole radiation contributions. The multipole fields radiated by unit-cell resonators are also weak in many cases. For instance, metamaterial samples consisting of asymmetric split ring metamolecules have been experimentally employed in the studies of collective resonator response. ${ }^{32,34,35,52}$ In an asymmetric split ring metamolecule, the generated quadrupole field is notably suppressed when compared to the corresponding dipolar field. ${ }^{53}$

The electric and magnetic dipole moments produced by the current oscillation in meta-atom $j$ are

$$
\mathbf{d}_{j}=Q_{j} h_{j} \hat{\mathbf{d}}_{j} \quad \text { and } \quad \mathbf{m}_{j}=I_{j} A_{j} \hat{\mathbf{m}}_{j},
$$

respectively. These are given in terms of the charge $Q_{j}$ and the current $I_{j}$ of the meta-atom. The geometry-dependent proportionality coefficients $h_{j}$ and $A_{j}$ have units of length and area and are defined such that

$$
h_{j} \hat{\mathbf{d}}_{j}=\int d^{3} r \mathbf{p}_{j}(\mathbf{r}) \quad \text { and } \quad A_{j} \hat{\mathbf{m}}_{j}=\int d^{3} r \mathbf{w}_{j}(\mathbf{r}) .
$$

The unit vectors $\hat{\mathbf{d}}_{j}$ indicate the orientation of the electric dipole while the unit vectors $\hat{\mathbf{m}}_{j}$ indicate the orientations of the magnetic dipoles. The distributions $\mathbf{p}_{j}(\mathbf{r})$ and $\mathbf{w}_{j}(\mathbf{r})$ [see Eq. (9)] represent the spatial profile of the polarization and magnetization densities in terms of $Q_{j}$ and $I_{j}$. While, generally, the current resulting from the polarization density [the first term in Eq. (10b)] contributes to the magnetic dipole, the polarization and magnetization densities (and hence the mode functions) that lead to a given charge and current distribution are not unique. ${ }^{47}$ We have therefore chosen for each meta-atom $j, \mathbf{p}_{j}, \mathbf{w}_{\mathbf{j}}$, and the position vector $\mathbf{R}_{j}$ such that the contribution of the polarization current to the magnetic dipole moment about $\mathbf{R}_{j}$ is zero.

To facilitate an understanding of how the EM field influences the meta-atom dynamics, we consider a meta-atom's self-generated fields separately from the fields generated externally. Consider the dynamics of a single meta-atom $j$ interacting with the EM field. The meta-atom's equations of motion are given by Eq. (38). To isolate the dynamics arising from the self-generated field, we decompose the electric and magnetic fields into those generated by meta-atom $j-\mathbf{E}_{\mathrm{S}, j}$ and $\mathbf{B}_{\mathrm{S}, j}$-and those generated externally to meta-atom $j$, $\mathbf{E}_{j, \text { ext }}$ and $\mathbf{B}_{j, \text { ext }}$. We then obtain the following relationship between the different contributions:

$$
\begin{aligned}
& \mathbf{E}_{j, \mathrm{ext}} \equiv \mathbf{E}_{\mathrm{in}}+\sum_{j^{\prime} \neq j} \mathbf{E}_{\mathrm{S}, j^{\prime}}, \\
& \mathbf{B}_{j, \mathrm{ext}} \equiv \mathbf{B}_{\mathrm{in}}+\sum_{j^{\prime} \neq j} \mathbf{B}_{\mathrm{S}, j^{\prime}} .
\end{aligned}
$$

These external fields include contributions from the incident field and the fields scattered by all the other meta-atoms in the system.

In the previous section, we derived the expressions for the scattered fields in terms of the polarization and the magnetization densities of the source medium. It was advantageous to represent the scattered fields in the frequency domain. We similarly analyze here the Fourier components of the dynamic variable $Q_{j}$ oscillating at frequency $\Omega$. As we did with the emitted fields, we find it convenient to decompose the meta-atom variables

$$
\begin{gathered}
Q_{j}(t)=Q_{j}^{(+)}(t)+Q_{j}^{(-)}(t), \\
\phi_{j}(t)=\phi_{j}^{(+)}(t)+\phi_{j}^{(-)}(t)
\end{gathered}
$$

into their positive and negative frequency components, with

$$
Q_{j}^{(-)}(t)=\left[Q_{j}^{(+)}(t)\right]^{*}, \quad \phi_{j}^{(-)}(t)=\left[\phi_{j}^{(+)}(t)\right]^{*} .
$$

The positive and negative frequency components for these variables are defined such that, for a given frequency $\Omega$,

$$
\begin{aligned}
& Q_{j}^{( \pm)}(\Omega) \equiv Q_{j}(\Omega) \Theta( \pm \Omega), \\
& \phi_{j}^{( \pm)}(\Omega) \equiv \phi_{j}(\Omega) \Theta( \pm \Omega) .
\end{aligned}
$$

With frequency components of $Q_{j}$ and $\phi_{j}$ defined in this way, the positive (negative) frequency components of the dynamic variables are driven exclusively by the positive (negative) frequency components of the EM fields. Since the metamaterial system we consider in this model is linear, the equations of motion in Fourier space become the algebraic relationships between Fourier components of a common frequency $\Omega$,

$$
\begin{aligned}
-i \Omega Q_{j}(\Omega) & =\frac{\phi_{j}(\Omega)-\Phi_{j, \mathrm{self}}(\Omega)-\Phi_{j, \mathrm{ext}}(\Omega)}{l_{j}}, \\
-i \Omega \phi_{j}(\Omega) & =\mathcal{E}_{j, \mathrm{self}}(\Omega)+\mathcal{E}_{j, \mathrm{ext}}(\Omega),
\end{aligned}
$$

where $\mathcal{E}_{j, \text { self }}$ and $\Phi_{j, \text { self }}$ are the self-generated EMF and flux, respectively, while $\mathcal{E}_{j, \text { ext }}$ and $\Phi_{j \text {,ext }}$ are the EMF and flux generated externally to meta-atom $j$. The current relates to conjugate momentum and magnetic flux through Eq. (19), and the equation of motion for $Q_{j}$, Eq. (84a), is nothing more that the statement that the rate of change of $Q_{j}$ is the current $I_{j}$. This translates to the relationship between frequency components $-i \Omega Q_{j}(\Omega)=I_{j}(\Omega)$. The EMF and magnetic flux contain the external driving induced by the external EM fields as well as driving induced by the field that the current oscillation itself generates. 
The externally applied EMF and magnetic flux are given explicitly in terms of the externally generated fields as

$$
\begin{aligned}
\mathcal{E}_{j, \mathrm{ext}}(\Omega) & \equiv \int d^{3} r \mathbf{p}_{j}(\mathbf{r}) \cdot \mathbf{E}_{j, \mathrm{ext}}(\mathbf{r}, \Omega), \\
\Phi_{j, \mathrm{ext}}(\Omega) & \equiv \int d^{3} r \mathbf{w}_{j}(\mathbf{r}) \cdot \mathbf{B}_{j, \mathrm{ext}}(\mathbf{r}, \Omega) .
\end{aligned}
$$

When the external fields vary slowly over the volume of metaatom $j, \mathcal{E}_{j, \text { ext }}$ and $\Phi_{j \text {,ext }}$ reduce to a direct driving of the metaatoms' electric and magnetic dipoles, respectively:

$$
\begin{aligned}
& \mathcal{E}_{j, \mathrm{ext}}(\Omega) \approx h_{j} \hat{\mathbf{d}}_{j} \cdot \mathbf{E}_{j, \mathrm{ext}}\left(\mathbf{R}_{j}, \Omega\right), \\
& \Phi_{j, \mathrm{ext}}(\Omega) \approx A_{j} \hat{\mathbf{m}}_{j} \cdot \mathbf{B}_{j, \mathrm{ext}}\left(\mathbf{R}_{j}, \Omega\right),
\end{aligned}
$$

where $\mathbf{R}_{j}$ is the position of the meta-atom. The external EMF and flux mediate the interactions between distinct meta-atoms, which we will discuss in Sec. V B and Appendix C.

\section{A. A single meta-atom interacting with the EM field}

Before investigating how scattered EM fields facilitate interactions between meta-atoms, we first shed light on how the meta-atom's field influences the evolution of the meta-atom itself. This is done by studying a single, isolated externally driven meta-atom. We will present expressions for the selfgenerated fields' contribution to both the EMF and the flux. When the spatial extent of the meta-atom is much less than a wavelength, the self-induced EMF can be written in terms of an effective self-capacitance, and the magnetic flux can be written in terms of a magnetic self-inductance. We thus show how each meta-atom can be treated as a radiatively damped LC circuit that is driven by external fields. This analogy allows us to define slowly varying normal variables and derive their dynamics.

\section{Self-induced EMF and magnetic flux}

The EMF and the magnetic flux represent reactions of a meta-atom to EM fields generated by the meta-atom itself as well as to external fields. Self-generated electric and magnetic fields provide a major contribution to the EMF and magnetic flux, respectively. We define the self-generated EMF and flux as

$$
\begin{gathered}
\mathcal{E}_{j, \text { self }}(\Omega) \equiv \int d^{3} r_{j} \mathbf{p}_{j}\left(\mathbf{r}_{j}\right) \cdot \mathbf{E}_{\mathrm{S}, j}\left(\mathbf{r}_{j}, \Omega\right), \\
\Phi_{j, \text { self }}(\Omega) \equiv \int d^{3} r \mathbf{w}_{j}(\mathbf{r}) \cdot \mathbf{B}_{\mathrm{S}, j}(\mathbf{r}, \Omega) .
\end{gathered}
$$

The self-generated fields of meta-atom $j$, i.e., the fields $\mathbf{E}_{\mathrm{S}, j}$ and $\mathbf{H}_{\mathrm{S}, j} \equiv \mathbf{B}_{\mathrm{S}, j} / \mu_{0}-\mathbf{M}_{j}$ scattered from meta-atom $j$, at a frequency $\Omega$ are given in [see Eq. (63)]. From the expression for $\mathbf{E}_{\mathrm{S}, j}$ [see Eq. (63a)], we obtain the self-induced EMF [see Eq. (88)] in terms of the radiation kernels [see Eqs. (66) and (67)]:

$$
\begin{aligned}
\mathcal{E}_{j, \text { self }}(\Omega)= & \frac{k^{3}}{4 \pi \epsilon_{0}} \int d^{3} r \int d^{3} r^{\prime} \\
& \times\left[\mathbf{p}_{j}(\mathbf{r}) \cdot \mathbf{G}\left(\mathbf{r}-\mathbf{r}^{\prime}, \Omega\right) \cdot \mathbf{p}_{j}\left(\mathbf{r}^{\prime}\right) Q_{j}(\Omega)\right. \\
& \left.+\frac{1}{c} \mathbf{p}_{j}(\mathbf{r}) \cdot \mathbf{G}_{\times}\left(\mathbf{r}-\mathbf{r}^{\prime}, \Omega\right) \cdot \mathbf{w}_{j}\left(\mathbf{r}^{\prime}\right) I(\Omega)\right] .
\end{aligned}
$$

Similarly, the self-generated flux is obtained from the expression for $\mathbf{H}_{\mathrm{S}, j}$ [see Eq. (63b)], and is given in terms of the radiation kernels as

$$
\begin{aligned}
\Phi_{j, \text { self }}(\Omega)= & \frac{\mu_{0} k^{3}}{4 \pi} \int d^{3} r\left\{\left|\mathbf{w}_{j}(\mathbf{r})\right|^{2}\right. \\
& +\int d^{3} r^{\prime}\left[\mathbf{w}_{j}(\mathbf{r}) \cdot \mathbf{G}\left(\mathbf{r}-\mathbf{r}^{\prime}, \Omega\right) \cdot \mathbf{w}_{j}\left(\mathbf{r}^{\prime}\right) I_{j}(\Omega)\right. \\
& \left.\left.-c \mathbf{w}_{j}(\mathbf{r}) \cdot \mathbf{G}_{\times}\left(\mathbf{r}-\mathbf{r}^{\prime}, \Omega\right) \cdot \mathbf{p}_{j}\left(\mathbf{r}^{\prime}\right) Q_{j}(\Omega)\right]\right\} .
\end{aligned}
$$

The first term of Eq. (91) arises because the flux is defined in terms of $\mathbf{B}=\mu_{0}(\mathbf{H}+\mathbf{M})$ rather than $\mathbf{H}$, whose scattered field components are determined by the radiation kernels. This results in different contact terms in Eqs. (90) and (91).

Because we have assumed that the meta-atoms are much smaller than the wavelength, we may expand the radiation kernels to lowest order in $k r$ and thus approximate the self-interactions in the near field limit. Since, in this limit, $\mathbf{G}_{\times}(\mathbf{r}, \Omega) / \mathbf{G}(\mathbf{r}, \Omega) \sim k r \ll 1$, we neglect the contribution of $\mathbf{G}_{\times}$to the self-interaction. To leading order in $k r$, we have the positive and negative frequency components of the radiation kernels:

$$
\begin{gathered}
\operatorname{Re}^{( \pm)}(\mathbf{r}, \Omega) \approx \frac{3 \hat{\mathbf{r}} \hat{\mathbf{r}}-\mathbf{1}}{k^{3} r^{3}}-\frac{4 \pi}{3 k^{3}} \delta(\mathbf{r}), \\
\operatorname{Im} \mathbf{G}^{( \pm)}(\mathbf{r}, \Omega) \approx \pm \frac{2}{3}
\end{gathered}
$$

\section{Self-capacitance and self-inductance}

The long wavelength approximation allows us to simplify the expressions for the self-generated EMF and flux [see Eqs. (90) and (91)] by neglecting the contributions of $\mathbf{G}_{\times}$. This approximation implies that the self-induced EMF is directly proportional to the charge $Q_{j}$, and that the self-induced magnetic flux is directly proportional to the current $I_{j}$. We can thus draw an analogy between a typical meta-atom and a standard LC circuit where the charge $Q_{j}$ and current $I_{j}$ are related to $\mathcal{E}_{j \text {,self }}$ and $\Phi_{j \text {,self }}$ through an effective capacitance $C_{j}$ and magnetic self-inductance $L_{j}^{(\mathrm{M})}$. From Eqs. (92) and (93), the positive and negative frequency components of the EMF and flux arising from the meta-atom's self-generated field become

$$
\begin{gathered}
\mathcal{E}_{j, \text { self }}^{( \pm)}(\Omega)=-\left(\frac{1}{C_{j}} \mp i \frac{h_{j}^{2} k^{3}}{6 \pi \epsilon_{0}}\right) Q_{j}^{( \pm)}(\Omega), \\
\Phi_{j, \text { self }}^{( \pm)}(\Omega)=\left(L_{j}^{(\mathrm{M})} \pm i \frac{\mu_{0} A_{j}^{2} k^{3}}{6 \pi}\right) I_{j}^{( \pm)}(\Omega) .
\end{gathered}
$$

In addition to the capacitance and inductance the EMF and flux have respective imaginary contributions that, as we shall see later, represent dissipation of the current oscillation due to radiation being emitted away from the meta-atom. The selfcapacitance $C_{j}$ is given by

$$
\begin{aligned}
\frac{1}{C_{j}}= & \int d^{3} r \frac{\left|\mathbf{p}_{j}(\mathbf{r})\right|^{2}}{3 \epsilon_{0}}-\frac{1}{4 \pi \epsilon_{0}} \int d^{3} r \\
& \times \int d^{3} r^{\prime} \frac{3\left(\mathbf{p}_{j}(\mathbf{r}) \cdot \hat{\mathbf{n}}\right)\left(\hat{\mathbf{n}} \cdot \mathbf{p}_{j}\left(\mathbf{r}^{\prime}\right)\right)-\mathbf{p}_{j}\left(\mathbf{r}^{\prime}\right) \cdot \mathbf{p}_{j}(\mathbf{r})}{\left|\mathbf{r}-\mathbf{r}^{\prime}\right|^{3}}
\end{aligned}
$$


with $\hat{\mathbf{n}} \equiv\left(\mathbf{r}-\mathbf{r}^{\prime}\right) /\left|\mathbf{r}-\mathbf{r}^{\prime}\right|$, and the magnetic self-inductance is

$$
\begin{aligned}
L_{j}^{(\mathrm{M})}= & \frac{2 \mu_{0}}{3} \int d^{3} r\left|\mathbf{w}_{j}(\mathbf{r})\right|^{2}+\frac{\mu_{0}}{4 \pi} \int d^{3} r \\
& \times \int d^{3} r^{\prime} \frac{3\left[\mathbf{w}_{j}(\mathbf{r}) \cdot \hat{\mathbf{n}}\right]\left[\hat{\mathbf{n}} \cdot \mathbf{w}_{j}\left(\mathbf{r}^{\prime}\right]\right)-\mathbf{w}_{j}\left(\mathbf{r}^{\prime}\right) \cdot \mathbf{w}_{j}(\mathbf{r})}{\left|\mathbf{r}-\mathbf{r}^{\prime}\right|^{3}},
\end{aligned}
$$

In essence, excitation of the dynamic variable $Q_{j}$ produces a distribution of electric dipoles (polarization density) proportional to the mode function $\mathbf{p}_{j}(\mathbf{r})$. In the long-wavelength approximation, this distribution of dipoles produces a quasistatic electric field in the vicinity of the meta-atom generated by the real part of the radiation kernel ReG [see Eq. (92)]. The current oscillation interacts with itself via the near field electric dipole-dipole interactions, resulting in the effective capacitance $C_{j}$ appearing in the self-induced EMF [see Eq. (94)]. Similarly, a nonzero current $I_{j}$ produces a distribution of magnetic dipoles (magnetization density) proportional to the mode function $\mathbf{w}_{j}$. The current oscillation then interacts with itself via the near field magnetic dipole-dipole interactions, resulting in the magnetic self-inductance $L_{j}^{(\mathrm{M})}$ appearing in the self-induced flux [see Eq. (95)].

Because the self-induced flux [see Eq. (95)] is proportional to $I_{j}$, we find it convenient to express the conjugate momentum $\phi_{j}=l_{j} I_{j}+\Phi_{j}$ [see Eq. (19)] in terms of a total selfinductance

$$
L_{j} \equiv l_{j}+L_{j}^{(\mathrm{M})} .
$$

This self-inductance includes contributions from both the magnetic and the kinetic inductances. When we include contributions from both the self-generated flux [see Eq. (95)] and the external flux [see Eq. (86)], the conjugate momentum for meta-atom $j$ is given in terms of the total self-inductance by

$$
\phi_{j}^{( \pm)}(\Omega)=\left(L_{j} \pm i \frac{\mu_{0} A_{j}^{2} k^{3}}{6 \pi}\right) I_{j}^{( \pm)}(\Omega)+\Phi_{j, \mathrm{ext}}^{( \pm)}(\Omega) .
$$

This relation will be useful in determining the meta-atom equations of motion.

\section{Equations of motion for a meta-atom interacting with its self-generated fields}

Having determined how the self-scattered fields affect the EMF and flux, we now determine a closed set of equations of motion for the meta-atom's dynamic variable and conjugate momentum. The rate of change of the dynamic variable $Q_{j}$ is given by the current $I_{j}$. Solving Eq. (99) for $I_{j}(\Omega)$ thus allows us to determine an equation of motion for $Q_{j}$ in terms of its conjugate momentum and magnetic flux generated by the external field. Further, substituting the EMF from Eq. (94) into Eq. (84) provides the corresponding equation of motion for $\phi_{j}$. Explicitly these equations of motion are given in the frequency domain as

$$
\begin{aligned}
& -i \Omega Q_{j}^{( \pm)}=\left[1 \mp i\left(\frac{c k}{\omega_{j}}\right)^{3} \frac{\Gamma_{\mathrm{M}, j}}{\omega_{j} D_{j}^{( \pm)}}\right] \frac{\phi_{j}^{( \pm)}}{L_{j}}-\frac{\Phi_{j, \mathrm{ext}}^{( \pm)}}{L_{j} D_{j}^{( \pm)}}, \\
& -i \Omega \phi_{j}^{( \pm)}=-\frac{1}{C_{j}}\left[1 \mp i\left(\frac{c k}{\omega_{j}}\right)^{3} \frac{\Gamma_{\mathrm{E}, j}}{\omega_{j}}\right] Q_{j}^{( \pm)}+\mathcal{E}_{j, \mathrm{ext}}^{( \pm)}
\end{aligned}
$$

where, as we demonstrate later,

$$
\omega_{j} \equiv \frac{1}{\sqrt{L_{j} C_{j}}}
$$

is the single meta-atom resonance frequency, $k \equiv|\Omega| / c$ is the wave number of the field frequency component,

$$
\Gamma_{\mathrm{E}, j} \equiv \frac{h_{j}^{2} C_{j} \omega_{j}^{4}}{6 \pi \epsilon_{0} c^{3}}
$$

is the emission rate due to electric dipole radiation,

$$
\Gamma_{\mathrm{M}, j} \equiv \frac{\mu_{0} A_{j}^{2} \omega_{j}^{4}}{6 \pi c^{3} L_{j}}
$$

is the emission rate due to magnetic dipole radiation, and

$$
D_{j}^{( \pm)}(\Omega)=1 \pm i\left(\frac{c k}{\omega_{j}}\right)^{3} \frac{\Gamma_{j, \mathrm{M}}}{\omega_{j}}
$$

arises from the inversion of Eq. (99). The interaction of the meta-atom with its external fields are parameterized by $h_{j}$ and $A_{j}$ [see Eq. (78)] and hence by the radiative emission rates $\Gamma_{\mathrm{E}, j}$ and $\Gamma_{\mathrm{M}, j}$. This is made clear in the point dipole approximation where we have the external EMF and magnetic flux that drive the meta-atom [see Eq. (87)]. From Eqs. (103) and (104), one can infer that, when the meta-atom geometry is altered such that the self-capacitance and self-inductance remain constant, an increased interaction strength of the meta-atom with the external field corresponds to increased radiative emission rates.

\section{A meta-atom as an LC circuit}

If we neglect the radiative damping and consider a metaatom interacting exclusively with its self-generated field, its dynamics are nothing more than those of an LC circuit with resonance frequency $\omega_{j}$, which in the time domain satisfies the equations of motion

$$
\frac{d}{d t}\left(\begin{array}{c}
Q_{j}(t) \\
\phi_{j}(t)
\end{array}\right)=\left(\begin{array}{cc}
0 & L_{j}^{-1} \\
-C_{j}^{-1} & 0
\end{array}\right)\left(\begin{array}{c}
Q_{j}(t) \\
\phi_{j}(t)
\end{array}\right) .
$$

The meta-atom normal mode variables

$$
\beta_{j} \equiv \frac{1}{\sqrt{2 \omega_{j}}}\left(\frac{Q_{j}}{\sqrt{C_{j}}}+i \frac{\phi_{j}}{\sqrt{L_{j}}}\right)
$$

and $\beta_{j}^{*}$ evolve with eigenfrequencies $\omega_{j}$ and $-\omega_{j}$, respectively,

$$
\beta_{j}(t)=\exp \left(-i \omega_{j} t\right) \beta_{j}(0) .
$$

The collective dynamics within the metamaterial, of course, arise from the interaction of each meta-atom with its external field, necessitating the inclusion of radiative losses $\Gamma_{\mathrm{E}, j}, \Gamma_{\mathrm{M}, j}$. But, as we will see later in this section, the presence of radiative 
interactions not only results in energy being carried away from the meta-atom by the radiated field, but also allows the metaatom to be driven by fields scattered from other meta-atoms.

\section{The meta-atom normal oscillator variables}

The variables $\beta_{j}$ represent eigenmodes of a single metaatom in the absence of interactions with the external fields. The presence of these interactions perturbs the single meta-atom dynamics. Since the incident EM field driving the metamaterial oscillates at a central frequency $\Omega_{0}$, it is convenient to analyze the effects of these perturbations using the slowly varying normal oscillator variables

$$
b_{j}(t)=e^{i \Omega_{0} t} \beta_{j}(t)=\frac{e^{i \Omega_{0} t}}{\sqrt{2 \omega_{j}}}\left(\frac{Q_{j}(t)}{\sqrt{C_{j}}}+\frac{\phi_{j}(t)}{\sqrt{L_{j}}}\right) .
$$

The oscillator variables satisfy the Poisson brackets

$$
\begin{aligned}
\{b(t), b(t)\} & =\left\{b_{j}^{*}(t), b_{j^{\prime}}^{*}(t)\right\}=0, \\
\left\{b_{j}(t), b_{j^{\prime}}^{*}(t)\right\} & =-i \delta_{j, j^{\prime}} .
\end{aligned}
$$

One can recover $Q_{j}$ and $\phi_{j}$ by solving the system of equations formed by Eq. (109) and its complex conjugate. This yields

$$
\begin{aligned}
& \frac{Q_{j}(t)}{\sqrt{\omega_{j} C_{j}}}=\frac{1}{\sqrt{2}}\left[e^{-i \Omega_{0} t} b_{j}(t)+e^{i \Omega_{0} t} b_{j}^{*}(t)\right], \\
& \frac{\phi_{j}(t)}{\sqrt{\omega_{j} L_{j}}}=-i \frac{1}{\sqrt{2}}\left[e^{-i \Omega_{0} t} b_{j}(t)-e^{i \Omega_{0} t} b_{j}^{*}(t)\right] .
\end{aligned}
$$

As the incident electric field may consist of a range of frequencies around $\Omega_{0}$ reflecting its variation in time, it is necessary, in general, to examine the frequency components of the oscillator variables and how they are related to those of $Q_{j}$ and $\phi_{j}$. The Fourier components, for $\Omega>0$, of $Q_{j}$ and $\phi_{j}$ are given in terms of the normal variables as

$$
\begin{gathered}
\frac{Q_{j}^{(+)}(\Omega)}{\sqrt{\omega_{j} C_{j}}}=\frac{1}{\sqrt{2}}\left[b_{j}(\delta)+b_{j}^{*}\left(-\delta-2 \Omega_{0}\right)\right], \\
\frac{\phi_{j}^{(+)}(\Omega)}{\sqrt{\omega_{j} L_{j}}}=-i \frac{1}{\sqrt{2}}\left[b_{j}(\delta)-b_{j}^{*}\left(-\delta-2 \Omega_{0}\right)\right],
\end{gathered}
$$

where

$$
\delta \equiv \Omega-\Omega_{0}
$$

The negative frequency components of $Q_{j}$ and $\phi_{j}$ [given in terms of their positive frequency components in the time domain in Eq. (82)], when $\Omega<0$, can be obtained from the relations, $Q_{j}^{(-)}(\Omega)=\left[Q_{j}^{(+)}(-\Omega)\right]^{*}$ and $\phi_{j}^{(-)}(\Omega)=\left[\phi_{j}^{(+)}(-\Omega)\right]^{*}$.

\section{Dynamics in the rotating wave approximation}

Radiative damping and driving of the meta-atom by external fields alter the current oscillation represented by the normal variable $b_{j}$. The interactions leading to these effects are often sufficiently weak that we can regard their influence as a small perturbation. We consider this weak interaction limit here and in Sec. V B where we examine the collective behavior of the meta-atoms comprising a metamaterial. We thus assume that $b_{j}$ varies slowly with respect to the dominant frequency $\Omega_{0}$ and neglect the fast oscillating components, i.e., we set $b_{j}\left(-\delta-2 \Omega_{0}\right)=0$ for $|\delta| \ll \Omega_{0}$. The mode variables $b_{j}$ are then proportional to the slowly varying envelope of the positive frequency components of the dynamic variables $Q_{j}^{(+)}$and their conjugate momenta $\phi_{j}^{(+)}$. Neglecting fast oscillating components of $b_{j}$ is known as the rotating wave approximation (RWA) and is valid in the limit $\Gamma_{\mathrm{E}, j}, \Gamma_{\mathrm{M}, j},\left|\Omega_{0}-\omega_{j}\right|, \delta \Omega \ll \Omega_{0}$, where $\delta \Omega \ll \Omega_{0}$ indicates a narrow bandwidth of the incident field.

In the RWA, the meta-atom driving forces, i.e., the EMF and flux, can be expressed in terms of their slowly varying envelopes $\tilde{\mathcal{E}}_{j}$ and $\tilde{\Phi}_{j}(t)$ defined such that

$$
\begin{aligned}
& \frac{\mathcal{E}_{j}^{(+)}(t)}{\sqrt{\omega_{j} L_{j}}}=e^{-i \Omega_{0} t} \tilde{\mathcal{E}}_{j}(t), \\
& \frac{\Phi_{j}^{(+)}(t)}{\sqrt{\omega_{j} L_{j}}}=e^{-i \Omega_{0} t} \tilde{\Phi}_{j}(t),
\end{aligned}
$$

where the overall factor of $\sqrt{\omega_{j} L_{j}}$ was included for convenience.

The RWA essentially assumes that all the dynamics are dominated by the frequency $\Omega_{0}$. Because the RWA implies $\delta \Omega,\left|\omega_{j}-\Omega_{0}\right| \ll \Omega_{0}$, we can approximate the quantities $\left(\Omega / \omega_{j}\right)^{3}$ appearing in the equations of motion [see Eqs. (100) and $(101)]$ as $\left(\Omega / \omega_{j}\right)^{3} \approx 1$. In these limits, the equations of motion for the frequency components of $Q_{j}$ and $\phi_{j}$ [see Eqs. (100) and (101)] yield the relationship for the normal variables:

$-i \delta b_{j}(\delta)=\left[-i\left(\omega_{j}-\Omega_{0}\right)-\frac{\Gamma_{\mathrm{E}, j}+\Gamma_{\mathrm{M}, j}}{2}\right] b_{j}(\delta)+f_{j, \mathrm{ext}}(\delta)$,

where the detuning of the meta-atom resonance $\omega_{j}$ from the frequency of the driving field $\Omega_{0}$ manifests itself as an oscillation of the normal variable $b_{j}$ at frequency $\omega_{j}-\Omega_{0}$, while electric and magnetic dipole radiation emanating from the meta-atom results in the damping of $b_{j}$ at a rate $\Gamma_{\mathrm{E}, j}+$ $\Gamma_{\mathrm{M}, j}$. The forcing function combines driving of the current oscillation by the external electric field via the EMF and the external magnetic field via the flux, and is given by

$$
f_{j, \mathrm{ext}}(\delta)=i \frac{1}{\sqrt{2}}\left(\tilde{\mathcal{E}}_{j, \mathrm{ext}}(\delta)+i \omega_{j} \tilde{\Phi}_{j, \mathrm{ext}}(\delta)\right) .
$$

\section{The meta-atom as a driven, $R L C$ circuit}

Here, we show that in the RWA, a meta-atom behaves as a damped, driven RLC circuit interacting with the external driving field. A source of loss that is typically present in a meta-atom which we have thus far neglected is the ohmic losses due to resistance to current flow within the meta-atom. We include the effects of this resistance phenomenologically through the addition of the ohmic loss rate $\Gamma_{\mathrm{O}, j}$ to the radiative damping rate. The approximations leading to Eq. (121) are still valid provided that $\Gamma_{\mathrm{O}, j} \ll \Omega_{0}$. The total meta-atom damping becomes

$$
\Gamma_{j} \equiv \Gamma_{\mathrm{E}, j}+\Gamma_{\mathrm{M}, j}+\Gamma_{\mathrm{O}, j}
$$

We obtain equation of motion for $b_{j}$ in the time domain from Eq. (118) by multiplying by $e^{-i \delta t}$ and integrating over the bandwidth of the external field $-\delta \Omega<\delta<\delta \Omega$,

$$
\frac{d b_{j}}{d t}=\left[-i\left(\omega_{j}-\Omega_{0}\right)-\frac{\Gamma_{j}}{2}\right] b_{j}(t)+f_{j, \mathrm{ext}}(t) .
$$


When the incident field is of finite duration, i.e., $\mathbf{E}_{\text {in }}(\mathbf{r}, \pm \infty)=\mathbf{B}_{\text {in }}(\mathbf{r}, \pm \infty)=0, \quad b_{j} \quad$ satisfies Eq. (121) with the initial condition $b_{j}(-\infty)=0$.

The interaction of the meta-atom's current oscillation with its self-generated EM fields causes the current mode to oscillate at the resonance frequency $\omega_{j}$ [see Eq. (102)] analogous to that of an LC circuit. When a meta-atom current oscillation produces net electric and magnetic dipole moments, this oscillation can be driven by external fields as manifested by the term $f_{j, \text { ext }}(t)$ in Eq. (121). Radiative and ohmic losses act as a resistance within the meta-atom, and the external EMF $\tilde{\mathcal{E}}_{j, \text { ext }}$ and $\tilde{\Phi}_{j, \text { ext }}$ provide the driving.

The dynamics of this effective RLC circuit can be derived from the effective Hamiltonian

$$
H_{\mathrm{eff}, j}=H_{\mathrm{LC}, j}+H_{\mathrm{damp}, j}+V_{\mathrm{ext}, j},
$$

where $H_{\mathrm{LC}, j}$ is the effective Hamiltonian for an undamped LC circuit,

$$
H_{\mathrm{LC}, j}=\omega_{j} b_{j}^{*} b_{j}=\frac{\phi_{j}^{2}}{2 L_{j}}+\frac{Q_{j}^{2}}{2 C_{j}},
$$

the damping is provided by adding an imaginary term to the effective Hamiltonian,

$$
H_{\mathrm{damp}, j}=-i \Gamma b_{j}^{*} b_{j},
$$

and the interaction with the external field is provided by

$$
V_{\mathrm{ext}, j}=-\mathcal{E}_{j, \mathrm{ext}}^{(+)} Q_{j}^{(-)}-\Phi_{j, \mathrm{ext}}^{(+)} \frac{\phi_{j}^{(-)}}{L_{j}}+\text { c.c.. }
$$

The physical significance of the interaction term becomes clearer in the dipole approximation. When we neglect the spatial extent of the meta-atoms, the interaction potential with the external fields becomes

$$
\begin{aligned}
V_{\mathrm{ext}, j}= & -\mathbf{E}_{j, \mathrm{ext}}^{(+)}\left(\mathbf{R}_{j}, t\right) \cdot \mathbf{d}_{j}^{(-)}(t) \\
& -\mathbf{B}_{j, \mathrm{ext}}^{(+)}\left(\mathbf{R}_{j}, t\right) \cdot \mathbf{m}_{j}^{(-)}(t)+\text { c.c. },
\end{aligned}
$$

where $\mathbf{d}_{j}^{( \pm)}(t) \equiv h_{j} Q_{j}^{( \pm)}(t)$ is the electric dipole of the metaatom and

$$
\mathbf{m}_{j}^{( \pm)} \equiv \frac{A_{j}}{L_{j}} \phi_{j}^{( \pm)}(t),
$$

is an effective magnetic dipole of the meta-atom. To understand why $\mathbf{m}_{j}^{( \pm)}$can be interpreted in this way, consider the conjugate momentum expressed in terms of the self-inductance [Eq. (99)] in the limits of the RWA (namely, $\Omega / \omega_{j} \approx 1$ ),

$$
\phi_{j}^{( \pm)}=L_{j}\left(1 \pm \frac{\Gamma_{\mathrm{M}, j}}{\omega_{j}}\right) I_{j}^{( \pm)}+\Phi_{j, \mathrm{ext}}^{( \pm)} .
$$

Because $\Gamma_{\mathrm{M}, j} \ll \omega_{j}$, when the self-induced magnetic flux dominates that generated by external fields, the conjugate momentum is related to the current by

$$
\phi_{j}^{( \pm)} \approx L_{j} I_{j}^{( \pm)}
$$

and $\mathbf{m}_{j}^{\prime( \pm)} \approx \mathbf{m}_{j}^{( \pm)}$is approximately the magnetic dipole created by the current oscillation in meta-atom $j$. The effective interaction Hamiltonian [see Eq. (126)] accounts for the energy of the meta-atom electric dipole interacting with externally generated electric fields and the meta-atom's magnetic dipole interacting with externally generated magnetic fields.

The energy lost due to radiative damping is carried off by the scattered fields. The external fields contributing to the interaction $V_{\text {ext, } j}$ include fields scattered from other metaatoms in the system. In the following section, we will explore how these scattered fields drive and influence the dynamics of the meta-atoms.

\section{B. Collective interactions in the rotating wave approximation}

In this section, we examine in detail how the fields emitted externally to meta-atom $j$ drive the excitation in that meta-atom. In particular, we will see how the fields emitted or scattered from the ensemble of meta-atoms mediate interactions between them. The EM field generated externally to each meta-atom has two components: the incident field, and the fields scattered from all other meta-atoms in the system. The incident field impinges on the metamaterial driving all of its constituent meta-atoms. Each excited meta-atom, in turn, radiates an EM field which can drive other meta-atoms while undergoing multiple scattering between different resonators. In order to calculate the response of the metamaterial array to incident EM fields, we need to consider these multiple scattering processes, which produce a coupling between metaatom current oscillations. For near-resonant fields, recurrent scattering events in which the field scatters off the same meta-atom multiple times dramatically affect the coupling between closely spaced resonators.

Here, we will derive a coupled set of equations for the meta-atoms where all the multiple scattering processes are fully incorporated in the EM field induced interactions between the meta-atoms. We will then examine how the coupling can lead to a cooperative response of the metamaterial to the incident field via excitation of collective modes of current oscillation. Such modes can have either superradiant character, where the interactions enhance the radiation emitted from metamaterial, or a subradiant character, where the radiation remains trapped as it repeatedly scatters between meta-atoms leading to a suppressed collective radiative emission rate.

In order to derive a coupled set of equations for the metaatoms where the interactions are mediated by the EM fields, we consider the meta-atom mode variables $b_{j}$ and investigate their dynamics within the RWA. As stated in Sec. V A, in order for the RWA to be valid, we assume that the emission rates satisfy $\Gamma_{\mathrm{E}, j}, \Gamma_{\mathrm{M}, j}, \Gamma_{\mathrm{O}, j} \ll \Omega_{0}$ and that the driving field's bandwidth and its detuning from meta-atom resonance are small compared to the frequency of the driving field, i.e., $\delta \Omega,\left|\omega_{j}-\Omega_{0}\right| \ll \Omega_{0}$ for all meta-atoms $j$. In these limits, the external field interactions act as a slow perturbation on the fast oscillations caused by the meta-atoms' self-generated fields.

A meta-atom $j$ experiences driving from the external electric and magnetic fields. These fields induce EMFs and fluxes, which by Eq. (121), impact the dynamics of the current oscillation. The driving originates from both the incident field and from the fields scattered from all other meta-atoms $j^{\prime} \neq j$ in the system. As such, we decompose the EMF and flux into those generated directly by the incident driving, $\tilde{\mathcal{E}}_{j, \text { in }}$ and $\tilde{\Phi}_{j, \text { in }}$, and those induced by fields arriving from meta-atom $j^{\prime}, \widetilde{\mathcal{E}}_{j, j^{\prime}}$ 
and $\tilde{\Phi}_{j, j^{\prime}}$. Explicitly,

$$
\begin{aligned}
\tilde{\mathcal{E}}_{j, \mathrm{ext}} & =\tilde{\mathcal{E}}_{j, \mathrm{in}}+\sum_{j^{\prime} \neq j} \tilde{\mathcal{E}}_{j, j^{\prime}}, \\
\tilde{\Phi}_{j, \mathrm{ext}} & =\tilde{\Phi}_{j, \mathrm{in}}+\sum_{j^{\prime} \neq j} \tilde{\Phi}_{j, j^{\prime}} .
\end{aligned}
$$

The incident field directly drives each meta-atom, inducing a forcing term

$$
f_{j, \text { in }} \equiv \frac{1}{\sqrt{2}}\left(i \tilde{\mathcal{E}}_{j, \text { in }}-\omega_{j} \tilde{\Phi}_{j, \text { in }}\right),
$$

while the scattered fields produce a coupling between the resonators. Below, we will show that in the RWA, the scattered fields emanating from meta-atom $j^{\prime}$ are proportional to the amplitude $b_{j^{\prime}}$ of the oscillation in meta-atom $j^{\prime}$, and therefore that $\tilde{\mathcal{E}}_{j, j^{\prime}}$ and $\tilde{\Phi}_{j, j^{\prime}}$ are proportional to $b_{j^{\prime}}$. We will find that, by virtue of the scattered fields, the dynamics of the individual meta-atoms are coupled. The ensemble will exhibit collective modes of oscillation, each with its own frequency and radiative decay rate.

Because the incident field has a narrow bandwidth around a frequency $\Omega_{0}$, we find it convenient to define slowly varying quantities to describe the dynamics of the system. For any vector field $\mathbf{F}(\mathbf{r}, t)=\mathbf{F}^{(+)}(\mathbf{r}, t)+\mathbf{F}^{(-)}(\mathbf{r}, t)$ with positive and negative frequency components $\mathbf{F}^{(+)}$and $\mathbf{F}^{(-)}$, respectively, unless otherwise specified, we define the slowly varying envelope $\tilde{\mathbf{F}}(\mathbf{r}, t)$ of the field such that the positive frequency component

$$
\mathbf{F}^{(+)}(\mathbf{r}, t) \equiv e^{-i \Omega_{0} t} \tilde{\mathbf{F}}(\mathbf{r}, t),
$$

or equivalently in frequency space,

$$
\mathbf{F}^{(+)}(\mathbf{r}, \Omega)=\tilde{\mathbf{F}}(\mathbf{r}, \delta),
$$

where again $\delta \equiv \Omega-\Omega_{0}$ [see Eq. (115)]. For the charge and conjugate momentum on meta-atom $j$, we define the scaled slowly varying quantities $\tilde{Q}_{j}$ and $\tilde{\phi}_{j}$ such that

$$
\begin{aligned}
& \frac{Q_{j}^{(+)}(t)}{\sqrt{\omega_{j} C_{j}}} \equiv e^{-i \Omega_{0} t} \tilde{Q}_{j}(t), \\
& \frac{\phi_{j}^{(+)}(t)}{\sqrt{\omega_{j} L_{j}}} \equiv e^{-i \Omega_{0} t} \tilde{\phi}_{j}(t) .
\end{aligned}
$$

In the RWA, $\tilde{Q}_{j}$ and $\tilde{\phi}_{j}$ are trivially related to the normal variables by

$$
\begin{aligned}
& \tilde{Q}_{j}(t)=\frac{b_{j}(t)}{\sqrt{2}}, \\
& \tilde{\phi}_{j}(t)=-i \frac{b_{j}(t)}{\sqrt{2}} .
\end{aligned}
$$

Outside the RWA, $b_{j}$ contains fast oscillating components whose origins we discuss in Appendix C. In this section, however, we will assume that Eq. (137) holds. We also define the scaled current such that

$$
\sqrt{\frac{L_{j}}{\omega_{j}}} I_{j}^{(+)}(t) \equiv e^{-i \Omega_{t}} \tilde{I}_{j}(t) .
$$

The relative scale factor of the current was chosen so that, for a frequency $\delta \ll \Omega_{0}$, the Fourier components of $\tilde{\phi}_{j}$ and $\tilde{I}_{j}$ are related by

$$
\tilde{\phi}_{j}(\delta)=D_{j}\left(\Omega_{0}+\delta\right) \tilde{I}_{j}(\delta)+\tilde{\Phi}_{j, \mathrm{ext}}(\delta) .
$$

The quantity $D_{j}$ [Eq. (105)] serves as the dimensionless complex self-inductance. Because we have assumed $\Gamma_{\mathrm{M}, j} \ll$ $\Omega_{0}$ in the RWA, the quantity $D_{j} \approx 1$.

Next, we will determine the contribution of the fields scattered from each meta-atom $j^{\prime}$ to the normalized EMF, $\tilde{\mathcal{E}}_{j, j^{\prime}}$, and flux, $\tilde{\Phi}_{j, j^{\prime}}$, of meta-atom $j$. We express the scattered fields from the meta-atom $j^{\prime}$ in terms of the normalized variables $\tilde{Q}_{j^{\prime}}$ and $\tilde{I}_{j^{\prime}}$. We assume the bandwidth of the incident field is sufficiently small that the time scale over which the fields vary, $1 / \delta \Omega$, is much longer than the time it takes for light to propagate across the metamaterial sample. We then obtain the slowly varying scattered fields by substituting $\Omega_{0}$ for $\Omega$ in the radiation kernels, $\mathbf{G}$ and $\mathbf{G}_{\times}$[see Eqs. (63a) and (63b)] and exploit Eq. (134) to obtain

$$
\begin{aligned}
\tilde{\mathbf{E}}_{\mathrm{S}, j^{\prime}}= & \frac{3}{2} \sqrt{\frac{k_{0}^{3}}{6 \pi \epsilon_{0}}}\left(\frac{\Omega_{0}}{\omega_{j^{\prime}}}\right)^{3 / 2}\left[\sqrt{\Gamma_{\mathrm{E}, j^{\prime}}} \tilde{Q}_{j^{\prime}}\right. \\
& \times \int d^{3} r_{j^{\prime}} \mathbf{G}\left(\mathbf{r}-\mathbf{r}_{j^{\prime}}, \Omega_{0}\right) \cdot \frac{\mathbf{p}_{j^{\prime}}\left(\mathbf{r}_{j^{\prime}}\right)}{h_{j^{\prime}}}+\sqrt{\Gamma_{\mathrm{M}, j^{\prime}}} \tilde{I}_{j^{\prime}} \\
& \left.\times \int d^{3} r_{j^{\prime}} \mathbf{G}_{\times}\left(\mathbf{r}-\mathbf{r}_{j^{\prime}}, \Omega_{0}\right) \cdot \frac{\mathbf{w}_{j^{\prime}}\left(\mathbf{r}_{j^{\prime}}\right)}{A_{j^{\prime}}}\right]
\end{aligned}
$$

and

$$
\begin{aligned}
\tilde{\mathbf{H}}_{\mathrm{S}, j^{\prime}}= & \frac{3}{2} \sqrt{\frac{k_{0}^{3}}{6 \pi \mu_{0}}}\left(\frac{\Omega_{0}}{\omega_{j^{\prime}}}\right)^{3 / 2}\left[\sqrt{\Gamma_{\mathrm{M}, j^{\prime}}}{\tilde{j^{\prime}}}_{j^{\prime}}\right. \\
& \times \int d^{3} r_{j^{\prime}} \mathbf{G}^{(+)}\left(\mathbf{r}-\mathbf{r}_{j^{\prime}}, \Omega_{0}\right) \cdot \frac{\mathbf{w}_{j^{\prime}}\left(\mathbf{r}_{j^{\prime}}\right)}{A_{j^{\prime}}}-\sqrt{\Gamma_{\mathrm{E}, j^{\prime}}} \tilde{Q}_{j^{\prime}} \\
& \left.\times \int d^{3} r_{j^{\prime}} \mathbf{G}_{\times}^{(+)}\left(\mathbf{r}-\mathbf{r}_{j^{\prime}}, \Omega_{0}\right) \cdot \frac{\mathbf{p}_{j^{\prime}}\left(\mathbf{r}_{j^{\prime}}\right)}{h_{j^{\prime}}}\right] .
\end{aligned}
$$

The amplitude of the electric and magnetic fields emitted by the electric dipole of meta-atom $j^{\prime}$, driven by $\tilde{Q}_{j^{\prime}}$, scale with $\sqrt{\Gamma_{\mathrm{E}, j^{\prime}}}$. Similarly, the fields emitted by the magnetic dipole of meta-atom $j^{\prime}$, driven by $\tilde{I}_{j^{\prime}}$, scale with $\sqrt{\Gamma_{\mathrm{M}, j^{\prime}}}$.

These scattered fields provide a portion of the slowly varying EMF

$$
\tilde{\mathcal{E}}_{j, j^{\prime}}=\frac{1}{\sqrt{\omega_{j} L_{j}}} \int d^{3} r_{j} \mathbf{p}_{j}\left(\mathbf{r}_{j}\right) \cdot \tilde{\mathbf{E}}_{\mathrm{S}, j^{\prime}}\left(\mathbf{r}_{j}\right)
$$

and flux

$$
\tilde{\Phi}_{j, j^{\prime}}=\frac{\mu_{0}}{\sqrt{\omega_{j} L_{j}}} \int d^{3} r_{j} \mathbf{w}_{j}\left(\mathbf{r}_{j}\right) \cdot \tilde{\mathbf{H}}_{\mathrm{S}, j^{\prime}}\left(\mathbf{r}_{j}\right)
$$

at meta-atom $j$. Substitution of Eqs. (140) and (141) into the expressions for EMF and flux gives

$$
\begin{aligned}
\tilde{\mathcal{E}}_{j, j^{\prime}}= & \left(\frac{\Omega_{0}}{\sqrt{\omega_{j} \omega_{j^{\prime}}}}\right)^{3}\left[\sqrt{\Gamma_{\mathrm{E}, j} \Gamma_{\mathrm{E}, j^{\prime}}}\left[\mathcal{G}_{\mathrm{E}}\left(\Omega_{0}\right)\right]_{j, j^{\prime}} \tilde{Q}_{j^{\prime}}\right. \\
& \left.+\sqrt{\Gamma_{\mathrm{E}, j} \Gamma_{\mathrm{M}, j^{\prime}}}\left[\mathcal{G}_{\times}\left(\Omega_{0}\right)\right]_{j, j^{\prime}} \tilde{I}_{j^{\prime}}\right] \\
\tilde{\Phi}_{j, j^{\prime}}= & \frac{1}{\omega_{j}}\left(\frac{\Omega_{0}}{\sqrt{\omega_{j} \omega_{j^{\prime}}}}\right)^{3}\left[\sqrt{\Gamma_{\mathrm{M}, j} \Gamma_{\mathrm{M}, j^{\prime}}}\left[\mathcal{G}_{\mathrm{M}}\left(\Omega_{0}\right)\right]_{j, j^{\prime}} \tilde{I}_{j^{\prime}}\right. \\
& \left.\left.-\sqrt{\Gamma_{\mathrm{M}, j} \Gamma_{\mathrm{E}, j^{\prime}}} \mathcal{G}_{\times}^{T}\left(\Omega_{0}\right)\right]_{j, j^{\prime}} \tilde{Q}_{j^{\prime}}\right],
\end{aligned}
$$


where the matrices $\mathcal{G}_{\mathrm{E}}, \mathcal{G}_{\mathrm{M}}$, and $\mathcal{G}_{\times}$determine how the meta-atoms' geometries and relative orientations influence the respective contributions of the scattered electric fields to the EMFs, the scattered magnetic fields to the fluxes, and the scattered electric (magnetic) fields the fluxes (EMFs). These matrices have zero diagonal elements and off-diagonal elements given by

$$
\begin{aligned}
& {\left[\mathcal{G}_{\mathrm{E}}(\Omega)\right]_{j, j^{\prime}}} \\
& =\frac{3}{2} \int d^{3} r_{j} \int d^{3} r_{j^{\prime}} \frac{\mathbf{p}_{j}\left(\mathbf{r}_{j}\right)}{h_{j}} \cdot \mathbf{G}\left(\mathbf{r}_{j}-\mathbf{r}_{j^{\prime}}, \Omega\right) \cdot \frac{\mathbf{p}_{j^{\prime}}\left(\mathbf{r}_{j^{\prime}}\right)}{h_{j^{\prime}}}, \\
& {\left[\mathcal{G}_{\mathrm{M}}(\Omega)\right]_{j, j^{\prime}}} \\
& \quad=\frac{3}{2} \int d^{3} r_{j} \int d^{3} r_{j^{\prime}} \frac{\mathbf{w}_{j}\left(\mathbf{r}_{j}\right)}{A_{j}} \cdot \mathbf{G}\left(\mathbf{r}_{j}-\mathbf{r}_{j^{\prime}}, \Omega\right) \cdot \frac{\mathbf{w}_{j^{\prime}}\left(\mathbf{r}_{j^{\prime}}\right)}{A_{j^{\prime}}}, \\
& {\left[\mathcal{G}_{\times}(\Omega)\right]_{j, j^{\prime}}} \\
& \quad=\frac{3}{2} \int d^{3} r_{j} \int d^{3} r_{j^{\prime}} \frac{\mathbf{p}_{j}\left(\mathbf{r}_{j}\right)}{h_{j}} \cdot \mathbf{G}_{\times}\left(\mathbf{r}_{j}-\mathbf{r}_{j^{\prime}}, \Omega\right) \cdot \frac{\mathbf{w}_{j^{\prime}}\left(\mathbf{r}_{j^{\prime}}\right)}{A_{j^{\prime}}} .
\end{aligned}
$$

When the separation between two meta-atoms is much greater than the spatial extent of the individual elements, these geometrical factors depend exclusively on the relative positions and orientations of the meta-atoms' electric and magnetic dipoles. Explicitly, in that limit,

$$
\begin{aligned}
{\left[\mathcal{G}_{\mathrm{E}}(\Omega)\right]_{j, j^{\prime}} } & =\frac{3}{2} \hat{\mathbf{d}}_{j} \cdot \mathbf{G}\left(\mathbf{R}_{j}-\mathbf{R}_{j^{\prime}}, \Omega\right) \cdot \hat{\mathbf{d}}_{j^{\prime}}, \\
{\left[\mathcal{G}_{\mathrm{M}}(\Omega)\right]_{j, j^{\prime}} } & =\frac{3}{2} \hat{\mathbf{m}}_{j} \cdot \mathbf{G}\left(\mathbf{R}_{j}-\mathbf{R}_{j^{\prime}}, \Omega\right) \cdot \hat{\mathbf{m}}_{j^{\prime}}, \\
{\left[\mathcal{G}_{\times}(\Omega)\right]_{j, j^{\prime}} } & =\frac{3}{2} \hat{\mathbf{d}}_{j} \cdot \mathbf{G}_{\times}\left(\mathbf{R}_{j}-\mathbf{R}_{j^{\prime}}, \Omega\right) \cdot \hat{\mathbf{m}}_{j^{\prime}} \cdot
\end{aligned}
$$

The contribution of the electric field scattered by meta-atom $j^{\prime}$ to the EMF, $\tilde{\mathcal{E}}_{j, j^{\prime}}$, scales with the geometric mean of the electric dipole emission rates of the two meta-atoms, $\sqrt{\Gamma_{\mathrm{E}, j} \Gamma_{\mathrm{E}, j^{\prime}}}$. Similarly, the magnetic field of element $j^{\prime}$ contributes to the flux $\tilde{\Phi}_{j, j^{\prime}}$ with a strength proportional to $\sqrt{\Gamma_{\mathrm{M}, j} \Gamma_{\mathrm{M}, j^{\prime}}}$. When the meta-atoms are sufficiently far away from one and other, the electric field emitted by the magnetic dipoles and the magnetic field emitted by the electric dipoles provide a significant contribution to $\tilde{\mathcal{E}}_{j, j^{\prime}}$ and $\tilde{\Phi}_{j, j^{\prime}}$ that scale with $\sqrt{\Gamma_{\mathrm{E}, j} \Gamma_{\mathrm{M}, j^{\prime}}}$ and $\sqrt{\Gamma_{\mathrm{M}, j} \Gamma_{\mathrm{E}, j^{\prime}}}$, respectively.

We have set out to obtain coupled equations of motion for the meta-atom normal variables $b_{j}$ mediated by the EM field. We have obtained contributions to the EMF and flux that are driven by charges $\tilde{Q}_{j}$ and currents $\tilde{I}_{j}$. However, only $\tilde{Q}_{j}$ and conjugate momenta $\tilde{\phi}_{j}$ are trivially related to these normal variables [see Eq. (137)]. The current, on the other hand obeys the more complex relationship

$$
\tilde{I}_{j^{\prime}}=-i \frac{b_{j^{\prime}}}{\sqrt{2}}-\sum_{j^{\prime \prime} \neq j^{\prime}} \tilde{\Phi}_{j^{\prime}, j^{\prime \prime}}-\tilde{\Phi}_{j^{\prime}, \text { in }} .
$$

One can thus use Eqs. (137) and (148) to express $\tilde{\mathcal{E}}_{j, j^{\prime}}$ and $\tilde{\Phi}_{j, j^{\prime}}$ in terms of the normal variables $b_{j^{\prime}}$. We note, however, from Eq. (145), that $\tilde{\Phi}_{j^{\prime}, j^{\prime \prime}}$ contains contributions that scale as $\sqrt{\Gamma_{\mathrm{M}, j^{\prime}} \Gamma_{\mathrm{M}, j^{\prime \prime}}} / \omega_{j}$ and $\sqrt{\Gamma_{\mathrm{M}, j^{\prime}} \Gamma_{\mathrm{E}, j^{\prime \prime}}} / \omega_{j}$, which under the conditions of the RWA, are much less than 1. Furthermore, $\tilde{I}_{j}$ contains a contribution from the incident field flux $\tilde{\Phi}_{j, \text { in }}$. The contribution of the incident flux to $\tilde{I}_{j}$ can also be ignored to lowest order since it is about $\Gamma_{j} / \omega_{j}$ times the direct contribution of the incident flux to the direct driving $f_{j \text {,in }}$. So, to determine $\tilde{\mathcal{E}}_{j, j^{\prime}}$ and $\tilde{\Phi}_{j, j^{\prime}}$ to lowest order in $\Gamma_{j} / \omega_{j}$, we therefore exploit the approximate relationship $\tilde{I}_{j^{\prime}} \approx \tilde{\phi}_{j^{\prime}} \approx-i b_{j^{\prime}} / \sqrt{2}$.

Having computed the contributions of the scattered fields to the EMF and flux of an individual meta-atom, we find that these scattered fields produce a coupling between meta-atoms in the oscillator equations of motion. Substituting the EMF and flux into Eq. (121), we find the evolution of the column vector $b$ of normal variables is governed by

$$
\dot{\mathrm{b}}=\mathcal{C} \mathrm{b}+\mathrm{f}_{\mathrm{in}},
$$

where we have introduced the following notation for $\mathrm{b}$ and for the driving $f_{\text {in }}$ caused by the incident field:

$$
\mathrm{b} \equiv\left(\begin{array}{c}
b_{1} \\
b_{2} \\
\vdots \\
b_{N}
\end{array}\right), \quad \mathrm{f}_{\mathrm{in}} \equiv\left(\begin{array}{c}
f_{1, \text { in }} \\
f_{2, \text { in }} \\
\vdots \\
f_{N, \text { in }}
\end{array}\right) .
$$

The coupling matrix $\mathcal{C}$ is given to lowest order in $\Gamma_{\mathrm{E}, j} / \omega_{j^{\prime}}$ and $\Gamma_{\mathrm{M}, j} / \omega_{j^{\prime}}$ by

$$
\begin{aligned}
\mathcal{C}= & -i \Delta-\frac{1}{2} \Upsilon+\frac{1}{2}\left(i \Upsilon_{\mathrm{E}}^{\frac{1}{2}} \mathcal{G}_{\mathrm{E}} \Upsilon_{\mathrm{E}}^{\frac{1}{2}}+i \Upsilon_{\mathrm{M}}^{\frac{1}{2}} \mathcal{G}_{\mathrm{M}} \Upsilon_{\mathrm{M}}^{\frac{1}{2}}\right. \\
& \left.+\Upsilon_{\mathrm{E}}^{\frac{1}{2}} \mathcal{G}_{\times} \Upsilon_{\mathrm{M}}^{\frac{1}{2}}+\Upsilon_{\mathrm{M}}^{\frac{1}{2}} \mathcal{G}_{\times}^{T} \Upsilon_{\mathrm{E}}^{\frac{1}{2}}\right) .
\end{aligned}
$$

Here, the detunings of the incident field from the meta-atom resonances are contained in the diagonal matrix $\Delta$ with elements

$$
\Delta_{j, j} \equiv \omega_{j}-\Omega_{0} .
$$

Moreover, the meta-atom emission rates are incorporated in the diagonal matrices $\Upsilon_{E}, \Upsilon_{M}$, and $\Upsilon_{O}$ with elements

$$
\begin{aligned}
{\left[\Upsilon_{\mathrm{E}}\right]_{j, j} } & \equiv \Gamma_{\mathrm{E}, j}, \\
{\left[\Upsilon_{\mathrm{M}}\right]_{j, j} } & \equiv \Gamma_{\mathrm{M}, j}, \\
{\left[\Upsilon_{\mathrm{O}}\right]_{j, j} } & \equiv \Gamma_{\mathrm{O}, j},
\end{aligned}
$$

respectively, and we have defined $\Upsilon \equiv \Upsilon_{\mathrm{E}}+\Upsilon_{\mathrm{M}}+\Upsilon_{\mathrm{O}}$.

The interaction matrix $\mathcal{C}$ accounts for electric dipole-dipole interactions, magnetic dipole-dipole interactions, as well as interactions between electric and magnetic dipoles that arise from magnetic (electric) fields emitted by electric (magnetic) dipoles. The diagonal elements of $\mathcal{C}$ result from interactions with the self-generated fields and give rise to the meta-atoms' resonance frequencies and radiative emission rates.

In the RWA, the dynamic equation [see Eq. (149)] encapsulates all the multiple scattering processes between the different meta-atoms. These are described by the interaction terms in the matrix $\mathcal{C}$, mediated by the scattered EM fields. The coupled set of equations implies a system of $N$ meta-atoms possesses $N$ collective modes of excitation. These modes correspond to the eigenvectors of the matrix $\mathcal{C}$. For each collective eigenmode, we have collective radiative resonance linewidths and resonance frequencies that are represented by the eigenvalues of $\mathcal{C}$. A strong coupling between the resonators can lead to a cooperative response of the metamaterial sample to the EM fields, resulting in collective decay rates which are substantially different from those of a single, isolated meta-atom. The interactions can either enhance radiative emission, producing a 
superradiant mode, or suppress emission, yielding a subradiant decay rate. We will illustrate the effect of a cooperative response of a 2D metamaterial array in Sec. VI by considering an example of closely-spaced split ring resonators. We find that even in a relatively small sample the strong coupling leads to a dramatic resonance linewidth narrowing of five orders of magnitude and to a broad distribution of radiative decay rates.

In order to illustrate the coupling of an incoming field to collective modes, suppose the incident field is engineered so that it only excites the $i$ th collective mode, and then is suddenly turned off. The collective excitation is then distributed over the sample according to the eigenvector $\mathrm{v}_{i}$ of $\mathcal{C}$. Due to the repeatedly scattered fields that couple the meta-atoms, the excitation oscillates at its resonance frequency given by the eigenvalue $\lambda_{i}$,

$$
\Omega_{i} \equiv \Omega_{0}-\operatorname{Im}\left(\lambda_{i}\right),
$$

and the amplitude of oscillations decay at a rate

$$
\gamma_{i} \equiv-2 \operatorname{Re}\left(\lambda_{i}\right) \text {. }
$$

As radiation leaks out of the collective excitation and energy dissipates through ohmic losses. The vector of normal variables then evolves as

$$
\mathrm{b}(t) \propto \exp \left\{\left[-i\left(\Omega_{i}-\Omega_{0}\right)-\frac{\gamma_{i}}{2}\right] t\right\} \mathrm{v}_{i} .
$$

The nature of collective modes could also allow one to engineer a cooperative response of the metamaterial to the incident field, addressing linear combinations of modes by shaping the incident field's profile, or adjusting its frequency. Engineering of the collective response may then be used, for example, to excite isolated subwavelength hot spots in a metamaterial. ${ }^{39}$

\section{Concluding remarks}

In this section, we saw how the interaction of individual meta-atoms with the EM field governs the collective dynamics of an ensemble of meta-atoms that make up a metamaterial. Each meta-atom experiences the influence of its current oscillation's self-generated field, the field incident on the metamaterial, and the fields scattered from all other metaatoms in the system. We explored the influence of the selfgenerated fields in Sec. V A. In the RWA, the self-generated field dominates meta-atom dynamics. Each meta-atom can be seen as an effective RLC circuit which experiences damping due to electric and magnetic dipole radiation carrying energy away from the meta-atom. On the other hand, fields generated externally to the meta-atom, i.e., the incident field and the fields radiated from all other meta-atoms in the metamaterial, drive the current oscillations in each meta-atom. In Sec. V B, we saw how the fields scattered by each meta-atom mediate interactions between them. Fields emitted by one meta-atom drive the current oscillations in all the others, producing the dynamic inter-meta-atom coupling in Eq. (149). While Appendix C, develops a formalism to account for arbitrarily strong interactions, in this section, we have gained a significant physical insight in the RWA in which we assume the metaatoms' interact much more strongly with their self-generated fields than with the fields generated externally.
In the following section, we will apply this formalism to examine collective modes in an example metamaterial: an array of symmetric split ring resonators. This system will illustrate the vital role cooperative interactions can play in the dynamics of a metamaterial composed of closely spaced plasmonic resonators. A metamaterial of $N$ resonators will have $N$ collective modes of current oscillation, each with its own resonance frequency and radiative emission rate. Both of these quantities strongly influence how a given mode can be excited. The cooperative interactions lead to a broad distribution of collective decay rates indicating strongly superradiant or subradiant modes.

\section{AN ENSEMBLE OF SYMMETRIC SPLIT RING RESONATORS}

In this section, we apply the formalism developed in this article to a metamaterial composed of split ring resonators (SRRs). As the name suggests, these resonators are composed of loops with segments that have been removed. Owing to the curvature of the elements, current oscillations within SRRs can exhibit both an electric and a magnetic response. Variations of these resonators have been used to produce metamaterials that exhibit, e.g., negative indices of refraction. ${ }^{1,2}$ Here, we consider a particular realization of the SRR in which a single ring is cut into two disconnected concentric circular arcs of equal length. We then study the SRR metamolecule by assuming that the halves each form a meta-atom that supports a single mode of current oscillation. The two halves could either oscillate in phase, producing a net electric dipole, or out of phase, producing a net magnetic dipole.

In addition to active studies of metamaterial arrays of SRRs, there has also been an increasing interest in fabricating metamaterials consisting of split ring resonators in which the symmetry between the two disconnected halves has been broken, e.g., by making one of them longer. Sheets of asymmetric split ring resonators (ASRs) have been shown to exhibit transmission resonances ${ }^{52}$ corresponding to excitations in which all magnetic dipoles in the sheet oscillated in phase. The quality factor of this resonance, however, was shown to depend strongly on the number of ASRs in the system. ${ }^{32}$ Furthermore, artificially adjusted disorder in the positions of the unit-cell resonators was observed to destroy the resonance. ${ }^{34}$ If interactions mediated by the EM fields were not important, and the ASRs behaved independently, system size or positional disorder of the system would have little effect on the metamaterial response to the EM fields. These experimental observations provide ample evidence for the vital role collective interactions play in this particular metamaterial.

Here, we employ the formalism describing collective interactions to an ensemble of SRRs in the RWA. We describe a single SRR in Sec. VI A, while we examine the properties of collective modes of SRRs in a lattice in Sec. VIB.

\section{A. The symmetric SRR}

We begin by describing the interaction of a single SRR unit-cell resonator with incident EM fields. This particular realization of an SRR metamolecule consists of two metaatoms formed by two concentric circular arcs labeled by 


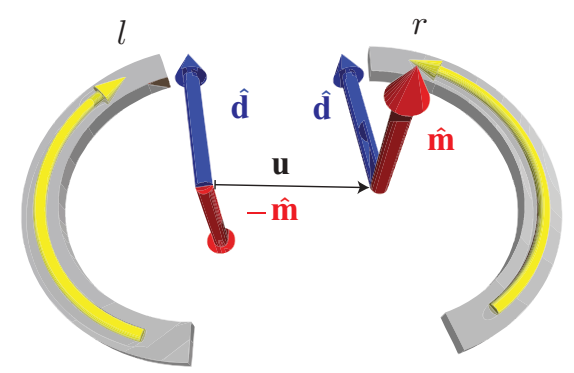

FIG. 1. (Color online) A schematic illustration of a split ring resonator. An excitation in the left meta-atom $(l)$ produces an oscillating electric dipole (indicated by the blue arrow) in the direction $\hat{\mathbf{d}}$ and a magnetic dipole (indicated by the red arrow) in the direction $-\hat{\mathbf{m}}$, while an excitation in the right meta-atom $(r)$ produces an electric dipole in the direction $\hat{\mathbf{d}}$ and a magnetic dipole in the direction $\hat{\mathbf{m}}$. The meta-atoms, in the point source approximation, are separated by a vector $\mathbf{u}$. When the meta-atoms are excited in phase, the electric dipoles reinforce each other and the magnetic dipoles cancel out.

$j \in\{l, r\}$ (for "left" and "right"), as shown in Fig. 1. This metamolecule possesses reflection symmetry about a central plane.

To illustrate this qualitative physical behavior of an SRR, we approximate the meta-atoms as two point sources separated by $\mathbf{u} \equiv \mathbf{R}_{r}-\mathbf{R}_{l}$ (see Fig. 1). The current oscillations in metaatoms produce electric dipoles with orientation $\hat{\mathbf{d}}_{r}=\hat{\mathbf{d}}_{l}=\hat{\mathbf{d}}$ associated with charge oscillating between the ends of the arcs. Owing to the curvature of the meta-atoms, these currents also produce magnetic dipoles with opposite orientations $\hat{\mathbf{m}}_{r}=-\hat{\mathbf{m}}_{l}=\hat{\mathbf{m}}$. The generated electric dipoles lie in the plane of the SRR and are perpendicular to the displacement between the meta-atoms $(\hat{\mathbf{d}} \perp \hat{\mathbf{u}})$. The generated magnetic dipoles, on the other hand, point out of the plane in which the SRR resides $(\hat{\mathbf{m}} \perp \hat{\mathbf{u}}, \hat{\mathbf{d}})$. Each meta-atom in isolation supports a single mode of oscillation with resonance frequency $\omega_{0}$. Here, we consider a resonant driving with the frequency of the incident field satisfying $\Omega_{0}=\omega_{0}$. For simplicity, we also assume each element possesses identical radiative and thermal decay rates $\Gamma_{\mathrm{E} / \mathrm{M} / \mathrm{O}, l}=\Gamma_{\mathrm{E} / \mathrm{M} / \mathrm{O}, r}=\Gamma_{\mathrm{E} / \mathrm{M} / \mathrm{O}}$.

In the RWA, the normal variables $b_{r}$ and $b_{l}$ [see Eq. (109) with $j \in\{r, l\}]$ describe the states of the right and left halves, respectively, of a single SRR metamolecule in isolation. We may now apply the previously developed theory for the EM field mediated interactions between meta-atoms to a single SRR unit-cell resonator consisting of these two meta-atoms. According to Eq. (149), the normal variables $b_{r}$ and $b_{l}$ are coupled by the EM fields as

$$
\left(\begin{array}{c}
\dot{b}_{r} \\
\dot{b}_{l}
\end{array}\right)=\mathcal{C}_{\text {SRR }}\left(\begin{array}{c}
b_{r} \\
b_{l}
\end{array}\right)+\left(\begin{array}{c}
f_{r, \text { in }} \\
f_{l, \text { in }}
\end{array}\right) .
$$

Here, $\mathcal{C}_{\text {SRR }}$ denotes the specific coupling matrix in this case between the two meta-atoms, as described in detail below. The incident field impinging on the SRR produces the driving terms $f_{j, \text { in }}$ for each meta-atom $j=l, r$ [see Eq. (132)]. Considering the meta-atoms as point emitters, the incident field excites their electric and magnetic dipoles resulting in the simplified driving terms:

$$
\begin{aligned}
f_{r, \text { in }} & =i h \frac{\hat{\mathbf{d}} \cdot \tilde{\mathbf{E}}\left(\mathbf{R}_{r}, t\right)}{\sqrt{2 \omega_{0} L}}-\omega_{0} A \frac{\hat{\mathbf{m}} \cdot \tilde{\mathbf{B}}\left(\mathbf{R}_{r}, t\right)}{\sqrt{2 \omega_{0} L}}, \\
f_{l, \text { in }} & =i h \frac{\hat{\mathbf{d}} \cdot \tilde{\mathbf{E}}\left(\mathbf{R}_{l}, t\right)}{\sqrt{2 \omega_{0} L}}+\omega_{0} A \frac{\hat{\mathbf{m}} \cdot \tilde{\mathbf{B}}\left(\mathbf{R}_{l}, t\right)}{\sqrt{2 \omega_{0} L}} .
\end{aligned}
$$

The quantity $h$ is an effective length along which charge flows to form the meta-atoms' electric dipoles and is related to $\Gamma_{\mathrm{E}}$ through Eq. (103). Similarly, $A$ is an effective area that indicates the strength of the magnetic dipole interaction and is related to the magnetic dipole emission rate $\Gamma_{M}$ through Eq. (104) and $L$ is the self-inductance of each meta-atom. Once excited, each half of the SRR scatters both electric and magnetic fields. These fields then impact the other meta-atom, driving its electric and magnetic dipoles. Repeated absorption and reemission of scattered fields produces a dynamic interaction between the two halves of the SRR. From Eq. (151), the coupling matrix governing the interaction is given by

$$
\mathcal{C}_{\mathrm{SRR}}=\left(\begin{array}{cc}
-\Gamma / 2 & i \mathrm{~d} \Gamma G-\bar{\Gamma} S \\
i \mathrm{~d} \Gamma G-\bar{\Gamma} S & -\Gamma / 2
\end{array}\right),
$$

where a single meta-atom has a total decay rate

$$
\Gamma \equiv \Gamma_{\mathrm{E}}+\Gamma_{\mathrm{M}}+\Gamma_{\mathrm{O}}
$$

appearing in the diagonal elements of $\mathcal{C}_{\mathrm{SRR}}$, and we have defined

$$
\begin{gathered}
\bar{\Gamma} \equiv \sqrt{\Gamma_{\mathrm{E}} \Gamma_{\mathrm{M}}}, \\
\mathrm{d} \Gamma \equiv \Gamma_{\mathrm{E}}-\Gamma_{\mathrm{M}} .
\end{gathered}
$$

Coupling between the two halves of the SRR, represented by the off-diagonal elements of $\mathcal{C}_{\mathrm{SRR}}$, arises from interactions between the meta-atoms' electric dipoles, the meta-atoms' magnetic dipoles as well as a cross interaction between the electric dipole of one meta-atom and the magnetic dipole of the other. The strength of the electric and magnetic dipole-dipole interactions is proportional to the radiative decay rates $\Gamma_{\mathrm{E}}$ and $\Gamma_{\mathrm{M}}$, respectively. These dipole-dipole interactions also depend on the spacing between the meta-atoms and the relative orientations of the dipoles. This geometrical dependence shows up in the factor

$$
G \equiv \frac{3}{4} \hat{\mathbf{d}} \cdot \mathbf{G}\left(\mathbf{u}, \Omega_{0}\right) \cdot \hat{\mathbf{d}}=\frac{3}{4} \hat{\mathbf{m}} \cdot \mathbf{G}\left(\mathbf{u}, \Omega_{0}\right) \cdot \hat{\mathbf{m}} .
$$

Notice that because identical meta-atom excitations (i.e., when $b_{l}=b_{r}$ ) produce parallel electric dipoles, but antiparallel magnetic dipoles, the electric and magnetic dipole interactions work against each other; the strength of interaction arising from the geometrical factor $G$ is proportional to $\Gamma_{\mathrm{E}}-\Gamma_{\mathrm{M}}$. An additional interaction arises from the electric dipoles interacting with fields scattered from the magnetic dipoles and vice versa. The geometric mean of the radiative decay rates, $\bar{\Gamma}$ [see Eq. (162)], governs the strength of this interaction. Relative orientations of the electric dipole of the left (right) meta-atom and the magnetic dipole of the right (left) meta-atom appear in the geometrical factor

$$
S \equiv \frac{3}{4} \hat{\mathbf{d}} \cdot \mathbf{G}_{\times}\left(\mathbf{u}, \Omega_{0}\right) \cdot \hat{\mathbf{m}}_{r},
$$

To analyze the collective modes of the SRR, we consider the dynamics of symmetric $c_{+}$and antisymmetric $c_{-}$modes 
of oscillation defined by

$$
c_{ \pm} \equiv \frac{1}{\sqrt{2}}\left(b_{r} \pm b_{l}\right) .
$$

These symmetric and antisymmetric variables represent the eigenmodes of the SRR. From the dynamic equation [see Eq. (149)] and the SRR coupling matrix [see Eq. (160)], one finds

$$
\frac{d}{d t} c_{ \pm}=\left(-\frac{\gamma_{ \pm}}{2} \mp i \Delta_{\mathrm{SRR}}\right) c_{ \pm}+F_{ \pm},
$$

where an incident field produces the driving terms

$$
F_{ \pm}=\frac{1}{\sqrt{2}}\left(f_{r, \text { in }} \pm f_{l, \text { in }}\right) .
$$

The interaction between the elements produces the decay rates $\gamma_{ \pm}$and shifts the resonance frequencies of the symmetric and antisymmetric modes by equal and opposite amounts, $\Delta_{\mathrm{SRR}}$ :

$$
\begin{aligned}
\gamma_{ \pm}= & \Gamma_{\mathrm{E}}[1 \pm 2 \operatorname{Im}(G)] \\
& +\Gamma_{\mathrm{M}}[1 \mp 2 \operatorname{Im}(G)]+2 \bar{\Gamma} \operatorname{Re}(S)+\Gamma_{\mathrm{O}}, \\
\Delta_{\mathrm{SRR}} & =-2 \operatorname{Re}(G)\left(\Gamma_{\mathrm{E}}-\Gamma_{\mathrm{M}}\right)-2 \bar{\Gamma} \operatorname{Im}(S) .
\end{aligned}
$$

An analogy can be drawn between these metamolecular current oscillations and atomic or molecular energy levels. ${ }^{48,49}$ The symmetric and antisymmetric modes have respective resonance frequencies $\omega_{0}+\Delta_{\mathrm{SRR}}$ and $\omega_{0}-\Delta_{\mathrm{SRR}}$. When excited, the symmetric mode decays at a rate $\gamma_{+}$, while an excitation of the antisymmetric mode decays at a rate $\gamma_{-}$.

Excitation of the symmetric mode $\left(c_{+}\right)$produces a net electric dipole since the individual meta-atom electric dipoles oscillate in phase while the meta-atom magnetic dipoles approximately cancel each other out. Similarly, excitation of the antisymmetric mode $\left(c_{-}\right)$produces a net magnetic dipole and the net effect of the electric dipole approximately cancels out. The symmetric and antisymmetric excitations will thus be referred to electric and magnetic dipole excitations, respectively. When the spacing between the arcs $u \ll \lambda$, the decay rates simplify to

$$
\begin{aligned}
& \gamma_{+} \approx 2 \Gamma_{\mathrm{E}}+\Gamma_{\mathrm{O}}, \\
& \gamma_{-} \approx 2 \Gamma_{\mathrm{M}}+\Gamma_{\mathrm{O}} .
\end{aligned}
$$

The electric mode loses energy via electric dipole radiation, while the magnetic mode emits magnetic dipole radiation. In the absence of magnetic dipole interactions, the symmetric and antisymmetric modes are analogous to superradiant and subradiant states in a pair of closely spaced two-level atoms: when the two-level atoms are excited in phase, the radiative emission rate is enhanced, and it is suppressed when the atoms are excited out of phase. Furthermore, in the SRR metamolecule, the electric and magnetic modes are driven purely by the electric and magnetic fields, respectively, with $F_{+} \propto \hat{\mathbf{d}} \cdot \tilde{\mathbf{E}}_{\text {in }}(\mathbf{R}, t)$ and $F_{-} \propto \hat{\mathbf{m}} \cdot \tilde{\mathbf{B}}_{\text {in }}(\mathbf{R}, t)$, where $\mathbf{R}$ denotes the center of mass coordinate of the SRR.

When more than one SRR is present, radiation emitted from one SRR impacts and drives oscillations in another. The resulting interactions produce collective modes of oscillation for the whole system. We examine this collective behavior in the following section.

\section{B. Collective modes in an ensemble of symmetric split rings}

Having discussed how EM field induced interactions arise between two meta-atoms in a single SRR metamolecule, we now explore how a collection of metamolecules can behave in concert when brought together to form a metamaterial. As an example, we consider a 2D $N_{x} \times N_{y}$ array of SRRs arranged in a square lattice with lattice vectors $\mathbf{a}_{1}=a \hat{\mathbf{e}}_{x}$ and $\mathbf{a}_{2}=a \hat{\mathbf{e}}_{y}$. This finite array resides in a region with free space (as opposed to, e.g., periodic) boundary conditions. A single SRR occupies each unit cell of the lattice. They are oriented such that symmetric oscillations produce electric dipoles along the direction $\hat{\mathbf{d}}=\hat{\mathbf{e}}_{y}$, and antisymmetric oscillations produce magnetic dipoles pointing out of the lattice in the direction $\hat{\mathbf{m}}=\hat{\mathbf{e}}_{z}$. In this section, we quantify the collective interactions by examining the collective eigenmodes of the system and showing how the interactions can lead to strongly modified radiative emission rates. We also illustrate from this model how a subwavelength intermolecular spacing enhances the collective behavior of the system. In particular, we find that a subwavelength lattice spacing produces a much broader distribution of subradiant and superradiant collective decay rates.

While an SRR in isolation possesses two modes with two collective resonance frequencies and two decay rates, the presence of interactions in an ensemble can produce a broad distribution of collective linewidths. The lattice of $N_{x} \times N_{y}$ SRRs possesses $2 N_{x} N_{y}$ collective modes of oscillation, where the $i$ th mode corresponds to an eigenvector $\mathrm{v}_{i}$ of the interaction matrix $\mathcal{C}$ [see Eq. (151)]. The resonance frequency of this collective mode is shifted from $\Omega_{0}$ by $\delta_{i} \equiv \Omega_{i}-\Omega_{0}$ and has a collective decay rate $\gamma_{i}$. These are given in terms of the mode's eigenvalue $\lambda_{i}$ as

$$
\begin{aligned}
& \delta_{i}=-\operatorname{Im} \lambda_{i}, \\
& \gamma_{i}=-2 \operatorname{Re} \lambda_{i},
\end{aligned}
$$

respectively. Here, we consider an ensemble of SRRs whose elements have equal single-meta-atom electric and magnetic decay rates $\Gamma_{\mathrm{E}}=\Gamma_{\mathrm{M}}$, and we take the separation between constituent meta-atoms of an SRR to be $u=0.12 \lambda$. Because the thermal losses are equal in all meta-atoms, their presence would add to the decay rates of each collective mode equally. Since here we are interested in how interactions modify collective radiative decay rates, we take the ohmic loss rate to be zero in this section.

We numerically calculate all the eigenmodes of the system that are modified by the multiple scattering processes. Figure 2 illustrates how interactions mediated by the EM field tend to broaden the distribution of collective linewidths in a $33 \times 33$ lattice of SRRs. In Fig. 2(a), where the lattice spacing is $a=0.5 \lambda$, the radiative emission rates range from the very subradiant $1.2 \times 10^{-5} \Gamma$ to the superradiant $11 \Gamma$, where $\Gamma$ is the decay rate of a single meta-atom in isolation. Figure 2(b), on the other hand, illustrates how the collective effects are diminished when the lattice spacing $a=1.4 \lambda$ exceeds a wavelength. The distribution of decay rates is considerably narrower with the decreased inter-SRR interactions associated with lattice spacings exceeding a wavelength. Although the effects of collective interactions are significantly reduced, they 

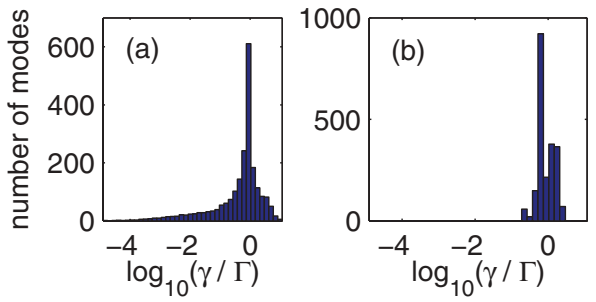

FIG. 2. (Color online) The distributions of collective radiative emission rates in a $33 \times 33$ square array of SRRs. The distribution is represented as a histogram of the $\log _{10}$ of the collective emission rates. (a) The distribution for an ensemble of SRRs with lattice spacing $a=0.5 \lambda$. (b) The distribution when the lattice spacing $a=1.4 \lambda$. When the lattice spacing is larger, interactions become weaker, and cooperative effects are diminished, leading to a narrower distribution of collective mode linewidths.

do not disappear entirely. The radiative decay rates still range from $0.2 \Gamma$ to $3 \Gamma$.

The dramatically narrowed radiative resonance linewidth of some of the collective modes and the sensitive dependence of the narrowing on the spatial separation of the resonators indicates a strong cooperative response of the system to EM fields. For very closely spaced resonators, multiple scattering is considerably influenced by recurrent scattering events in which the field repeatedly scatters from the same meta-atoms. In the example studied here, this leads to the resonance linewidth narrowing of almost five orders of magnitude. Such narrowing could not have been described by independent scatterer approach.

The recurrent scattering that is responsible for the dramatic linewidth narrowing can be characterized by repeated scattering events between pairs of scatterers, triplets of scatterers, etc. ${ }^{20,21,23-25,27}$ In the present work, we have not analyzed the relative contribution of the different processes to the distribution of linewidths. In the case of electric dipole scatterers, the contribution, for instance, of repeated exchanges of a photon between pairs of dipoles to the distribution of resonance linewidths was studied in Ref. 54. A similar calculation could, in principle, be performed in our system, although the interplay between the magnetic and electric dipoles may notably complicate the analysis.

An alternative approach to quantify the contribution of different recurrent scattering processes was performed in Ref. 25. Numerical simulation results were compared with the equations for correlation functions. One, in essence, constructs a hierarchy of equations in which the $n$th level describes the recurrent scattering between subsets of $n$ discrete resonators. Truncating the hierarchy after the $n$th level may, therefore, be used to quantify the contribution of the $n$th order recurrent scattering. In the case of randomly distributed, uncorrelated scatterers, the role of recurrent scattering between $n$ resonators scales with the $n$th power of density. $20,21,23-25,27$ Correlations in the positions of the scatterers modify this density dependence. ${ }^{25,27}$ It was found for the both correlated and uncorrelated samples ${ }^{25}$ that changes in scattering resonance properties as a function of the density of scatterers corresponded to the increased role of recurrent scattering; at higher densities, the higher-order recurrent scattering

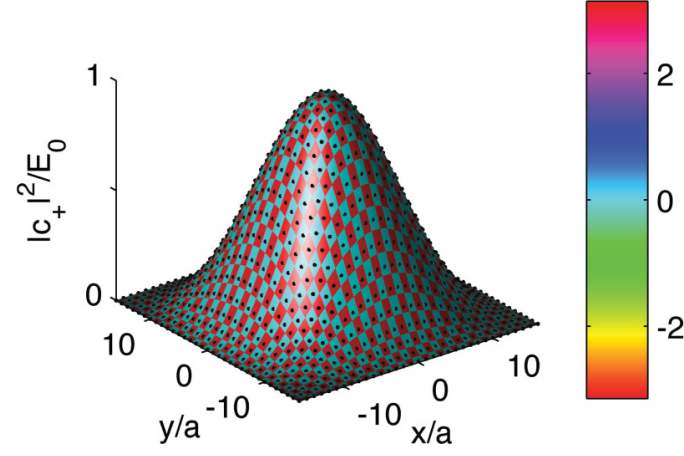

FIG. 3. (Color online) An illustration of the most subradiant of the collective modes in a $33 \times 33$ array of SRRs. The height of the surface represents the energy of the SRR symmetric oscillations $\left|c_{+}\right|^{2}$ normalized to the peak SRR energy $E_{0}=\max _{\ell}\left(\left|c_{+, \ell}\right|^{2}+\left.c_{-, \ell}\right|^{2}\right)$. The colored patches indicate the phase of the electric dipole oscillations for each SRR. The black dots indicate the position of each SRR, while their height indicates the normalized total energy within each unit cell, so that the energy in the magnetic dipole oscillations is given by the difference between the black dots' height and the height of the surface. This subradiant mode is ferroelectric in nature, and has a radiative emission rate $1.2 \times 10^{-5} \Gamma$. The lattice spacing $a=0.5 \lambda$, the meta-atom separation within the SRRs $u=0.12 \lambda$, and $\Gamma_{\mathrm{E}}=\Gamma_{\mathrm{M}}$.

processes become increasingly more important leading to the emergence of more strongly subradiant modes. ${ }^{23-25}$

We now examine the characteristics of some of the collective modes in a $33 \times 33$ lattice with an inter-SRR separation of $a=0.5 \lambda$. As with a single SRR, we can characterize the state of the system by specifying a complex amplitude for both the symmetric (electric) and antisymmetric (magnetic) oscillations. Where the state of the system is fully specified by the vector of single meta-atom amplitudes $\left(b_{1}, b_{2}, \ldots, b_{2 N_{x} N_{y}}\right)^{T}$, we represent the electric and magnetic oscillations of a single SRR, labelled by $\ell=1, \ldots, N_{x} N_{y}$, as $c_{+, \ell}$ and $c_{-, \ell}$, respectively, where

$$
c_{ \pm, \ell}=\frac{1}{\sqrt{2}}\left(b_{2 \ell-1} \pm b_{2 \ell}\right) .
$$

As noted earlier, the subwavelength proximity of adjacent SRRs permits the creation of extremely subradiant collective modes. We illustrate the most subradiant of these modes for a lattice spacing of $a=0.5 \lambda$ in Fig. 3. The energy of this mode resides almost exclusively in symmetric oscillations of the SRRs. However, although the meta-atoms in each SRR oscillate symmetrically, the electric dipole of each unit-cell resonator element points in the opposite direction to that of its nearest neighbor. This mode is antiferroelectric in nature. The phase of each electric dipole, indicated by the color of the unit cell, forms a checkerboard pattern in the phase profile. This mode consists of more strongly excited electric dipole oscillations in the center of the array with smaller contributions from SRRs on the edges. When this mode is excited, the fields emitted from the SRRs tend to remain trapped in the ensemble as they repeatedly scatter from one meta-atom to another. The scattered fields will leak out if this mode very slowly as indicated by the collective emission rate of $1.2 \times 10^{-5} \Gamma$.

The most superradiant of the collective modes, shown in Fig. 4, by contrast couples very strongly to radiation 


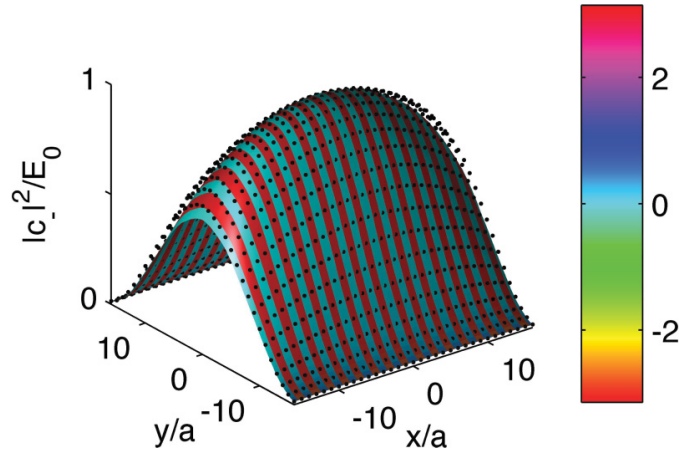

FIG. 4. (Color online) An illustration of the most superradiant of the collective modes in a $33 \times 33$ array of SRRs. The height of the surface represents the energy of the SRR antisymmetric oscillations $\left|c_{-}\right|^{2}$ normalized to the peak SRR energy $E_{0}=\max _{\ell}\left(\left|c_{+, \ell}\right|^{2}+\left.c_{-, \ell}\right|^{2}\right.$. The black dots indicate the position of each SRR, while their height indicates the normalized total energy within each unit cell. The total excitation, $\left|c_{+, \ell}\right|^{2}$, of the electric dipole oscillations is given by the difference between the black dots' height and the height of the surface. All parameters of the ensemble are as in Fig. 3. This superradiant mode has a radiative emission rate of about $11 \Gamma$ and consists largely of magnetic dipole oscillations whose phase variation is matched with EM waves propagating in the $\pm x$ directions.

propagating away from the ensemble. This mode is almost entirely magnetic in nature with the SRRs oscillating antisymmetrically. These magnetic dipole oscillations consist of stripes of constant phase in the $y$ direction, while the phase variation in the $x$ direction is phase matched with radiation propagating along $\pm \hat{\mathbf{e}}_{x}$. An EM plane wave propagating in the $\pm x$ direction whose magnetic field is polarized in the $z$ direction would have an electric field polarized along $\pm \hat{\mathbf{e}}_{y}$. Since the electric dipoles in this most superradiant of modes are largely unexcited, this mode radiates into an equal superposition of EM fields propagating in the positive and negative $x$ directions. The collective excitation coupling to these propagating fields results in a spontaneous emission rate of $11 \Gamma$, more than ten times the single meta-atom emission rate.

In many experimental situations, however, a plane-wave incident field, with nearly uniform phase and intensity in the metamaterial plane, drives the ensemble. The incident field propagates perpendicular to the plane of the metamaterial along the $z$ direction so that it drives the SRRs in phase. It is, therefore, worthwhile to examine modes whose oscillations are phase matched with the incident field since they can be addressed directly. The two modes of interest are the uniform electric mode, with all electric dipoles oscillating in phase, and the uniform magnetic mode, where all magnetic dipoles oscillate in phase.

Figure 5 shows the structure of the uniform electric mode. As desired, an excitation in this mode has its energy almost purely in electric dipole oscillations of the split rings. Furthermore, because all electric dipoles oscillate in phase, this mode efficiently couples to EM fields propagating out of the plane along $\pm \hat{\mathbf{e}}_{z}$ whose electric field polarization is along the electric dipoles $\hat{\mathbf{d}}=\hat{\mathbf{e}}_{y}$. Because the fields scattered by this mode propagate out of the plane, excitation of the mode by an incident plane wave results in reflection of the incident field

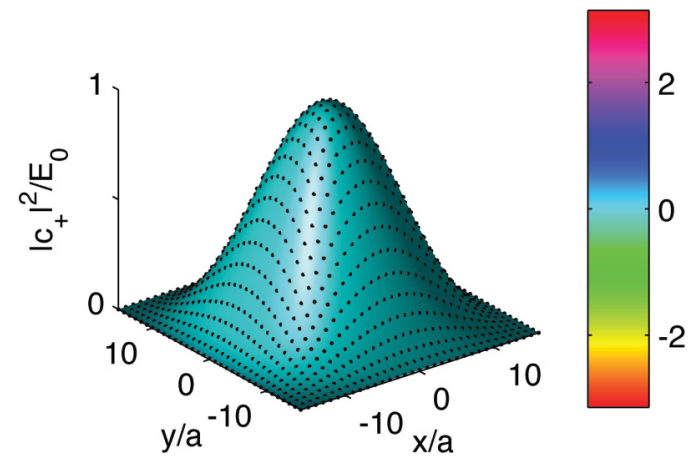

FIG. 5. (Color online) An illustration of the uniform electric collective mode in a $33 \times 33$ array of SRRs. The height of the surface represents the excitation energy of the SRR symmetric oscillations $\left|c_{+}\right|^{2}$ normalized to the peak SRR energy $E_{0}=\max _{\ell}\left(\left|c_{+, \ell}\right|^{2}+\left.c_{-, \ell}\right|^{2}\right.$. The colored patches indicate the phase of the electric dipole oscillations for each SRR. The black dots indicate the position of each SRR, while their height indicates the normalized total energy within each unit cell. All parameters of the ensemble are as in Fig. 3. This mode consists of the split ring electric dipoles oscillating in phase and has a radiative emission rate of approximately the single meta-atom emission rate $\Gamma$.

from the metamaterial. In the geometry considered here, the uniform electric mode has a radiative decay rate of $\gamma_{e} \approx \Gamma$, about as strong as the single meta-atom decay rate.

The second phase matched mode, the uniform magnetic mode, is illustrated in Fig. 6. This uniform mode is almost purely magnetic in nature, with all of the metamolecule magnetic moments oscillating in phase, producing a sheet of magnetization pointing out of the metamaterial. In contrast to the uniform electric mode, however, this mode cannot strongly couple to fields propagating out of the plane. In fact, we have found that for lattice spacings sufficiently less than a wavelength, scattered radiation remains trapped in the ensemble and this mode is subradiant. Here, with a lattice spacing of $a=0.5 \lambda$, the radiative emission rate is suppressed

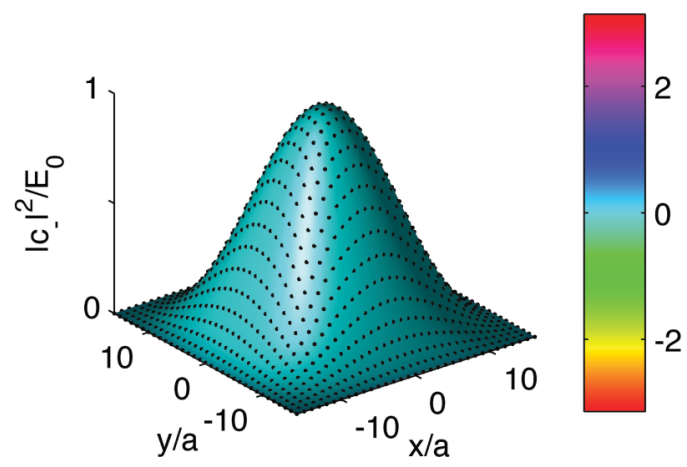

FIG. 6. (Color online) An illustration of the uniform magnetic collective mode in a $33 \times 33$ array of SRRs. The height of the surface represents the energy of the SRR antisymmetric oscillations $\left|c_{-}\right|^{2}$ normalized to the peak SRR energy $E_{0}=\max _{\ell}\left(\left|c_{+, \ell}\right|^{2}+\left.c_{-, \ell}\right|^{2}\right.$. The black dots indicate the position of each SRR, while their height indicates the normalized total energy within each unit cell. All parameters of the ensemble are as in Fig. 3. This mode is composed of all SRRs oscillating antisymmetrically, producing magnetic dipoles in phase. The radiative emission rate of this mode is $0.02 \Gamma$. 
by about a factor of 50 below the single meta-atom decay rate. The form of the magnetic mode does not differ substantially from that in Fig. 6 for larger lattice spacings; however, interresonator spacing affects cooperative interactions and strongly influence the mode's decay rate. ${ }^{46}$ In Ref. 46 , it was shown how a subradiant mode analogous to the uniform magnetic mode we discussed here is responsible for the transmission resonance observed in an array of ASRs. ${ }^{32}$

The calculated collective modes of the system also determine the propagation dynamics of localized excitations. The propagation of excitations are influenced by strong interactions between the resonators. Specifically, in disordered systems, where the locations of scatterers vary randomly, the transition to localization can be characterized from transport properties. ${ }^{37}$ In the studied system, the positions of the resonators are fixed, so the propagation dynamics is determined by the particular excitation. An initial excitation of SSR dipoles will be comprised of some linear combination of collective modes. The more radiant components will quickly decay, leaving behind only the contributions from subradiant modes which oscillate at differing frequencies. This behavior manifests itself as a decaying propagation and spreading of current oscillations through the metamaterial as EM fields scatter in the array. The lifetime of the residual excitation strongly depends on the presence of recurrent scattering and subradiant modes.

In order to demonstrate the time dynamics of excitations, we have studied the specific example of an excitation of the left-most strip of magnetic dipoles along the $y$ axis in the square array. Such a pattern will lose $90 \%$ of its energy and propagate a single lattice site in a time $t=10 / \Gamma$ for a lattice spacing of $a=0.5 \lambda$. As the excitation propagates, it begins to broaden so that at time $t=500 / \Gamma$, the remaining excitation, containing $2 \times 10^{-4}$ of the initial energy, has spread through the sample. When the lattice spacing is larger, $a=1.5 \lambda$, the excitation spreads more quickly through the sample (at time $20 / \Gamma$ ), indicating weaker EM-mediated interactions between the resonators. In this case, only $5 \times 10^{-8}$ of the initial energy has not been radiated away.

\section{QUANTIZING THE METAMATERIAL DYNAMICS}

In this paper, we have developed a general formalism to describe collective oscillations in ensembles of meta-atoms that comprise a metamaterial. In systems where thermal losses are suppressed and can be neglected, however, this formalism can easily be quantized. In the quantized system, the metaatom dynamic variables $Q_{j}$ and their conjugate momenta $\phi_{j}$, whose Poisson brackets are $\left\{Q_{j}, \phi_{j^{\prime}}\right\}=\delta_{j, j^{\prime}}$, become quantum mechanical operators $\hat{Q}_{j}$ and $\hat{\phi}_{j}$ that obey the commutation relations

$$
\begin{aligned}
{\left[\hat{Q}_{j}, \hat{Q}_{j^{\prime}}\right] } & =\left[\hat{\phi}_{j}, \hat{\phi}_{j^{\prime}}\right]=0, \\
{\left[\hat{Q}_{j}, \hat{\phi}_{j^{\prime}}\right] } & =i \hbar \delta_{j, j^{\prime}}
\end{aligned}
$$

When quantizing the system, the classical normal variables undergo the transformations $b_{j} \rightarrow \sqrt{\hbar} \hat{b}_{j}$ and $b_{j}^{*} \rightarrow \sqrt{\hbar} \hat{b}_{j}^{\dagger}$. The normal variables thus become harmonic oscillator creation and annihilation operators that obey the commutation relations

$$
\begin{aligned}
& {\left[\hat{b}_{j}, \hat{b}_{j^{\prime}}\right]=\left[\hat{b}_{j}^{\dagger}, \hat{b}_{h^{\prime}}^{\dagger}\right]=0,} \\
& {\left[\hat{b}_{j}, \hat{b}_{j^{\prime}}^{\dagger}\right]=\delta_{j, j^{\prime}} .}
\end{aligned}
$$

Similarly, the normal variables for the EM field [see Eqs. (23) and (24)] transform as $a_{\mathbf{q}, \lambda} \rightarrow \sqrt{\hbar} \hat{a}_{\mathbf{q}, \lambda}$. The EM field normal variables then commute with those of the meta-atoms and satisfy the commutation relations

$$
\begin{aligned}
& {\left[\hat{a}_{\mathbf{q}, \lambda}, \hat{a}_{\mathbf{q}^{\prime}, \lambda^{\prime}}\right]=\left[\hat{a}_{\mathbf{q}, \lambda}^{\dagger}, \hat{a}_{\mathbf{q}^{\prime}, \lambda^{\prime}}^{\dagger}\right]=0,} \\
& {\left[\hat{a}_{\mathbf{q}, \lambda}, \hat{a}_{\mathbf{q}^{\prime}, \lambda^{\prime}}^{\dagger}\right]=\delta_{\lambda, \lambda^{\prime}} \delta\left(\mathbf{q}-\mathbf{q}^{\prime}\right) .}
\end{aligned}
$$

The ability to easily quantize this formalism may be useful in describing the interactions of low loss metamaterials with nonclassical fields. Furthermore, generalizations of the formalism to nonlinear metamaterials, e.g., involving superconductors, may in and of itself produce nonclassical cooperative effects.

\section{CONCLUSIONS}

In conclusion, we developed a theoretical formalism to describe cooperative interactions of a magnetodielectic metamaterial sample with an EM field. We modeled the metamaterial as an ensemble of discrete EM resonators, or meta-atoms, that each support a single mode of current oscillation. The meta-atoms could, for example, be subwavelength circuit elements that support plasmonic oscillations. From a Lagrangian describing dynamics of the EM field and its interactions with systems of charged particles, we derived the conjugate momenta for the EM field and meta-atom dynamic variables as well as Hamiltonian for the metamaterial system. Hamilton's equations of motion then describe a coupled dynamics between the meta-atoms and the EM field.

We showed how the EM fields are emitted from excited current oscillations within each meta-atom, and in turn, how the EM fields drive the meta-atom dynamics. A single metaatom interacting with its own self-generated field behaves as a radiatively damped LC circuit. In an ensemble of resonators, the meta-atoms also interact with each other. Initially excited by an external field, a meta-atom emits EM radiation that then impinges on other meta-atoms. The other meta-atoms then rescatter the field. Multiple scattering events mediate an interaction between the meta-atoms' current oscillations. The interactions culminate in a discrete, coupled set of equations for the meta-atoms, which describe the collective metamaterial dynamics. The coupled dynamics constituted the main results of this article.

In Sec. V, we examined the collective dynamics in a regime where the influence of a meta-atom's self-generated fields dominates over that of the incident field or the fields scattered by all other meta-atoms in the metamaterial. This assumption allowed us to employ the rotating wave approximation to simplify the description of the dynamics. Appendix C, on the other hand, generalized the formalism to provide for a dynamical description outside the limits of the RWA.

A metamaterial possesses as many collective modes as there are meta-atoms in the sample, each with its own resonance frequency and decay rate. These collective modes can behave very differently from oscillations in a single, 
isolated meta-atom. The cooperative interactions could result in superradiant modes in which energy is radiated away more quickly than an ensemble of meta-atoms acting independently. Other modes, by contrast, are subradiant, for which the mode's radiative emission rate is suppressed. As an example, we examined the dynamics of a planar metamaterial formed from a $33 \times 33$ square lattice of SRRs. When the resonators are closely spaced the collective modes have a broad distribution of radiative decay rates. For a lattice spacing of $0.5 \lambda$, cooperative interactions suppress the most subradiant mode's emission rate by about five orders of magnitude, while the most super-radiant mode radiates eleven times faster than a single meta-molecule. Similarly, by analyzing the propagation dynamics of current excitations in the metamaterial array using the collective eigenmodes, we found that the close spacing of resonators suppresses the rate at which magnetic dipole excitations spread over the sample. In addition to SRRs, the formalism we developed could be used to describe interactions between emitters with other geometries, e.g., dielectric spheres. ${ }^{55}$

The collective dynamics derived from the discrete resonator model can be successfully employed to explain experimentally observed phenomena. For example, in Ref. 46, we used this model to calculate the resonance linewidth narrowing as a function of the system size, associated with the experimental observations of the transmission resonance by Fedotov et al. ${ }^{32}$ The theoretical model provided an excellent agreement with experimental findings. This example illustrates how the formalism developed here lays the ground work allowing one to model collective dynamics in large metamaterial systems in which finite-size effects or irregularities may play a role.

\section{ACKNOWLEDGMENTS}

We would like to thank the EPSRC and the Leverhulme trust for financial support. We also thank N. Papasimakis, V. Fedotov, M. O. Borgh, and N. Zheludev for discussions.

\section{APPENDIX A: THE LAGRANGIAN AND THE POWER-ZIENAU-WOOLLEY TRANSFORMATION}

In this Appendix, we derive the Lagrangian describing the dynamics of meta-atoms interacting with the EM field given in Eq. (13). We start from the standard Lagrangian for the EM field in the Coulomb gauge interacting with arbitrary charge and current distributions. Then, using the Power-Zienau-Woolley transformation, ${ }^{41-43}$ we express the equivalent Lagrangian in terms of polarization and magnetization densities. Given the expressions for the polarization and magnetization densities in Eq. (10), we express the Lagrangian in terms of effective magnetic fluxes and EMFs as in Eq. (13).

An arbitrary vector field $\mathbf{V}(\mathbf{r})$ can be decomposed into its longitudinal $\mathbf{V}_{\|}$and transverse $\mathbf{V}_{\perp}$ components,

$$
\mathbf{V}=\mathbf{V}_{\|}+\mathbf{V}_{\perp}
$$

defined such that $\nabla \times \mathbf{V}_{\|} \equiv 0$ and $\nabla \cdot \mathbf{V}_{\perp} \equiv 0$. In the Coulomb gauge, the EM vector potential is set purely transverse by requiring that $\nabla \cdot \mathbf{A}(\mathbf{r})=0$. It follows from Maxwell's equations that $\mathbf{B}(\mathbf{r})$ is purely transverse and that the longitudinal component of the electric field $\mathbf{E}_{\|}$is not a true dynamical variable but is given by an algebraic relation by the charge density. ${ }^{50}$ In particular, we may write

$$
\mathbf{E}_{\|}=-\nabla U,
$$

where

$$
U(\mathbf{r})=\frac{1}{4 \pi \epsilon_{0}} \int d^{3} r^{\prime} \frac{\rho\left(\mathbf{r}^{\prime}\right)}{\left|\mathbf{r}-\mathbf{r}^{\prime}\right|}
$$

is the scalar potential. The Coulomb energy $V_{\text {Coul }}$ is given in terms of the meta-atom charge densities in Eq. (15) and can be expressed directly in terms of $\mathbf{E}_{\|}$as

$$
V_{\text {Coul }}=\frac{\epsilon_{0}}{2} \int d^{3} r\left|\mathbf{E}_{\|}\right|^{2} .
$$

The transverse component of the electric field is given in terms of the vector potential $\mathbf{A}$ as

$$
\mathbf{E}_{\perp}=-\dot{\mathbf{A}} \text {. }
$$

The standard Lagrangian in the Coulomb gauge may be written as

$$
\mathcal{L}_{\mathrm{C}}=\mathcal{K}-V_{\text {Coul }}+\mathcal{L}_{\mathrm{EM}}+\mathcal{L}_{\mathrm{I}}
$$

where

$$
\mathcal{L}_{\mathrm{I}}=\int d^{3} r \mathbf{j}(\mathbf{r}, t) \cdot \mathbf{A}(\mathbf{r}, t)
$$

accounts for the interaction between the matter and the free EM field, and $\mathbf{j} \equiv \sum_{j} \mathbf{j}_{j}$ is the total current density with the contribution from meta-atom $j$. The meta-atom current densities $\mathbf{j}_{j}$ are given in terms of the generalized velocities $I_{j}=\dot{Q}_{j}$ by Eq. (10). The vector potential $\mathbf{A}(\mathbf{r}, t)$ provides the continuum of dynamic variables describing the evolution of the EM field. The EM field dynamics in the absence of charge and current sources is governed by the Lagrangian, $\mathcal{L}_{\mathrm{EM}}$ [see Eq. (18)]. The charge carriers that give rise to the charge and current densities have an inertia, and hence the current in a meta-atom, resulting from the motion of these carriers, must have an associated kinetic energy. This kinetic energy $\mathcal{K}$ is given in terms of phenomenological inertial inductances in Eq. (14).

The canonical momentum for the fields in the Coulomb gauge is given in terms of the time derivative of the vector potential and is proportional to the transverse component of the electric field:

$$
\Pi^{(\mathrm{C})}(\mathbf{r}) \equiv \frac{\partial \mathcal{L}_{\mathrm{C}}}{\partial \dot{\mathrm{A}}}=\epsilon_{0} \dot{\mathrm{A}}(\mathbf{r})=-\epsilon_{0} \mathbf{E}_{\perp}(\mathbf{r}) .
$$

Similarly, the canonical momentum corresponding to the charges $Q_{j}$ is given by

$$
\phi_{j}^{(\mathrm{C})} \equiv \frac{\partial \mathcal{L}_{C}}{\partial I_{j}}=l_{j} I_{j}+\chi_{j}(t),
$$

where

$$
\chi_{j} \equiv \int d^{3} r \mathbf{A}(\mathbf{r}, t) \cdot\left[\mathbf{p}_{j}(\mathbf{r})+\nabla \times \mathbf{w}_{j}(\mathbf{r})\right] .
$$

The factor $\chi_{j}$ originates from the interaction Lagrangian $\mathcal{L}_{\text {I }}$ [see Eq. (A7)]; its specific form arises from how the current density $\mathbf{j}_{j}$ within each meta-atom $j$ depends on that meta-atom's generalized velocity $I_{j}$ [see Eq. (10b)]. This factor represents an averaged projection of the vector potential onto the current oscillation's mode functions $\mathbf{p}_{j}$ and $\mathbf{w}_{j}$. The 
Hamiltonian in the Coulomb gauge may then be derived from the Lagrangian [see Eq. (A6)]

$$
\mathcal{H}^{(C)}=\frac{1}{2 l_{j}}\left(\phi_{j}-\chi_{j}\right)^{2}+\mathcal{H}_{\mathrm{EM}}^{(C)}+V_{\mathrm{Coul}},
$$

where the energy of the transverse EM field, or the radiation field, is responsible for the excitations of the meta-atoms,

$$
\mathcal{H}_{\mathrm{EM}}^{(\mathrm{C})}=\frac{\epsilon_{0}}{2} \int d^{3} r\left[\mathbf{E}_{\perp}^{2}(\mathbf{r})+c^{2} \mathbf{B}^{2}(\mathbf{r})\right] .
$$

The quantity $\chi_{j}$ originates from the assumption that a mode of current oscillation depends on a single dynamic variable with units of charge. The amplitude of the charge distribution may change in time, but its spatial distribution will not. By contrast, in the more familiar scenario where one describes the motion of particles with fixed charge $q_{j}$ at a time varying position $\mathbf{r}_{j}(t)$, the conjugate momentum for the position coordinates is given by the vector $\dot{\mathbf{r}}_{j}+q_{j} \mathbf{A}\left(\mathbf{r}_{j}(t)\right)$. The scalar quantity $\chi_{j}$ arising from our model plays the same role as the quantity $q_{j} \mathbf{A}\left(\mathbf{r}_{j}(t)\right)$ appearing in the familiar minimal coupling Hamiltonian for moving charged particles.

Although Eq. (A11) is analogous to the standard minimal coupling Hamiltonian description of charged particles in an EM field, it does not turn out to be the most suitable representation to study the interaction of discrete scatterers with the EM field. We find it convenient to express the dynamics in terms of polarization and magnetization densities rather than charge and current densities. In this way, when the circuit elements are much smaller than a wavelength of EM field with which they interact, we may more easily treat the dynamics in terms of interacting electric and magnetic multipoles. To that end, we employ the Power-Zienau-Woolley transformation. ${ }^{43}$ For any globally neutral charge distribution with respective charge and current density $\rho$ and $\mathbf{j}$, there exists a corresponding polarization $\mathbf{P}$ and magnetization density $\mathbf{M}$ such that

$$
\begin{aligned}
\rho(\mathbf{r}, t) & =-\nabla \cdot \mathbf{P}(\mathbf{r}, t), \\
\mathbf{j}(\mathbf{r}, t) & =\dot{\mathbf{P}}(\mathbf{r}, t)+\nabla \times \mathbf{M}(\mathbf{r}, t) .
\end{aligned}
$$

Here, the polarization density is a function of the dynamic variables $Q_{j}$ and the magnetization density is a function of their rates of change $I_{j}$ [see Eq. (9)]. One can modify the Lagrangian by adding the total time derivative $d F / d t$ of a function to the original Lagrangian. Here, we take

$$
F=-\int d^{3} r \mathbf{P}(\mathbf{r}, t) \cdot \mathbf{A}(\mathbf{r}, t),
$$

and the equivalent Lagrangian in the length gauge is thus

$$
\mathcal{L}=\mathcal{L}_{\mathrm{C}}+\frac{d F}{d t} .
$$

Because $F$ is only a function of the dynamic variables $Q_{j}$ and $\mathbf{A}$, the Lagrange equations of motion are invariant under this transformation. Explicitly, adding $d F / d t$ to the interaction term $\mathcal{L}_{I}$ yields

$$
\mathcal{L}_{\mathrm{I}}^{\prime} \equiv \mathcal{L}_{I}+\frac{d F}{d t}=\int d^{3} r(\mathbf{j}-\dot{\mathbf{P}}) \cdot \mathbf{A}-\int d^{3} r \mathbf{P} \cdot \dot{\mathbf{A}} .
$$

From Eq. (A13b), the first integral in Eq. (A16) can be expressed as

$$
\int d^{3} r(\mathbf{j}-\dot{\mathbf{P}}) \cdot \mathbf{A}=\int d^{3} r \mathbf{A} \cdot(\nabla \times \mathbf{M}) .
$$

Integrating this by parts, we obtain the interaction Lagrangian

$$
\mathcal{L}_{\mathrm{I}}^{\prime}=-\int d^{3} r \mathbf{B} \cdot \mathbf{M}-\int d^{3} r \dot{\mathbf{A}} \cdot \mathbf{P} .
$$

To evaluate the second integral, we recognize that $-\dot{\mathbf{A}}=\mathbf{E}+$ $\nabla U$, where $U(\mathbf{r}, t)$ is the electric scalar potential. The last integral appearing in Eq. (A18) thus becomes

$$
-\int d^{3} r \dot{\mathbf{A}} \cdot \mathbf{P}=\int d^{3} r \mathbf{E} \cdot \mathbf{P}+\int d^{3} r \mathbf{P} \cdot \nabla U .
$$

We integrate the last term of Eq. (A19) by parts, and because $U$ is the Coulomb gauge scalar potential, we obtain

$$
\int d^{3} r \mathbf{P} \cdot \nabla U=-\int d^{3} r(\nabla \cdot \mathbf{P}) U=\int d^{3} r \rho U=2 V_{\text {Coul }} .
$$

Therefore the Lagrangian in the Power-Zienau-Woolley picture can be expressed in terms of the total electric and magnetic fields as

$$
\mathcal{L}=\mathcal{K}+V_{\text {Coul }}+\mathcal{L}_{\mathrm{EM}}+\int d^{3} r[\mathbf{B} \cdot \mathbf{M}+\mathbf{E} \cdot \mathbf{P}] .
$$

Although we derived the Lagrangian in Eq. (A21) for a system composed of ensembles of circuit elements, its form is valid for any system of charges where the charge density is described by any generalized dynamic variables and the current density is a function of their generalized velocities. In our system, the total polarization $\mathbf{P}=\sum_{j} \mathbf{P}_{j}$ and magnetization $\mathbf{M}=\sum_{j} \mathbf{M}_{j}$, with the corresponding densities $\mathbf{P}_{j}$ and $\mathbf{M}_{j}$ expressed in terms of the dynamic variable $Q_{j}$ and velocity $I_{j}$ for meta-atom $j$ given by Eqs. (9). Thus, in an ensemble of meta-atoms, the system Lagrangian is given by Eq. (13).

\section{APPENDIX B: ELIMINATION OF INSTANTANEOUS, NONLOCAL INTERACTIONS IN THE POWER-ZIENAU-WOOLLEY PICTURE}

In this Appendix, we provide details of the derivation of the Hamiltonian in the length gauge obtained by the PowerZienau-Woolley transformation. The derivation is analogous to the one discussed in Ref. 50 in determining the Power-ZienauWoolley Hamiltonian for systems of charged particles. We begin by examining the portion of the Hamiltonian, $\mathcal{H}_{\mathrm{E}}$ [see Eq. (30)],

$$
\mathcal{H}_{\mathrm{E}}=\int d^{3} r \mathbf{E}_{\perp} \cdot \mathbf{D}-\int d^{3} r \mathbf{E} \cdot \mathbf{P}-V_{\text {Coul }}-\mathcal{L}_{\mathrm{EM}} .
$$

We will show how the Coulomb potential is absorbed by the Power-Zienau-Woolley Hamiltonian. We will express each term in $\mathcal{H}_{\mathrm{E}}$ in terms of the displacement field $\mathbf{D}$ and the polarization density $\mathbf{P}$. The various components then combine to yield the Hamiltonian for the free EM field, $\mathcal{H}_{\mathrm{EM}}$ [see Eq. (32)], the local polarization contact interaction, and an interaction between the polarization density and the displacement field. 
We noted in Sec. IV A that an advantage of working with the Hamiltonian formalism in the Power-Zienau-Woolley picture is that long-range, instantaneous interactions between meta-atoms do not appear in the Hamiltonian. In any treatment of electrodynamics, the instantaneous noncausal nature of the Coulomb interaction is canceled by other noncausal contributions to dynamics. The form of this cancelation, however, is often rather subtle. In the Power-Zienau-Woolley Hamiltonian, the Coulomb potential is absorbed into a local polarization self-energy. Interactions between meta-atoms are then mediated entirely by the variables describing the scattered EM fields.

In carrying out the simplification, it is useful to note the following properties of the longitudinal and transverse components of any two vector fields $\mathbf{V}_{1}$ and $\mathbf{V}_{2}$. The first is that

$$
\int d^{3} r \mathbf{V}_{1, \|}(\mathbf{r}) \cdot \mathbf{V}_{2, \perp}(\mathbf{r})=0
$$

and, as a consequence,

$$
\int d^{3} r \mathbf{V}_{1} \cdot \mathbf{V}_{2}=\int d^{3} r\left(\mathbf{V}_{1, \|} \cdot \mathbf{V}_{2, \|}+\mathbf{V}_{1, \perp} \cdot \mathbf{V}_{2, \perp}\right)
$$

We also note, that because the charge density in our ensemble of meta-atoms is accounted for entirely by the polarization [see Eq. (A13a)], the displacement field $\mathbf{D}$ is transverse, i.e., $\mathbf{D}=$ $\mathbf{D}_{\perp}$. We may therefore write the transverse and longitudinal electric fields as

$$
\mathbf{E}_{\perp}=\frac{1}{\epsilon_{0}}\left(\mathbf{D}-\mathbf{P}_{\perp}\right), \quad \mathbf{E}_{\|}=-\frac{1}{\epsilon_{0}} \mathbf{P}_{\|} .
$$

The Coulomb interaction energy [see Eq. (A4)] and the Lagrangian for the free electromagnetic field [see Eq. (18)] then becomes

$$
\begin{gathered}
V_{\text {Coul }}=\frac{1}{2 \epsilon_{0}} \int d^{3} r\left|\mathbf{P}_{\|}\right|^{2}, \\
\mathcal{L}_{\mathrm{EM}}=\frac{1}{2 \epsilon_{0}} \int d^{3} r\left(|\mathbf{D}|^{2}-c^{2}|\mathbf{B}|^{2}\right) \\
+\frac{1}{2 \epsilon_{0}} \int d^{3} r\left|\mathbf{P}_{\perp}\right|^{2}-\frac{1}{\epsilon_{0}} \int d^{3} r \mathbf{D} \cdot \mathbf{P} .
\end{gathered}
$$

Similarly, the other two integrals appearing in Eq. (B1) can be expressed as

$$
\begin{gathered}
\int d^{3} r \mathbf{E}_{\perp} \cdot \mathbf{D}=\frac{1}{\epsilon_{0}} \int d^{3} r\left(|\mathbf{D}|^{2}-\mathbf{D} \cdot \mathbf{P}\right), \\
\int d^{3} r \mathbf{E} \cdot \mathbf{P}=\frac{1}{\epsilon_{0}} \int d^{3} r\left(\mathbf{D} \cdot \mathbf{P}-|\mathbf{P}|^{2}\right) .
\end{gathered}
$$

By the property of Eq. (B3), we may write the portion of the Hamiltonian $\mathcal{H}_{\mathrm{E}}$ as

$$
\mathbf{H}_{\mathrm{E}}=\mathcal{H}_{\mathrm{EM}}+\frac{1}{2 \epsilon_{0}} \int d^{3} r|\mathbf{P}|^{2}-\frac{1}{\epsilon_{0}} \int d^{3} r \mathbf{D} \cdot \mathbf{P},
$$

where $\mathcal{H}_{\mathrm{EM}}$, given in Eq. (32), is the Hamiltonian for the electromagnetic field. The second term in Eq. (B9) has absorbed the Coulomb interaction and results only in a local meta-atom self-interaction as discussed in Sec. IV A. The final term of Eq. (B9) accounts for interaction between the distribution of electric dipoles in the polarization density and the displacement field. The total Hamiltonian for the system is then given in Eq. (31).

\section{APPENDIX C: COLLECTIVE INTERACTIONS OF STRONGLY INTERACTING META-ATOMS OUTSIDE THE ROTATING WAVE APPROXIMATION}

In Sec. V, we saw how the EM field scattered from the metamaterial elements produces interactions between metaatom current oscillations in the RWA. For this approximation to be strictly valid, the meta-atoms must weakly interact with the field, radiatively decaying at rates much slower than the oscillator frequencies. In many metamaterial systems, however, such assumptions can be violated, and the RWA may not be employed. In this Appendix, we develop a more general framework for the dynamics that allows us to account for very strong radiative coupling between the oscillator variables. We begin by reframing the equations of motion for the dynamic variables $Q_{j}$ and their conjugate momenta in terms of column vectors of scaled quantities [see Eqs. (135) and (136)]

$$
\begin{gathered}
\tilde{Q} \equiv\left(\tilde{Q}_{1}, \tilde{Q}_{2}, \ldots, \tilde{Q}_{N}\right)^{T}, \\
\tilde{\phi} \equiv\left(\tilde{\phi}_{1}, \ldots, \tilde{\phi}_{N}\right)^{T}
\end{gathered}
$$

in the frequency domain. For each meta-atom $j$, the scaled charge $\tilde{Q}_{j}$ and its scaled conjugate momentum $\tilde{\phi}_{j}$ are slowly varying, with bandwidths comparable to that of the incident field's positive frequency component. In the time domain, they are related to the physical quantities $Q_{j}$ and $\phi_{j}$ by

$$
\begin{aligned}
Q_{j}(t) & =\sqrt{\omega_{j} C_{j}}\left[e^{-i \Omega_{0} t} \tilde{Q}_{j}(t)+e^{i \Omega_{0} t} \tilde{Q}_{j}^{*}(t)\right], \\
\phi_{j}(t) & =\sqrt{\omega_{j} L_{j}}\left[e^{-i \Omega_{0} t} \tilde{\phi}_{j}(t)+e^{i \Omega_{0} t} \tilde{\phi}_{j}^{*}(t)\right] .
\end{aligned}
$$

In deriving the coupling between the elements, we will find that $\tilde{\phi}$ is related to the scaled currents [see Eq. (138)]

$$
\tilde{\mathrm{I}} \equiv\left(\tilde{I}_{1}, \ldots, \tilde{I}_{N}\right)^{T}
$$

through a dimensionless mutual inductance matrix $\mathcal{M}$, and similarly, that the vector of scaled EMFs [see Eq. (116)]

$$
\tilde{\mathcal{E}} \equiv\left(\tilde{\mathcal{E}}_{1}, \ldots, \tilde{\mathcal{E}}_{N}\right)^{T}
$$

is related to $\tilde{Q}$ through a matrix resembling a mutual capacitance. Since the meta-atoms are separated by significant fractions of a wavelength and interactions between them are mediated by the radiated field, $\tilde{\phi}$ contains an additional contribution from $\tilde{Q}$. In addition, $\tilde{\mathcal{E}}$ is linearly coupled to $\tilde{\phi}$. This is because oscillating dipoles, whether electric or magnetic, produce both electric and magnetic fields which drive $\tilde{\mathcal{E}}$ and $\tilde{\phi}$, respectively. We find that, in general, this produces an additional nontrivial coupling between resonators.

We first examine the behavior of the Fourier components of $\tilde{\mathrm{Q}}$ and $\tilde{\phi}$ for a frequency $\Omega>0$, detuned from the central frequency of the incident field by $\delta=\Omega-\Omega_{0}$. Since, by construction, $\tilde{\mathrm{Q}}, \tilde{\phi}, \tilde{\mathcal{E}}$, and $\tilde{\Phi}$ are related only to the positive frequency components of $Q_{j}, \phi_{j}, \mathcal{E}_{j}$, and $\Phi_{j}$, the scaled variables have no Fourier components for $\delta<-\Omega_{0}$. From the equations of motion for the unscaled variables [see Eq. (38)] and the definitions of the scaled variables, we arrive at the 
relations for $\delta>-\Omega_{0}$ :

$$
\begin{aligned}
& -i\left(\delta+\Omega_{0}\right) \tilde{\mathrm{Q}}(\delta)=\omega \tilde{\mathrm{I}}(\delta), \\
& -i\left(\delta+\Omega_{0}\right) \tilde{\phi}(\delta)=\tilde{\mathcal{E}}(\delta),
\end{aligned}
$$

where $\omega$ represents a diagonal matrix whose elements $[\omega]_{j, j} \equiv$ $\omega_{j}$ are the resonance frequencies of the individual meta-atoms. Equations (C7) represent the coupling of the meta-atom dynamic variables to the EM fields, including the incident field, the fields emitted by all other meta-atoms, and the field generated from the meta-atom itself. The self-interactions were derived in Sec. V A [see Eqs. (94) and (95)], while we obtained the contributions from the scattered fields in Sec. VB [see Eqs. (144) and (145) with $\delta+\Omega_{0}$ substituted for $\Omega_{0}$ ].

As presently written, Eq. (C7a) states in terms of scaled variables in frequency space that the rate of change of the meta-atom charge is equal to its current. Here, we are interested in how these rates of change are related to the states of the meta-atom dynamic variables and their conjugate momenta. To express these currents $\tilde{I}$ in terms of charges and conjugate momenta, we recognize that the conjugate momentum is the sum of the magnetic flux and the current multiplied by the kinetic inductance $l_{j}$ [see Eq. (19)],

$$
\tilde{\phi}_{j}=\frac{l_{j}}{L_{j}} \tilde{I}_{j}+\tilde{\Phi}_{j} .
$$

The scaled fluxes, $\tilde{\Phi}_{j}$ [see Eq. (117)], contain contributions from the meta-atoms' self-generated fields [see Eq. (95)], which result in magnetic self-inductances as well as to the magnetic fields generated externally. The kinetic and magnetic self-inductances combine to provide the total self-inductance [see Eq. (98)]. Equation (139) thus relates the conjugate momentum, $\tilde{\phi}_{j}$, for meta-atom $j$ to its current $\tilde{I}_{j}$ and the externally generated flux. The contributions to the external flux from the incident magnetic field and fields scattered from other meta-atoms in the system [see Eq. (145) with $\Omega$ substituted for $\Omega_{0}$ ] combine to provide the external magnetic driving of individual meta-atoms. We synthesize these contributions to obtain the relationship between the column vectors of scaled conjugate momenta $\tilde{\phi}$, currents $\tilde{\mathrm{I}}$, charges $\tilde{Q}$, and fluxes induced by the incident field $\tilde{\Phi}_{\text {in }}$ expressed as

$$
\tilde{\phi}(\delta)=\mathcal{M}(\Omega) \tilde{\mathrm{I}}(\delta)-\omega^{-1} \Upsilon_{\mathrm{M}}^{\frac{1}{2}} \mathcal{G}_{\times}^{T}(\Omega) \Upsilon_{\mathrm{E}}^{\frac{1}{2}} \tilde{\mathrm{Q}}(\delta)+\tilde{\Phi}_{\text {in }}(\delta),
$$

where we have redefined the diagonal matrices $\Upsilon_{\mathrm{E}}$ and $\Upsilon_{M}$ containing the meta-atom electric and magnetic dipole emission rates so that they reflect the frequency dependence of meta-atom scattering rates outside the RWA. These matrices have the diagonal matrix elements

$$
\begin{aligned}
{\left[\Upsilon_{\mathrm{E}}\right]_{j, j} } & \equiv\left(\frac{\Omega}{\omega_{j}}\right)^{3} \Gamma_{\mathrm{E}, j}, \\
{\left[\Upsilon_{\mathrm{M}}\right]_{j, j} } & \equiv\left(\frac{\Omega}{\omega_{j}}\right)^{3} \Gamma_{\mathrm{E}, j} .
\end{aligned}
$$

The scaled mutual inductance $\mathcal{M}$, is given by

$$
\mathcal{M}(\Omega)=\left(1+i \omega^{-1} \Upsilon_{\mathrm{M}}+\omega^{-1} \Upsilon_{\mathrm{M}}^{\frac{1}{2}} \mathcal{G}_{\mathrm{M}} \Upsilon_{\mathrm{M}}^{\frac{1}{2}}\right),
$$

and the matrices $\mathcal{G}_{\mathrm{M}}$ and $\mathcal{G}_{\times}$are given in Eq. (146). The diagonal portion of $\mathcal{M}$ has both real and imaginary components: the real part is the self-inductances' contribution to this scaled mutual inductance matrix, while the imaginary part arises from emission of magnetic dipole radiation from the meta-atoms current oscillations. Solving Eq. (C9) for I, yields

$$
\tilde{\mathrm{I}}(\delta)=\mathcal{M}^{-1}(\Omega)\left[\tilde{\phi}(\delta)-\tilde{\Phi}_{\mathrm{in}}(\delta)+\omega^{-1} \Upsilon_{\mathrm{M}}^{\frac{1}{2}} \mathcal{G}_{\times}^{T}(\Omega) \Upsilon_{\mathrm{E}}^{\frac{1}{2}} \tilde{\mathrm{Q}}(\delta)\right] .
$$

The current $\tilde{I}_{j}$ on an element $j$ is not just related to the conjugate momentum $\tilde{\phi}_{j}$, but to the conjugate momenta and charges of all other meta-atoms in the system, as well as the flux from the incident field. In the absence of electric dipole radiation $\Upsilon_{\mathrm{E}}=0$, we recover the relationship between currents and magnetic field fluxes found in systems of interacting, radiating, inductive circuits. The additional coupling that results from oscillating electric dipoles when $\Upsilon_{\mathrm{E}} \neq 0$ adds some richness to the dynamics of metamaterial systems outside the RWA.

As with the magnetic fluxes, the EMFs $\tilde{\mathcal{E}}$ contain contributions from the self-generated electric fields of the meta-atom [see Eq. (94)] and from electric fields generated by all other meta-atoms in the system [see Eq. (144) with $\Omega$ substituted for $\Omega_{0}$ ]. From the previous results, we can express the column vector of EMFs as

$$
\begin{aligned}
\tilde{\mathcal{E}}(\delta)= & -\left[\omega-i \Upsilon_{\mathrm{E}}-\Upsilon_{\mathrm{E}}^{\frac{1}{2}} \mathcal{G}_{\mathrm{E}}(\Omega) \Upsilon_{\mathrm{E}}^{\frac{1}{2}}\right] \tilde{\mathrm{Q}}(\delta) \\
& +\Upsilon_{\mathrm{E}}^{\frac{1}{2}} \mathcal{G}_{\times}(\Omega) \Upsilon_{\mathrm{M}}^{\frac{1}{2}} \tilde{\mathrm{I}}(\delta)+\tilde{\mathcal{E}}_{\mathrm{in}}(\delta) .
\end{aligned}
$$

The diagonal matrix, $\omega-i \Upsilon_{\mathrm{E}}$, results from the coupling of each element with its self-generated field where $\Upsilon_{E}$ accounts for decay due to electric dipole radiation. The matrix $\Upsilon_{\mathrm{E}}^{\frac{1}{2}} \mathcal{G}_{\mathrm{E}}(\Omega) \Upsilon_{\mathrm{E}}^{\frac{1}{2}}$, where $\mathcal{G}_{\mathrm{E}}$ is given in Eq. (146), provides dipole-dipole coupling between electric polarization densities of distinct meta-atoms, while $\Upsilon_{\mathrm{E}}^{\frac{1}{2}} \mathcal{G}_{\times}(\Omega) \Upsilon_{\mathrm{M}}^{\frac{1}{2}}$ provides radiated contributions of oscillating magnetic dipoles to the EMFs. Since we wish to express the EMFs exclusively in terms of the charges and their conjugate momenta, we eliminate I by substituting Eq. (C13) into Eq. (C14) to obtain

$$
\begin{aligned}
\tilde{\mathcal{E}}(\delta)= & -\omega \Xi^{-1}(\Omega) \tilde{\mathrm{Q}}(\delta)+\Upsilon_{\mathrm{E}}^{\frac{1}{2}} \mathcal{G}_{\times}(\Omega) \Upsilon_{\mathrm{M}}^{\frac{1}{2}} \mathcal{M}^{-1}(\Omega) \tilde{\phi}(\delta) \\
& +\tilde{\mathcal{E}}_{\text {in }}(\delta)-\Upsilon_{\mathrm{E}}^{\frac{1}{2}} \mathcal{G}_{\times}(\Omega) \Upsilon_{\mathrm{M}}^{\frac{1}{2}} \mathcal{M}^{-1}(\Omega) \tilde{\Phi}_{\text {in }}(\delta),
\end{aligned}
$$

where $\Xi(\Omega)$ is an effective dimensionless mutual capacitance matrix defined such that

$$
\begin{aligned}
\Xi^{-1}(\Omega) \equiv & 1-i \omega^{-1} \Upsilon_{\mathrm{E}}-\omega^{-1} \Upsilon_{\mathrm{E}}^{\frac{1}{2}} \mathcal{G}_{\mathrm{E}}(\Omega) \Upsilon_{\mathrm{E}}^{\frac{1}{2}} \\
& -\omega^{-1} \Upsilon_{\mathrm{E}}^{\frac{1}{2}} \mathcal{G}_{\times}(\Omega) \Upsilon_{\mathrm{M}}^{\frac{1}{2}} \mathcal{M}^{-1}(\Omega) \omega^{-1} \Upsilon_{\mathrm{M}}^{\frac{1}{2}} \mathcal{G}_{\times}^{T}(\Omega) \Upsilon_{\mathrm{E}}^{\frac{1}{2}},
\end{aligned}
$$

where the matrix expression on the final line arises from the expression for current in terms of conjugate momenta and charges. This matrix expression is reminiscent of a scattering process in which oscillating charges couple to oscillating conjugate momenta in other meta-atoms via $\Upsilon_{M}^{\frac{1}{2}} \mathcal{G}_{\times}^{T}(\Omega) \Upsilon_{\mathrm{E}}^{\frac{1}{2}}$, these conjugate momenta are transformed into currents by $\mathcal{M}^{-1}(\Omega)$, and these currents produce electric fields in neighboring meta-atoms through $\Upsilon_{\mathrm{E}}^{\frac{1}{2}} \mathcal{G}_{\times}(\Omega) \Upsilon_{\mathrm{M}}^{\frac{1}{2}}$. 
Having expressed the currents [see Eq. (C13)] and EMFs [see Eq. (C15)] exclusively in terms of charges, conjugate momenta and driving due to the incident field, we may finally write the equations of motion in frequency space for the slowly varying charges $\tilde{Q}(\delta)$ and conjugate momenta $\tilde{\phi}(\delta)$ :

$$
\begin{aligned}
-i(\delta & \left.+\Omega_{0}\right)\left(\begin{array}{c}
\tilde{\mathrm{Q}}(\delta) \\
\tilde{\phi}(\delta)
\end{array}\right) \\
= & \left(\begin{array}{cc}
\omega \mathcal{M}^{-1} \omega^{-1} \Upsilon_{\mathrm{M}}^{\frac{1}{2}} \mathcal{G}_{\times}^{T} \Upsilon_{\mathrm{E}}^{\frac{1}{2}} & \omega \mathcal{M}^{-1} \\
-\omega \Xi^{-1} & \Upsilon_{\mathrm{E}}^{\frac{1}{2}} \mathcal{G}_{\times} \Upsilon_{M}^{\frac{1}{2}} \mathcal{M}^{-1}
\end{array}\right) \\
& \times\left(\begin{array}{c}
\tilde{\mathrm{Q}}(\delta) \\
\tilde{\phi}(\delta)
\end{array}\right)+\left(\begin{array}{c}
-\omega \mathcal{M}^{-1} \tilde{\Phi}_{\text {in }}(\delta) \\
\tilde{\mathcal{E}}_{\mathrm{in}}(\delta)-\Upsilon_{\mathrm{E}}^{\frac{1}{2}} \mathcal{G}_{\times} \Upsilon_{\mathrm{M}}^{\frac{1}{2}} \mathcal{M}^{-1} \tilde{\Phi}_{\mathrm{in}}(\delta)
\end{array}\right) .
\end{aligned}
$$

The physical content of Eq. (C17) becomes more evident in the limits where the meta-atoms are spaced far enough apart that their interactions can be seen as interactions between point sources [i.e., where the approximation in Eq. (147) holds] and when the magnetic interaction is weak $\left(\Gamma_{\mathrm{M}, j} \ll \Omega_{0}\right)$. Then, writing Eq. (C17) to lowest order in $\Gamma_{\mathrm{M}, j} / \omega_{j}$, we obtain dynamic equations where $\tilde{Q}_{j}$ and $\tilde{\phi}_{j}$ are driven by fields scattered from the meta-atom electric dipoles $\mathbf{d}_{j^{\prime}}^{(+)}(\Omega) \equiv h_{j^{\prime}} \sqrt{\omega_{j^{\prime}} C_{j^{\prime}}} \tilde{Q}_{j^{\prime}}(\delta)$ and magnetic dipoles $\mathbf{m}_{j^{\prime}}^{(+)}(\Omega) \equiv A_{j^{\prime}} \sqrt{\omega_{j^{\prime}} / L_{j^{\prime}}} \tilde{I}_{j^{\prime}}(\delta)$ [see Eqs. (77), (135), (136), (138), and (147)]:

$$
\begin{aligned}
- & i \Omega \tilde{Q}_{j}(\delta)+\left[\omega_{j}-i\left(\frac{\Omega}{\omega_{j}}\right)^{3} \Gamma_{\mathrm{M}, j}\right] \tilde{\phi}_{j}(\delta) \\
= & -\omega_{j} \tilde{\Phi}_{\mathrm{in}, j}(\delta)-\alpha_{j} \hat{\mathbf{m}}_{j} \cdot \frac{\mu_{0} k^{3}}{4 \pi} \sum_{j^{\prime} \neq j}\left[\mathbf{G}\left(\mathbf{r}_{j}-\mathbf{r}_{j^{\prime}}, \Omega\right)\right. \\
& \left.\cdot \mathbf{m}_{j^{\prime}}^{(+)}(\Omega)-c \mathbf{G}_{\times}\left(\mathbf{r}_{j}-\mathbf{r}_{j^{\prime}}, \Omega\right) \cdot \mathbf{d}_{j^{\prime}}^{(+)}(\Omega)\right], \quad(\mathrm{C} 18 a) \\
- & i \Omega \tilde{\phi}_{j}(\delta)+\left[\omega_{j}-i\left(\frac{\Omega}{\omega_{j}}\right)^{3} \Gamma_{\mathrm{E}, j}\right] \tilde{Q}_{j}(\delta) \\
= & \tilde{\mathcal{E}}_{\mathrm{in}, j}+\chi_{j} \hat{\mathbf{d}}_{j} \cdot \frac{k^{3}}{4 \pi \epsilon_{0}} \sum_{j^{\prime} \neq j}\left[\mathbf{G}\left(\mathbf{r}_{j}-\mathbf{r}_{j^{\prime}}, \Omega\right) \cdot \mathbf{d}_{j^{\prime}}^{(+)}(\Omega)\right. \\
& \left.+\frac{1}{c} \mathbf{G}_{\times}\left(\mathbf{r}_{j}-\mathbf{r}_{j^{\prime}}, \Omega\right) \cdot \mathbf{m}_{j^{\prime}}^{(+)}(\Omega)\right],
\end{aligned}
$$

where $\alpha_{j} \equiv\left(2 A_{j} / 3\right) \sqrt{\omega_{j} / L_{j}} / 3$ and $\chi_{j} \equiv 2 h_{j} \sqrt{\omega_{j} C_{j}} / 3$. The incident field drives the meta-atom electric and magnetic dipoles, producing the terms $-\omega_{j} \tilde{\Phi}_{\mathrm{in}, j}$ and $\tilde{\mathcal{E}}_{\mathrm{in}, j}$. The effects of the meta-atoms' self-generated fields are included on the left-hand side of Eq. (C18). The magnetic fields produced by all other meta-atomic dipoles in the system $j^{\prime} \neq j$ drive the dynamics of $\tilde{Q}_{j}$, while the electric fields scattered from these meta-atoms drive the dynamics of the conjugate momentum $\tilde{\phi}_{j}$.

In principle, one could solve Eq. (C17) in the narrow bandwidth approximation in which the driving field envelopes $\tilde{\mathbf{E}}_{\text {in }}$ and $\tilde{\mathbf{B}}_{\text {in }}$ vary on time scales much larger than $1 / \Omega_{0}$. One would accomplish this by substituting $\Omega_{0}$ for $\Omega$ in the coupling matrices $\mathcal{G}_{\mathrm{E} / \mathrm{M} / \times}$ and $\Upsilon_{\mathrm{E} / \mathrm{M}}$, then inverse Fourier transforming Eq. (C17). This procedure, however, may not be particularly illuminating. We find it useful to explore the dynamics in terms of the oscillator normal variables b. But first, we will revisit the basic characteristics of these normal variables to understand how they behave outside the RWA.

\section{The normal meta-atom variables revisited}

In Sec. V, we have assumed the meta-atom normal variables $\mathrm{b}=\left(b_{1}, b_{2}, \ldots, b_{N}\right)^{T}$ are slowly varying and are proportional to the slowly varying envelopes of the charges $\tilde{Q}$ and conjugate momenta $\tilde{\phi}$. This was a good approximation when we assumed each meta-atom coupled to its self-generated fields much more strongly than it couples to the external fields, i.e., when $\Gamma_{\mathrm{E}, j}, \Gamma_{\mathrm{M}, j} \ll \omega_{j}, \Omega_{0}$. In those limits, the external field interactions act as a perturbation that slowly alters the dominant behavior of the meta-atoms oscillating as effective LC circuits. When the coupling to the external field is stronger, however, we will see that this is no longer the case.

While the normal variables are slowly varying in the RWA, in general, they contain fast oscillating components even when $\tilde{Q}$ and $\tilde{\phi}$ have narrow bandwidths. To see this, we rewrite the normal variables [defined in Eq. (109)] in terms of the slowly varying dynamic variables. Recall that in terms of the slowly varying quantities, the original charges and conjugate momenta are expressed as

$$
\begin{aligned}
& \frac{Q_{j}(t)}{\sqrt{\omega_{j} C_{j}}}=e^{-i \Omega_{0} t} \tilde{Q}_{j}(t)+e^{i \Omega_{0} t} \tilde{Q}_{j}^{*}(t), \\
& \frac{\phi_{j}(t)}{\sqrt{\omega_{j} L_{j}}}=e^{-i \Omega_{0} t} \tilde{\phi}_{j}(t)+e^{i \Omega_{0} t} \tilde{\phi}_{j}^{*}(t) .
\end{aligned}
$$

We therefore express the column vector of normal variables as

$$
\mathrm{b}(t)=\tilde{\mathrm{b}}^{(+)}(t)+e^{2 i \Omega_{0}}\left[\tilde{\mathrm{b}}^{(-)}(t)\right]^{*},
$$

where we have defined the slowly varying normal variable components

$$
\tilde{\mathrm{b}}^{( \pm)}(t) \equiv \frac{1}{\sqrt{2}}[\tilde{\mathrm{Q}}(t) \pm i \tilde{\phi}(t)]
$$

The normal variables $b$ therefore have a bimodal spectrum with a slowly varying component peaked at zero frequency and fast oscillating component peaked at frequency $-2 \Omega_{0}$. Note that despite the apparent similarity between the definition of $b_{j}$ [see Eq. (109)] and $\tilde{\mathrm{b}}^{( \pm)}$[see Eq. $(\mathrm{C} 21)$ ], $\tilde{\mathrm{b}}^{(-)}$is not equal to $\left[\tilde{b}^{(+)}\right]^{*}$. In the RWA, for example, we ignore the rapidly rotating term $\left\{\exp \left(2 i \Omega_{0}\right)\left[\tilde{\mathrm{b}}^{(-)}(t)\right]^{*}\right.$ in Eq. $\left.(\mathrm{C} 20)\right\}$ and make the approximation $\tilde{b}^{(-)} \approx 0$. Physically, the RWA implies that the normalized conjugate momentum $\tilde{\phi}_{j}(t)$ has the same amplitude as $\tilde{Q}_{j}(t)$ but its oscillation lags by a definite phase $\pi / 2$ in accordance with Eq. (137). However, outside the RWA, the contributions of $\tilde{b}^{(-)}$cannot be neglected.

\section{Normal variable dynamics outside the RWA}

We can obtain the normal variable dynamics from those for the slowly varying charges and conjugate momenta from Eq. (C17). In terms of the normal variables the vectors Q $\tilde{Q}$ and $\tilde{\phi}$ are given by

$$
\left(\begin{array}{l}
\tilde{\mathrm{Q}} \\
\tilde{\phi}
\end{array}\right)=\frac{1}{\sqrt{2}}\left(\begin{array}{cc}
1 & 1 \\
-i & i
\end{array}\right)\left(\begin{array}{l}
\tilde{\mathrm{b}}^{(+)} \\
\tilde{\mathrm{b}}^{(-)}
\end{array}\right) .
$$


The vectors of normal variables $\tilde{b}^{( \pm)}$therefore evolve according to

$$
\begin{aligned}
-i \delta\left(\begin{array}{c}
\tilde{\mathrm{b}}^{(+)}(\delta) \\
\tilde{\mathrm{b}}^{(-)}(\delta)
\end{array}\right)= & \left(\begin{array}{ll}
\mathcal{C}^{(+,+)}(\Omega) & \mathcal{C}^{(+,-)}(\Omega) \\
\mathcal{C}^{(-,+)}(\Omega) & \mathcal{C}^{(-,-)}(\Omega)
\end{array}\right)\left(\begin{array}{l}
\tilde{\mathrm{b}}^{(+)}(\delta) \\
\tilde{\mathrm{b}}^{(-)}(\delta)
\end{array}\right) \\
& +\left(\begin{array}{c}
\mathrm{f}_{\mathrm{in}}^{(+)}(\delta) \\
\mathrm{f}_{\mathrm{in}}^{(-)}(\delta)
\end{array}\right),
\end{aligned}
$$

where $\mathcal{C}^{\left(s_{1}, s_{2}\right)}\left(s_{1}, s_{2}= \pm\right)$ are $N \times N$ matrices that provide a linear coupling between the column vectors $\tilde{\mathrm{b}}^{\left(s_{2}\right)}$ and $\tilde{\mathrm{b}}^{\left(s_{1}\right)}$. These coupling matrices are given by

$$
\begin{aligned}
\mathcal{C}^{\left(s_{1}, s_{2}\right)} \equiv & i\left(\Omega_{0}-s_{1} \omega\right) \delta_{s_{1}, s_{2}}-\frac{s_{1} \Upsilon_{\mathrm{E}}+s_{2} \Upsilon_{\mathrm{M}} \mathcal{M}^{-1}}{2} \\
& +i \frac{s_{1} \Upsilon_{\mathrm{E}}^{\frac{1}{2}} \mathcal{G}_{\mathrm{E}} \Upsilon_{\mathrm{E}}^{\frac{1}{2}}+s_{2} \Upsilon_{\mathrm{M}}^{\frac{1}{2}} \mathcal{G}_{\mathrm{M}} \Upsilon_{\mathrm{M}}^{\frac{1}{2}} \mathcal{M}^{-1}}{2} \\
& +\frac{1}{2}\left(\omega+i s_{1} \Upsilon_{\mathrm{E}}^{\frac{1}{2}} \mathcal{G}_{\times} \Upsilon_{\mathrm{M}}^{\frac{1}{2}}\right) \mathcal{M}^{-1} \omega^{-1} \Upsilon_{\mathrm{M}}^{\frac{1}{2}} \mathcal{G}_{\times}^{T} \Upsilon_{\mathrm{E}}^{\frac{1}{2}} \\
& +\frac{s_{1} s_{2} \Upsilon_{\mathrm{E}}^{\frac{1}{2}} \mathcal{G}_{\times} \Upsilon_{\mathrm{M}}^{\frac{1}{2}} \mathcal{M}^{-1}}{2} .
\end{aligned}
$$

The top line of Eq. (C24) contains the diagonal elements of the matrices $\mathcal{C}^{\left(s_{1}, s_{2}\right)}$ that arise from the interaction of the meta-atoms with their self-generated fields. For example the diagonal elements of $\mathcal{C}^{(+,+)}$contain the detunings $\Delta$ [see Eq. (152)] and the total radiative decay rate $\Upsilon_{\mathrm{E}}+\Upsilon_{\mathrm{M}}$. The interaction matrices $\mathcal{C}^{\left(s_{1}, s_{2}\right)}$ contain the effects of all scattering processes, including those resulting from scattered electric fields emitted from electric and magnetic dipoles and those resulting from magnetic fields emitted from magnetic and electric dipoles. Interaction with the incident field produces the driving represented by the column vectors

$$
\mathrm{f}_{\mathrm{in}}^{( \pm)}(\delta) \equiv \frac{1}{\sqrt{2}}\left[ \pm i \tilde{\mathcal{E}}-\left(\omega \pm i \Upsilon_{\mathrm{E}}^{\frac{1}{2}} \mathcal{G}_{\times} \Upsilon_{\mathrm{M}}^{\frac{1}{2}}\right) \mathcal{M}^{-1} \tilde{\Phi}_{\text {in }}\right]
$$

The EM mediated interactions simplify greatly if we assume the self-inductance of a meta-atom is much greater than the mutual inductance between any two meta-atoms. A necessary condition for this is that $\Gamma_{\mathrm{M}, j} \ll \omega_{j}$ for all $j$ since $\mathcal{M}=1+O\left(\Gamma_{\mathrm{M}} \omega^{-1}\right)$. In this limit, we neglect all contributions of order $\Gamma_{\mathrm{M}, j} / \omega_{j}$ to the mutual inductance, allowing us to make the substitution $\mathcal{M}^{-1} \approx 1$. This yields

$$
\begin{aligned}
\mathcal{C}^{\left(s_{1}, s_{2}\right)} \approx & i\left(\Omega_{0}-s_{1} \omega\right) \delta_{s_{1}, s_{2}}-\frac{s_{1} \Upsilon_{\mathrm{E}}+s_{2} \Upsilon_{\mathrm{M}}}{2} \\
+ & i \frac{s_{1} \Upsilon_{\mathrm{E}}^{\frac{1}{2}} \mathcal{G}_{\mathrm{E}} \Upsilon_{\mathrm{E}}^{\frac{1}{2}}+s_{2} \Upsilon_{\mathrm{M}}^{\frac{1}{2}} \mathcal{G}_{\mathrm{M}} \Upsilon_{\mathrm{M}}^{\frac{1}{2}}}{2} \\
+ & \frac{\Upsilon_{\mathrm{M}}^{\frac{1}{2}} \mathcal{G}_{\times}^{T} \Upsilon_{\mathrm{E}}^{\frac{1}{2}}+s_{1} s_{2} \Upsilon_{\mathrm{E}}^{\frac{1}{2}} \mathcal{G}_{\times} \Upsilon_{\mathrm{M}}^{\frac{1}{2}}}{2} .
\end{aligned}
$$

Under the additional assumption that all meta-atom resonance frequencies lie in a narrow bandwidth around $\Omega_{0}$, the matrix providing the dynamic coupling between the various $\tilde{b}_{j}^{(+)}$ and $\tilde{b}_{j^{\prime}}^{(+)}$is identical to the coupling matrix between normal variables in the RWA [see Eq. $(151)$ ], i.e., $\mathcal{C}^{(+,+)} \approx \mathcal{C}$.

\section{Temporal dynamics and collective modes outside the RWA}

When the incident field possesses a narrow bandwidth around $\Omega_{0}$ and varies much more slowly than the time it takes for light to propagate across the metamaterial sample, we can obtain a simple expression for the collective temporal evolution of the metamaterial. With this slowly varying incident field, we can approximate the dynamics by replacing the frequencies $\Omega \equiv \Omega_{0}+\delta$ appearing in the interaction matrices with $\Omega_{0}$. We then inverse Fourier transform Eq. (C23) to obtain

$$
\begin{aligned}
\frac{d}{d t}\left(\begin{array}{c}
\tilde{\mathrm{b}}^{(+)}(t) \\
\tilde{\mathrm{b}}^{(-)}(t)
\end{array}\right)= & \left(\begin{array}{ll}
\mathcal{C}^{(+,+)}\left(\Omega_{0}\right) & \mathcal{C}^{(+,-)}\left(\Omega_{0}\right) \\
\mathcal{C}^{(-,+)}\left(\Omega_{0}\right) & \mathcal{C}^{(-,-)}\left(\Omega_{0}\right)
\end{array}\right)\left(\begin{array}{c}
\tilde{\mathrm{b}}^{(+)}(t) \\
\tilde{\mathrm{b}}^{(-)}(t)
\end{array}\right) \\
& +\left(\begin{array}{c}
\mathrm{f}_{\mathrm{in}}^{(+)}(t) \\
\mathrm{f}_{\mathrm{in}}^{(-)}(t)
\end{array}\right) .
\end{aligned}
$$

These equations describe the collective response of a metamaterial to a narrow bandwidth incident field where the interresonator interactions and emission rates can be arbitrarily large. Unlike the simplified collective dynamics derived in Sec. VB, Eq. (C27) is not subject to the constraints of the RWA.

\section{Recovering the dynamics of the RWA}

The dynamics in the RWA that we explored earlier in Sec. V amounted to neglecting the fast oscillating components of the normal variables b. Here, this equates to assuming $\tilde{b}^{(-)}=0$, and therefore $b=\tilde{b}^{(+)}$. We argued earlier that this approximation is valid in the limits of weak interactioni.e., $\Gamma_{\mathrm{E}, j}, \Gamma_{\mathrm{M}, j} \ll \Omega_{0}$ - and in which all single meta-atom resonance frequencies lie within a narrow bandwidth about the driving frequency, i.e., $\left|\Delta_{j}\right| \ll \Omega_{0}$. Indeed, when the interactions are sufficiently weak, the diagonal elements of $\mathcal{C}^{(-,-)}$[see Eq. $\left.(\mathrm{C} 23)\right]\left[i\left(\Omega_{0}+\omega\right)+\left(\Gamma_{\mathrm{E}}+\Gamma_{\mathrm{M}}\right) / 2\right]$ dominate over every other element of the coupling. As a result, in the response of the metamaterial to the incident field, the elements of $\tilde{b}^{(-)}$would be negligible in comparison to $\tilde{b}^{(+)}$. Note, however, that although $\Gamma_{\mathrm{E} / \mathrm{M}} \ll \Omega_{0}$ is a necessary condition for the validity of the RWA, it is not sufficient in and of itself. This is because the interaction between elements can still become very strong if the separation between them is much less than a wavelength. Here, however, we will assume the inter-element separation is sufficiently large that the limits on $\Gamma_{\mathrm{E} / \mathrm{M}}$ are sufficient. In the RWA, we may therefore expand $\mathcal{C}^{(+,+)}$to lowest order in $\Gamma_{\mathrm{E}}$ and $\Gamma_{\mathrm{M}}$ and make the approximation $\left(\Omega / \omega_{j}\right)^{3} \approx 1$. Upon doing this, we recover precisely the dynamics given in Eq. (149) of Sec. V B.
${ }^{1}$ R. A. Shelby, D. R. Smith, and S. Schultz, Science 292, 77 (2001).

${ }^{2}$ D. R. Smith, W. J. Padilla, D. C. Vier, S. C. Nemat-Nasser, and S. Schultz, Phys. Rev. Lett. 84, 4184 (2000).
${ }^{3}$ D. R. Smith, J. B. Pendry, and M. C. K. Wiltshire, Science 305, 788 (2004).

${ }^{4}$ G. Dolling, C. Enkrich, M. Wegener, C. M. Soukoulis, and S. Lindin, Science 312, 892 (2006). 
${ }^{5}$ J. B. Pendry, Phys. Rev. Lett. 85, 3966 (2000).

${ }^{6}$ V. G. Veselago, Sov. Phys. Usp. 10, 509 (1968).

${ }^{7}$ J. B. Pendry, D. Schurig, and D. R. Smith, Science 312, 1780 (2006).

${ }^{8}$ U. Leonhardt, Science 312, 1777 (2006).

${ }^{9}$ D. Schurig, J. J. Mock, B. J. Justice, S. A. Cummer, J. B. Pendry, A. F. Starr, and D. R. Smith, Science 314, 977 (2006).

${ }^{10}$ D. R. Smith, S. Schultz, P. Marks, and C. M. Soukoulis, Phys. Rev. B 65, 195104 (2002).

${ }^{11}$ T. Koschny, M. Kafesaki, E. N. Economou, and C. M. Soukoulis, Phys. Rev. Lett. 93, 107402 (2004).

${ }^{12}$ J. B. Pendry, A. J. Holden, D. J. Robbins, and W. J. Stewart, IEEE Trans. Microwave Theory Tech. 47, 2075 (1999).

${ }^{13}$ P. A. Belov and C. R. Simovski, Phys. Rev. E 72, 026615 (2005).

${ }^{14}$ R. Liu, T. J. Cui, D. Huang, B. Zhao, and D. R. Smith, Phys. Rev. E 76, 026606 (2007).

${ }^{15}$ C. R. Simovski, Metamaterials 1, 62 (2007).

${ }^{16}$ Z. Li, K. Aydin, and E. Ozbay, Phys. Rev. E 79, 026610 (2009).

${ }^{17}$ D. R. Smith, Phys. Rev. E 81, 036605 (2010).

${ }^{18}$ D. R. Smith and J. B. Pendry, J. Opt. Soc. Am. B 23, 391 (2006).

${ }^{19}$ C. Fietz and G. Shvets, Phys. Rev. B 82, 205128 (2010).

${ }^{20}$ B. A. van Tiggelen, A. Lagendijk, and A. Tip, J. Phys.: Condens. Matter 2, 7653 (1990).

${ }^{21}$ O. Morice, Y. Castin, and J. Dalibard, Phys. Rev. A 51, 3896 (1995).

${ }^{22}$ A. Ishimaru, Wave Propagation and Scattering in Random Media: Multiple Scattering, Turbulence, Rough Surfaces, and RemoteSensing, Vol. 2 (Academic Press, St. Louis, Missouri, 1978).

${ }^{23}$ J. Ruostekoski and J. Javanainen, Phys. Rev. A 55, 513 (1997).

${ }^{24}$ J. Ruostekoski and J. Javanainen, Phys. Rev. A 56, 2056 (1997).

${ }^{25}$ J. Javanainen, J. Ruostekoski, B. Vestergaard, and M. R. Francis, Phys. Rev. A 59, 649 (1999).

${ }^{26}$ P. de Vries, D. V. van Coevorden, and A. Lagendijk, Rev. Mod. Phys. 70, 447 (1998).

${ }^{27}$ J. Ruostekoski and J. Javanainen, Phys. Rev. Lett. 82, 4741 (1999).

${ }^{28}$ C. A. Müller, T. Jonckheere, C. Miniatura, and D. Delande, Phys. Rev. A 64, 053804 (2001).

${ }^{29}$ F. A. Pinheiro, M. Rusek, A. Orlowski, and B. A. van Tiggelen, Phys. Rev. E 69, 026605 (2004).

${ }^{30}$ S. D. Jenkins and J. Ruostekoski, arXiv:1112.6136.

${ }^{31}$ J. Kästel, M. Fleischhauer, and G. Juzeliūnas, Phys. Rev. A 76, 062509 (2007).

${ }^{32}$ V. A. Fedotov, N. Papasimakis, E. Plum, A. Bitzer, M. Walther, P. Kuo, D. P. Tsai, and N. I. Zheludev, Phys. Rev. Lett. 104, 223901 (2010).
${ }^{33}$ Z. Szabó, G.-H. Park, R. Hedge, and E.-P. Li, IEEE Trans. Microwave Theory Tech. 58, 2646 (2010).

${ }^{34}$ N. Papasimakis, V. A. Fedotov, Y. H. Fu, D. P. Tsai, and N. I. Zheludev, Phys. Rev. B 80, 041102(R) (2009).

${ }^{35}$ S. Savo, N. Papasimakis, and N. I. Zheludev, Phys. Rev. B 85, 121104(R) (2012).

${ }^{36}$ D. S. Wiersma, P. Bartolini, A. Lagendijk, and R. Righini, Nature (London) 390, 671 (1997).

${ }^{37}$ B. van Tiggelen, in Diffuse Waves in Complex Media, edited by J. P. Fouque, NATO Advanced Science Institutes Series, Series C: Mathematical and Physical Sciences (Springer, Dordrecht, Netherlands, 1999), Vol. 531, pp. 1-60.

${ }^{38}$ A. Sentenac and P. C. Chaumet, Phys. Rev. Lett. 101, 013901 (2008).

${ }^{39}$ T. S. Kao, S. D. Jenkins, J. Ruostekoski, and N. I. Zheludev, Phys. Rev. Lett. 106, 085501 (2011).

${ }^{40}$ F. Lemoult, G. Lerosey, J. de Rosny, and M. Fink, Phys. Rev. Lett. 104, 203901 (2010).

${ }^{41}$ E. A. Power and S. Zienau, Philos. Trans. R. Soc. 251, 427 (1959).

${ }^{42}$ E. A. Power, Introductory Quantum Electrodynamics, Mathematical Physics Series (Longmans, London, 1964), Vol. 24.

${ }^{43}$ E. A. Power and T. Thirunamachandran, Proc. R. Soc. London A 372, 265 (1980).

${ }^{44}$ H. Liu, D. A. Genov, D. M. Wu, Y. M. Liu, Z. W. Liu, C. Sun, S. N. Zhu, and X. Zhang, Phys. Rev. B 76, 073101 (2007).

${ }^{45}$ H. Liu, D. A. Genov, D. M. Wu, Y. M. Liu, J. M. Steele, C. Sun, S. N. Zhu, and X. Zhang, Phys. Rev. Lett. 97, 243902 (2006).

${ }^{46}$ S. D. Jenkins and J. Ruostekoski, arXiv:1106.5754.

${ }^{47}$ J. D. Jackson, Classical Electrodynamics (Wiley, New York, 1998).

${ }^{48}$ E. Prodan, C. Radloff, N. J. Halas, and P. Nordlander, Science 302, 419 (2003).

${ }^{49}$ H. Wang, D. W. Brandl, P. Nordlander, and N. J. Halas, Acc. Chem. Res. 40, 53 (2006).

${ }^{50}$ C. Cohen-Tannaudji, J. Dupont-Roc, and G. Grynberg, Photons and Atoms: Introduction to Quantum Electrodynamics (Wiley, New York, 1989).

${ }^{51}$ H. Goldstein, Classical Mechanics, 2nd ed. (Addison Wesley, Reading, 1980).

${ }^{52}$ V. A. Fedotov, M. Rose, S. L. Prosvirnin, N. Papasimakis, and N. I. Zheludev, Phys. Rev. Lett. 99, 147401 (2007).

${ }^{53}$ N. Papasimakis (unpublished).

${ }^{54}$ A. Goetschy and S. E. Skipetrov, Phys. Rev. E 84, 011150 (2011).

${ }^{55}$ D. J. Bergman and D. Stroud, Phys. Rev. B 22, 3527 (1980). 